\title{
Boundary Integral Methods for Multicomponent Fluids and Multiphase Materials
}

\author{
T.Y. Hou * J.S. Lowengrub ${ }^{\dagger} \quad$ M.J. Shelley ${ }^{\ddagger}$
}

\begin{abstract}
In this paper, we present a brief review of the application of boundary integral methods in two dimensions to multi-component fluid flows and multi-phase problems in materials science. We focus on the recent development and outcome of methods which accurately and efficiently include surface tension. In fluid flows, we examine the effects of surface tension on the Kelvin-Helmholtz and Rayleigh-Taylor instabilities in inviscid fluids, the generation of capillary waves on the free surface, and problems in Hele-Shaw flows involving pattern formation through the SaffmanTaylor instability, pattern selection, and singularity formation. In materials science, we discuss microstructure evolution in diffusional phase transformations, and the effects of the competition between surface and elastic energies on microstructure morphology. A common link between these different physical phenomena is the utility of an analysis of the appropriate equations of motion at small spatial scales to develop accurate and efficient time-stepping methods.
\end{abstract}

\section{Introduction}

The last 15 years have seen the rapid development of numerical methods, especially in two dimensions, for applying boundary integral methods to multi-fluid problems in fluid dynamics, and more recently to multi-phase problems in materials science. By multi-fluid or multi-phase we mean systems where the constituitive properties of the fluid or material change abruptly at a dividing interface; The case of immiscible fluids, such as oil and water, stands as the classical example. An important and complicating property of such systems is surface tension (or surface energy in the materials context). Much recent effort in the application of boundary integral methods has focused on developing numerical methods that efficiently and accurately include surface tension. And while boundary integral methods are applicable only to a restricted type of flow problem, these problems are central in fluids and materials science. In fluids, these problems include those producing prototype patterns, the first nonlinear stages of immiscible fluids mixing, the development of finite-time singularities, and capillary wave generation in water waves. In materials science, these problems include morphology selection in phase transition dynamics and many-precipitate coarsening, under various types of material anisotropy.

In this paper, we present a review of recent applications of boundary integral methods to simulate interfacial dynamics of multi-component fluids and multi-phase materials with surface tension in two dimensions. A boundary integral representation applies when, for example, the PDEs

\footnotetext{
${ }^{*}$ Dept. of Applied Mathematics, California Institute of Technology, Pasadena, CA 91125

${ }^{\dagger}$ School of Mathematics, University of Minnesota, Minneapolis, MN 55455; Department of Mathematics, University of North Carolina, NC 27599.

${ }^{\ddagger}$ Courant Institute of Mathematical Sciences, New York University, New York, NY 10012.
} 
governing the bulk fluid or material are piecewise homogeneous, and for which a Green's function can be found or approximated. In such cases, the dynamics of the system can be reduced to the self-contained, nonlocal dynamics of the interface separating the homogeneous fluids or phases. In fluid dynamics this typically means that we are dealing with potential flows (e.g. inviscid and irrotational flows, Hele-Shaw flows) or Stokes flows. (Except in special cases we neglect the latter, as Stokes flows are the subject of a separate review in this volume.) Boundary integral methods are not (immediately) applicable to more general interfacial fluid flows, such as those governed by the viscous Navier-Stokes equations. In the materials science context, we focus on diffusional phase transformations whose formulation is closely related to that of Hele-Shaw flows.

For a few specific problems in these areas, we present a historical perspective and then discuss what we believe to be the state-of-the-art in numerical simulation. Because of the limited scope of our review, we refer the reader to more general reviews of interfacial fluid flows by Schwarz \& Fenton [170], Yeung [215], Hyman [88], Romate [158], Prosperetti \& Oguz [145], Stone [180], Hou [85] Sarpkaya [166], Tsai and Yue [198] and Scardovelli \& Zaleski [167]. For diffusional phase transformations in materials science, we refer the reader to the more general reviews by Purdy [150], Voorhees [201, 202] and Johnson \& Voorhees [92].

In addition, it is important to note that there are other, more general, numerical approaches for simulating free boundary problems in fluids and materials. These include level-set, volume-offluid, immersed boundary, front-tracking, phase-field, and discrete atom methods. Several of these approaches are the subject of separate reviews in this volume, and here we focus exclusively on boundary integral methods. When applicable, boundary integral methods outperform these other methodologies in accurately and efficiently capturing the dynamics. And so, while being applicable to a core set of problems in fluid dynamics and materials, boundary integral methods provide excellent bench-mark simulations for comparing these different computational strategies.

There are several difficulties in including the effects of surface tension in a simulation. First, as the pressure jump due to surface tension at an interface is proportional to the interfacial curvature, a high number of spatial derivatives are introduced into the dynamics. This results in high-order constraints on explicit time-stepping methods. Secondly, seemingly natural choices of frame in which to compute the interfacial motion can make these constraints strongly time-dependent, and prohibitive. And thirdly, due to the divergence free condition on the fluid velocity, these curvature dependent terms enter the dynamics nonlocally and nonlinearly. Such difficulties are not specific to the inclusion of surface tension, but also arise when dealing with the dynamics of surfaces or curves that have elastic or other curvature-dependent responses.

Within the context of boundary integral methods for 2-d potential and Hele-Shaw flows, we show in HLS94 [81] how these difficulties arising from surface tension can be subverted, and efficient and accurate numerical methods constructed. This relies in part on the "Small-Scale Decomposition" (SSD), a mathematical analysis which identifies the source of stiffness by examining the equations of motion at small length-scales. The SSD analysis shows that when the equations of motion are properly formulated, surface tension acts through a linear operator at small length-scales. This contribution can then be treated implicitly and efficiently in a time-integration scheme, and the high-order constraints removed. The consequent improvements in efficiency and results can be dramatic. For example, in HLS94 we simulated the very long-time development of densely branched patterns in radial Hele-Shaw flow, and suggested the formation of "topological singularities" in the Kelvin-Helmholtz problem with surface tension. This latter study was continued in HLS97 [82], where we developed nonuniform grid methods, used high-order time-stepping, and quantified many aspects of this singularity through careful numerical simulation. Here we will review many other related efforts and works.

These analytical approaches might point the way to the development of similar methods in more 
complicated situations. For example, simulations of heart function using the immersed boundary method are presently constrained in time-step by the stiffness induced by "fiber" elasticity, which is a curvature dependent boundary force (C. Peskin, private communication). In this situation, one must also consider the rotational and viscous aspects of the fluid flow, set in a very complicated geometry.

In Section 2, we discuss the application of boundary integral methods to inviscid and incompressible multi-fluid flows with surface tension. The prototype problem is the nonlinear development of the Kelvin-Helmholtz under surface tension. We discuss extensions to the Rayleigh-Taylor instability and water waves. In Section 3, we discuss the application of boundary integral methods to Hele-Shaw flows, to study pattern formation and morphology selection, and singularity formation. In Section 4, we discuss the application of boundary integral methods to diffusional phase transitions in materials science. Section 5 gives concluding remarks, and discusses future directions in the application of boundary integral methods.

\section{Inviscid Interfacial Fluid Flows with Surface Tension}

In this section, we present a brief review of recent applications of boundary integral methods to study inviscid, incompressible interfacial flows with surface tension in two dimensions. In particular, we focus on the nonlinear evolution of vortex sheets separating two immiscible fluids and the dynamic generation of capillary waves on a free surface.

Many physically interesting fluid flows involve the motion of interfaces separating immiscible flow components with small viscosity. In flows where there is rapid motion, the effects of viscosity may be secondary in importance to those of surface tension. This is particularly evident in shear flows [196]. Moreover, surface tension is central to understanding fluid dynamic phenomena such as droplet formation and capillary wave motion.

Surface tension at an interface separating two immiscible fluids arises due to an imbalance of the fluid components' intermolecular cohesive forces. It is modeled through the Laplace-Young condition which relates the pressure jump across an interface to the interfacial curvature. As mentioned in the Introduction, the accurate simulation of interfaces with surface tension is a problem of considerable difficulty, and stable, efficient and accurate boundary integral methods have been developed only recently. We review work that we consider to be representative of the current state-of-the-art research in this field.

\subsection{Historical Perspective}

The use of boundary integral methods in inviscid interfacial flows in 2 dimensions has a long and rich history that dates back to the 1932 study of vortex sheet roll-up by Rosenhead [160]. Much later, Birkhoff [26] developed a boundary integral formulation for more general interfacial motion. In 1976, Longuet-Higgins \& Cokelet [120] developed the first successful boundary integral method to compute plunging breakers. Since then, many boundary integral methods have been developed to simulate free-surface Euler flows. See $[5,27,18,21,22,19,13,14,15,16,17,11,33,34,43,53$, $54,64,81,82,104,103,105,132,134,147,151,155,156,172,199,197,200,49,209,214,215,220]$ for a small sample. For a more complete set of references, see the review articles listed in the introduction and the references therein. While it is not our goal to review all of this work here, we point out that the origins of many modern boundary integral algorithms can be traced to the seminal paper of Baker, Meiron \& Orszag (BMO82) [14]. In that paper, a detailed derivation of the boundary integral equations is given and the use of iteration methods, to solve the resulting 
integral equations, is pioneered. BMO82 then applied the methods to study breaking waves over finite-bottom topographies and interacting free-surface waves.

The study of interfacial flows with surface tension in 2 dimensions using boundary integral methods began with the work of Zalosh [218] in which the nonlinear evolution of a vortex sheet was considered (density-matched components). Subsequently, other methods have been developed, for flows with different density flow components, by many others including Rottman \& Olfe [161], Pullin [149], Rangel \& Sirignano [151], Baker \& Moore [15], Kudela [105], Yang [214], Boulton-Stone \& Blake [27], Robinson \& Boulton-Stone [156], and Tulin [199].

All of the boundary integral methods listed above exhibit numerical instability that requires some type of ad-hoc numerical smoothing to yield smooth evolution. The primary difficulty with using smoothing is that it can lead to unphysical results since the effects of smoothing may dominate those of surface tension. In independent works, Beale, Hou \& Lowengrub [18, 22, 19] and Baker \& Nachbin (BN98) [11] identified certain incompatibilities in the spatial discretization of the boundary integral equations, both with and without surface tension. These incompatibilities were shown to lead to numerical instability of the type observed in previous studies. Beale et al. and BN98 presented alternative, highly accurate and stable methods.

Additional difficulties occur when the equations are discretized in time. The differential clustering of interface grid points may result in prohibitive time step restrictions for stability for explicit time integration methods because of the high order derivative terms introduced by surface tension. Because the surface tension appears in the equations nonlocally and nonlinearly, standard implicit time stepping methods are very expensive. To overcome these difficulties, Hou, Lowengrub \& Shelley (HLS94, HLS97) [81, 82] derived an alternate formulation of the equations which has all the nice properties for time integration schemes that are associated with having a linear highest order term (such as viscosity in the Navier-Stokes equations). For example, the methods given in HLS94 and HLS97 are explicit in Fourier space and do not have the severe time step restrictions usually associated with surface tension. The methods are then used to study the nonlinear, long-time evolution of vortex sheets with surface tension, interfaces between fluid components with small density differences (Boussinesq approximation) and Hele-Shaw interfaces.

Later, Ceniceros \& Hou (CH98) [33] proved convergence of a semi-discrete (time continuous) version of the methods proposed in HLS94/HLS97 for general two fluid interfacial flows. CH98 also carefully investigated the effects of surface tension on the Rayleigh-Taylor instability. In addition, Ceniceros \& Hou (CH99a) [34] applied the methods of HLS94/HLS97 to study capillary waves on free surfaces.

Although it is beyond the scope of our review, the use of boundary integral methods in axisymmetric and 3-d interfacial flows is also a very active research area. See, for example, $[10,159,121,123,158,139,28,198,76,136,138,37,76,84,83,20]$. We note that very recently, Nie [135] has extended the methods of HLS94/HLS97 to study the nonlinear evolution of axisymmetric vortex sheets with surface tension.

\subsection{Boundary Integral Formulation}

Consider two inviscid, incompressible, and irrotational fluids separated by the parametrized planar interface $\Gamma$ given by $\mathbf{X}(\alpha)=(x(\alpha), y(\alpha))$, as shown schematically in Fig. 1. The lower fluid is denoted 1 , and the upper fluid is denoted 2. $\hat{\mathbf{n}}$ and $\hat{\mathbf{s}}$ are respectively the unit normal and tangent vectors to $\Gamma$, while $\kappa$ is its curvature. For simplicity, the density is assumed to be constant on each side of $\Gamma$. Here, the velocity on either side of $\Gamma$ is evolved by the incompressible Euler equations

$$
\mathbf{u}_{j t}+\left(\mathbf{u}_{j} \cdot \nabla\right) \mathbf{u}_{j}=-\frac{1}{\rho_{j}} \nabla\left(p_{j}+\rho_{j} g y\right), \quad \nabla \cdot \mathbf{u}_{j}=0
$$


where the subscript $j$ denotes the upper or lower fluid. There are the boundary conditions

(i). $\quad[\mathbf{u}]_{\Gamma} \cdot \hat{\mathbf{n}}=0$, the kinematic boundary condition

(ii). $\quad[p]_{\Gamma}=\tau \kappa$, the dynamic boundary condition

(iii). $\quad \mathbf{u}_{j}(x, y) \rightarrow\left( \pm V_{\infty}, 0\right)$ as $y \rightarrow \pm \infty$, the far - field boundary condition

Here, [.] denotes the jump taken from above to below $\Gamma$. The tangential component of fluid velocity is typically discontinuous at $\Gamma$. Such an interface is called a vortex sheet (see [164]). The velocity at a point $\mathbf{X}$ away from the interface has the integral representation

$$
\mathbf{u}(\mathbf{X})=\frac{1}{2 \pi} \int \gamma\left(\alpha^{\prime}\right) \frac{\left(\mathbf{X}-\mathbf{X}\left(\alpha^{\prime}\right)\right)^{\perp}}{\left|\mathbf{X}-\mathbf{X}\left(\alpha^{\prime}\right)\right|^{2}} d \alpha^{\prime}
$$

where $\mathbf{X}^{\perp}=(-y, x) . \gamma$ is called the (unnormalized) vortex sheet strength. It gives the velocity difference across $\Gamma$ by

$$
\tilde{\gamma}=\frac{\gamma(\alpha)}{s_{\alpha}}=-\left.[\mathbf{u}]\right|_{\Gamma} \cdot \hat{\mathbf{s}}
$$

where $s_{\alpha}=\sqrt{x_{\alpha}^{2}+y_{\alpha}^{2}}$ is the arclength metric. The velocity jump $\tilde{\gamma}$ is called the true vortex sheet strength. This representation is well known; see [14]. We will consider flows that are 1-periodic in the $x$-direction. The average value, $\bar{\gamma}$, of $\gamma$ over a period in $\alpha$ satisfies $-\bar{\gamma} / 2=V_{\infty}$.

While there is a discontinuity in the tangential component of the velocity at $\Gamma$, the normal component, $U(\alpha)$, is continuous and is given by (5) as

$$
\begin{aligned}
U(\alpha) & =\mathbf{W}(\alpha) \cdot \hat{\mathbf{n}} \quad \text { where } \\
\mathbf{W}(\alpha) & =\frac{1}{2 \pi} P . V . \int \gamma\left(\alpha^{\prime}\right) \frac{\left(\mathbf{X}(\alpha)-\mathbf{X}\left(\alpha^{\prime}\right)\right)^{\perp}}{\left|\mathbf{X}(\alpha)-\mathbf{X}\left(\alpha^{\prime}\right)\right|^{2}} d \alpha^{\prime},
\end{aligned}
$$

and P.V. denotes the principal value integral. This integral is called the Birkhoff-Rott integral.

Using the representation (5) of the velocity, Euler's equation at the interface, and the LaplaceYoung condition, the equations of motion for the interface are

$$
\begin{aligned}
\mathbf{X}_{t} & =U \hat{\mathbf{n}}+T \hat{\mathbf{s}} \\
\gamma_{t} & -\partial_{\alpha}\left((T-\mathbf{W} \cdot \hat{\mathbf{s}}) \gamma / s_{\alpha}\right) \\
& =-2 A_{\rho}\left(s_{\alpha} \mathbf{W}_{t} \cdot \hat{\mathbf{s}}+\frac{1}{8} \partial_{\alpha}\left(\gamma / s_{\alpha}\right)^{2}-(T-\mathbf{W} \cdot \hat{\mathbf{s}}) \mathbf{W}_{\alpha} \cdot \hat{\mathbf{s}} / s_{\alpha}\right)-F r^{-1} y_{\alpha}+W e^{-1} \kappa_{\alpha} .
\end{aligned}
$$

Here, the equations have been non-dimensionalized on a periodicity length $\lambda$ and the velocity scale $\bar{\gamma}$, and

$$
\begin{aligned}
& A_{\rho}=\frac{\Delta \rho}{2 \bar{\rho}} \quad \text { is the Atwood ratio }, \\
& F r=\frac{\bar{\rho} \bar{\gamma}^{2} \lambda^{2}}{g(\Delta \rho) \lambda^{3}} \quad \text { is the Froude number, and } \\
& W e=\frac{\bar{\rho} \lambda^{2} \bar{\gamma}^{2}}{\tau \lambda} \quad \text { is the Weber number, }
\end{aligned}
$$

where $\Delta \rho=\rho_{1}-\rho_{2}$, and $\bar{\rho}=\left(\rho_{1}+\rho_{2}\right) / 2$. The Froude number measures the relative importance of inertial forces to gravitational forces, while the Weber number measures the relative importance of inertial forces to the dispersive forces of surface tension forces. 
$T$ is an (as yet) arbitrary tangential velocity that specifies the motion of the parametrization of $\Gamma$. The so-called Lagrangian formulation corresponds to choosing the tangential velocity of a point on the interface to be the arithmetic average of the tangential components of the fluid velocity on either side. That is, choosing $T=\mathbf{W} \cdot \hat{\mathbf{s}}$, in which case Eq. (10) simplifies considerably.

Eq. (10) is a Fredholm integral of the second kind for $\gamma_{t}$ due to the presence of $\gamma_{t}$ in $\mathbf{W}_{t}$. This equation has a unique solution, and is contractive [14]. The mean of $\gamma$ is preserved by Eq. (10) and must be chosen to be $-2 V_{\infty}$, initially, to guarantee that condition (iii) is satisfied. Further, while $\gamma$ is evolved as an independent variable, it cannot be interpreted independently of the parametrization. From Eq. (6), it is the ratio $\tilde{\gamma}=\gamma / s_{\alpha}$ that has a physical interpretation, and $s_{\alpha}$ is determined by the choice of $T$.

Eqs. (9-10) realize different physical situations in different limits of the nondimensional parameters. For example, taking $A_{\rho}=-1$ gives the classical Rayleigh-Taylor problem regularized by surface tension. Taking $A_{\rho}=+1$ gives water waves with surface tension. Taking $A_{\rho}=F r^{-1}=0$ gives the Kelvin-Helmholtz problem of two density matched, immiscible liquids. All of these problems will be considered in the following subsections.

\subsection{The Sources of Stiffness and the Small-Scale Decomposition}

For the Kelvin-Helmholtz problem, a natural choice of frame is the so-called "Lagrangian" frame in which $T=\mathbf{W} \cdot \mathbf{s}$. That is, the interface moves with the average of the velocities on each side. The problem is then completely characterized by the Weber number $W e$, and the Lagrangian formulation of the equations of motion becomes simply

$$
\begin{aligned}
\mathbf{X}_{t}(\alpha, t) & =\mathbf{W}(\alpha, t) \\
\gamma_{t}(\alpha, t) & =W e^{-1} \kappa_{\alpha} .
\end{aligned}
$$

It is this compact formulation that has been employed in various studies of singularity formation in the dynamics of vortex sheets without surface tension (see, for example [131, 104, 128, 172, 42]). Without surface tension, the curvature of the sheet diverges at a finite time and is coupled to a concentration of interfacial vorticity. This is known as the "Moore" singularity after Moore [131].

Next, we demonstrate that the Lagrangian formulation results leads to extreme differential clustering of computational points during typical simulations. This results in severe numerical time step constraints when surface tension is present. This may be seen through a general linear analysis given by Beale, Hou \& Lowengrub [18, 19]. Linearizing around the time dependent inertial vortex sheet $\Gamma=(x(\alpha, t), y(\alpha, t))$ with strength $\gamma(\alpha, t)$, Beale et al. find the leading order equation for the normal component of a perturbation at large wavenumber:

$$
\eta_{t t}=-\frac{\gamma^{2}}{4 s_{\alpha}^{4}} \eta_{\alpha \alpha}+\frac{W e^{-1}}{2 s_{\alpha}^{3}} \mathcal{H}\left[\eta_{\alpha \alpha \alpha}\right] .
$$

Here, $\mathcal{H}$ is the Hilbert transform:

$$
\mathcal{H}[f](\alpha)=\frac{1}{\pi} P . V . \int_{-\infty}^{+\infty} \frac{f\left(\alpha^{\prime}\right)}{\alpha-\alpha^{\prime}} d \alpha^{\prime}
$$

Setting $W e^{-1}=0$ gives the linearly ill-posed behavior of the unregularized Kelvin-Helmholtz problem. For finite $W e$, this ill-posedness is regularized by a dispersion due to surface tension.

A "frozen coefficient" analysis of Eq. (16) reveals that the least restrictive time-dependent stability constraint on a stable explicit time integration method is

$$
\Delta t<C W e^{1 / 2} \cdot\left(\bar{s}_{\alpha} h\right)^{3 / 2}, \text { where } \bar{s}_{\alpha}=\min _{\alpha} s_{\alpha},
$$


see $[18,19]$ for details. Here $h=1 / N$ is the grid spacing, with $N$ the number of points describing $\Gamma$. Since arclength spacing, $\Delta s$, satisfies $\Delta s \approx s_{\alpha} h$, Eq. (17) implies that the stability constraint is in fact determined by the minimum spacing in arclength between adjacent points on the grid.

For several "typical" simulations (same initial data, differing Weber numbers), the evolution of $\bar{s}_{\alpha}$ associated with the Lagrangian formulation is shown in Fig. 2, on a base-ten logarithmic scale. Over the times shown, $\bar{s}_{\alpha}$ decreases in value by a factor of $10^{4}$ or more. Consequently, the time-step constraint (17) decreases by at least a factor of $10^{6}$, even for a fixed spatial grid size $h$. The steep drop at slightly less than $t=0.5$ is the result of the compression associated with the shadow of the Moore singularity, which occurs at $t_{M} \approx 0.37$ for this initial data [104]. Such strongly timedependent time-step constraints have severely limited previous numerical investigations [151, 149, $11]$.

Once a stable spatial discretization has been obtained, the primary challenge to computing the long time evolution of interfacial flows with surface tension lies in the construction of time integration methods with good stability properties. It is difficult to straightforwardly construct efficient implicit time integration methods as the source of the stiffness, the $\kappa_{\alpha}$ in the $\gamma$-equation, involves both a nonlinear combination of high derivatives of the interface position and contributes nonlocally to the motion through the $\gamma$ in the Birkhoff-Rott integral. The approach we consider to be state-of-the-art in generating such time integration methods was first given in HLS94. It involves reformulating the equations of motion according to the following three steps: (A) $\theta-s_{\alpha}$ formulation; (B) small scale analysis; (C) special choices of reference frames (tangential velocities).

\section{(A). $\theta-s_{\alpha}$ Formulation}

Rather than using $x, y$ as the dynamical variables, repose the evolution in variables that are more naturally related to curvature. Motivated by the identity $\theta_{s}=\kappa$, where $\theta$ is the tangent angle to the curve $\Gamma$, the evolution is formulated with $\theta$ and $s_{\alpha}$ as the independent dynamical variables. The equations of motion are then given by

$$
\begin{aligned}
s_{\alpha t} & =T_{\alpha}-\theta_{\alpha} U \\
\theta_{t} & =\frac{1}{s_{\alpha}} U_{\alpha}+\frac{T}{s_{\alpha}} \theta_{\alpha} . \\
\gamma_{t} & =W e^{-1} \partial_{\alpha}\left(\theta_{\alpha} / s_{\alpha}\right)+\partial_{\alpha}\left((T-\mathbf{W} \cdot \hat{\mathbf{s}}) \gamma / s_{\alpha}\right) .
\end{aligned}
$$

Given $s_{\alpha}$ and $\theta$, the position $(x(\alpha, t), y(\alpha, t))$ is reconstructed up to a translation by direct integration of

$$
\left(x_{\alpha}, y_{\alpha}\right)=s_{\alpha}(\cos (\theta(\alpha, t)), \sin (\theta(\alpha, t)))
$$

which defines the tangent angle. The integration constant is supplied by evolving the position at one point $\mathbf{X}_{0}(t)$.

\section{(B). Small Scale Analysis}

Reformulate the equations by explicitly separating the dominant terms at small spatial scales. The behavior of the equations at small scales is important because stability constraints (i.e. stiffness) arise from the influence of high-order terms at small spatial scales. In HLS94, it is shown that at small scales the Birkhoff-Rott operator simplifies enormously. A useful notation, $f \sim g$, is introduced to mean that the difference between $f$ and $g$ is smoother than $f$ and $g$. In HLS94, it is demonstrated that

$$
U(\alpha, t) \sim \frac{1}{2 s_{\alpha}} \mathcal{H}[\gamma](\alpha, t)
$$


That is, at small spatial scales, the normal (physical) velocity is essentially the Hilbert transform with a variable coefficient. Now, Eq. (22) allows a rewriting of the equations of motion in a way that separates the dominant terms at small scales. We remark that these terms determine the stability constraints. Rewriting the equations, we obtain

$$
\begin{aligned}
\theta_{t} & =\frac{1}{2} \frac{1}{s_{\alpha}}\left(\frac{1}{s_{\alpha}} \mathcal{H}[\gamma]\right)_{\alpha}+P, \\
\gamma_{t} & =W e^{-1}\left(\frac{\theta_{\alpha}}{s_{\alpha}}\right)_{\alpha}+Q .
\end{aligned}
$$

Here, $P$ and $Q$ represent "lower-order" terms at small spatial scales. This is the small-scale decomposition (SSD). Assuming that $s_{\alpha}$ is given, the dominant small-scale terms are linear in $\theta$ and $\gamma$, but also nonlocal and variable coefficient. At this point, it is possible to apply standard implicit time integration techniques where the leading order "linear" terms are discretized implicitly. However, we have not yet taken any advantage in choosing the tangential velocity $T$. There are choices of $T$ that are especially convenient in constructing efficient time integration methods and in maintaining the accuracy of the simulations.

\section{(C). Special Choices for $T$}

Choose the tangential velocity $T$ to preserve dynamically a specific parametrization, up to a time-dependent scaling. In particular, require that

$$
s_{\alpha}(\alpha, t)=R(\alpha) L(t) \text { with } \int_{0}^{1} R(\alpha) d \alpha=1,
$$

where $R(\alpha)$ is a given smooth and positive function. The length $L(t)$ evolves by

$$
L_{t}=-\int_{0}^{1} \theta_{\alpha^{\prime}} U d \alpha^{\prime}
$$

If the constraint (25) is satisfied at $t=0$, then it is also satisfied dynamically in time by choosing $T$ as

$$
T(\alpha, t)=T(0, t)+\int_{0}^{\alpha} \theta_{\alpha^{\prime}} U d \alpha^{\prime}-\int_{0}^{\alpha} R\left(\alpha^{\prime}\right) d \alpha^{\prime} \cdot \int_{0}^{1} \theta_{\alpha^{\prime}} U d \alpha^{\prime}
$$

where the integration constant $T(0, t)$ is typically set to zero. This follows directly from Eqs. (18) and (26).

In HLS94/HLS97 different choices are used for $R$, and so for $T$. That which is computationally most convenient is $R \equiv 1$, yielding what is referred to as the uniform parametrization frame since a uniform discretization in $\alpha$ is then uniform in $s$, i.e. $s(\alpha, t)=\alpha L(t)$. In the uniform case, the leading order terms of small-scale decomposition, Eqs. (23) \& (24), are constant coefficient in space, and implicit treatments in time of these terms are directly inverted by the Fourier transform.

Since the uniform parametrization frame keeps computational points equally spaced in arclength everywhere along the curve, this frame can be deficient in capturing structures such as the blow-up in curvature that apparently occurs in the topological singularity. From Eq. (25), if $R<1$ in such a region, then there is a greater relative concentration of grid points there. Accordingly, in HLS97 [82], a nontrivial mapping $R$ is used to cluster computational points in regions of the curve where local refinement is needed. This yields the variable parametrization frame. The regions where local refinement is necessary are identified beforehand by examination of simulations using the uniform parametrization. The specific choice of $R$ is given in Appendix A in HLS97; an additional class of reference frames is also given in Appendix 2 of HLS94. 
For a nontrivial $R$, the leading order terms in the PDEs for $\theta$ and $\gamma$ are still linear, but are variable coefficient in space. Thus, in an implicit method, iterative methods are required to invert these terms to obtain the solution at the next time step. Because of the additional expense associated with solving the linear system, the variable parametrization frame is used in HLS97 only when it is crucial to obtain extra accuracy such as is the case at late times in the regions where (topological) singularities occur. As we will see in the next section, though, the expense of inverting the linear system, typically $O(N \ln N)$ per time step, is much less than the cost associated with an implicit treatment of the full system which is $O\left(N^{2}\right)$ per time step, due to the cost associated with evaluating the Birkhoff-Rott integral.

The use of the uniform or variable parametrization frames alone, without the $\theta-s_{\alpha}$ reformulation and an implicit treatment of the equations of motion, does in fact prevent $s_{\alpha}$ from becoming small as $s_{\alpha}$ now scales with the overall length of $\Gamma$. This removes the strong time-dependency in timestep restriction (17). However, the $3 / 2$ order constraint relating the time-step to the spatial grid size still remains. By using the $\theta-s_{\alpha}$ reformulation and the implicit treatment of the leading order terms, this higher-order constraint is removed as well, typically leaving only a first order Courant-Friedrichs-Lewy (CFL) type constraint from advection terms, appearing in both the $\theta$ and $\gamma$ equations, that are hidden in $P$ and $Q$.

Comment: The use of intrinisic coordinates to compute or simplify the dynamics of interfaces driven by their geometry has a long history. Examples include Whitham in his early work on shock propagation [212]; Brower, Kessler, Koplik, \& Levine in work on geometrical models of interface evolution [29]; Strain in work on unstable solidification [181]; Schwendeman in work on thermally driven motion of grain domain boundaries in crystallized solids [171]; Goldstein and his collaborators in work on the elastic, overdamped dynamics of polymers [65, 71]; Hou, Klapper, \& Si in work on the inertial dynamics of filaments [80]; and Shelley \& Ueda [175, 176] in work on dynamics arising in phase transitions of smectic-A materials.

\section{(D). Extension to the General Two Fluid Case}

When the interface $\Gamma$ separates two fluids with different densities, the Atwood ratio $A_{\rho}$ is nonzero. This means that a Fredholm integral equation of the second kind must be solved to obtain $\gamma_{t}$ due to the $\mathbf{W}_{t}$ term in Eq. (10). It turns out, however, that the small scale decomposition (23)-(24) remains valid. This may be seen explicitly by rewriting Eq. (10) as

$$
\gamma_{t}(\alpha, t)+K\left[\gamma_{t}\right](\alpha, t)=f(\alpha, t),
$$

where $K$ is the integral operator

$$
K\left[\gamma_{t}\right](\alpha, t)=-2 A_{\rho} \int \gamma_{t}\left(\alpha^{\prime}, t\right)\left[\frac{x_{\alpha}\left(y(\alpha, t)-y\left(\alpha^{\prime}, t\right)\right)-y_{\alpha}\left(x(\alpha, t)-x\left(\alpha^{\prime}, t\right)\right)}{\left|\mathbf{x}(\alpha, t)-\mathbf{x}\left(\alpha^{\prime}, t\right)\right|^{2}}\right] d \alpha^{\prime} .
$$

Observe that the kernel has a removable singularity at $\alpha=\alpha^{\prime}$. Thus, $K$ is smoothing at small spatial scales [81]. Further, $f(\alpha, t)$ in Eq. (28) contains all the terms in Eq. (10) which do not contain $\gamma_{t}$. Note that of these terms, $\kappa_{\alpha}$ is still dominant at small spatial scales. Since the integral operator $I+K$ is invertible for $\left|A_{\rho}\right| \leq 1$ [96], we may write the solution as

$$
\gamma_{t}=f-K\left[(I+K)^{-1} f\right] \text {. }
$$

Finally, since $K$ is smoothing at small scales, $\gamma_{t} \sim f \sim \kappa_{\alpha}$. This justifies the assertion above. For additional details, we refer the reader to Appendix 1 of HLS94. 


\subsection{Temporal and Spatial Discretizations}

Let us begin with the temporal discretizations described in HLS94/HLS97. The ODE (26) for $L(t)$ is not stiff. Therefore it may be solved using an explicit method. For example, using the 2nd order Adams-Bashforth method,

$$
L^{n+1}=L^{n}+\frac{\Delta t}{2} \int_{0}^{1}\left(3 \theta_{\alpha^{\prime}}^{n} U^{n}-\theta_{\alpha^{\prime}}^{n-1} U^{n-1}\right) d \alpha^{\prime}
$$

Consequently $L$ is always available at the $(n+1)$ st time-step. In HLS97, a fourth order method is also used to solve this ODE.

Next, consider the 2nd order Crank-Nicholson time discretization of Eqs. (23)-(24) in the uniform parametrization frame. The equations are discretized in Fourier space. Let $\hat{\theta}^{n}(k)$ denote the Fourier transform of $\theta$ at wavenumber $k$ and at time $t_{n}=n \Delta t$. Let $\hat{\gamma}^{n}(k)$ denote the analogous quantity. Then,

$$
\begin{aligned}
& \frac{\hat{\theta}^{n+1}(k)-\hat{\theta}^{n-1}(k)}{2 \Delta t}=\frac{|k|}{4}\left[\left(\frac{2 \pi}{L^{n+1}}\right)^{2} \hat{\gamma}^{n+1}+\left(\frac{2 \pi}{L^{n-1}}\right)^{2} \hat{\gamma}^{n-1}\right]+\hat{P}^{n}(k) \\
& \frac{\hat{\gamma}^{n+1}(k)-\hat{\gamma}^{n-1}(k)}{2 \Delta t}=-\frac{k^{2}}{2 W e}\left[\frac{2 \pi}{L^{n+1}} \hat{\theta}^{n+1}+\frac{2 \pi}{L^{n-1}} \hat{\theta}^{n-1}\right]+\hat{Q}^{n}(k),
\end{aligned}
$$

where we have used that $\hat{\mathcal{H}}=-i \operatorname{sgn}(k)$. The updates $\hat{\theta}^{n+1}$ and $\hat{\gamma}^{n+1}$ can then be found explicitly by inverting a $2 \times 2$ matrix. For details, see HLS94.

In HLS97, a $4^{\text {th }}$-order accurate, implicit, multi-step method due to Ascher, Ruuth, \& Wetton [8] is also used to discretize in time both the uniform and variable parametrization formulations. Using this method, Eqs. (23)-(24) may be reduced to the following single equation for $\theta^{n+1}$,

$$
s_{\alpha}^{n+1} \theta^{n+1}(\alpha)-\frac{1}{2 W e}\left(\frac{12}{25}\right)^{2} \Delta t^{2}\left(\frac{1}{s_{\alpha}^{n+1}} \mathcal{H}\left[\frac{\theta^{n+1}}{s_{\alpha}^{n+1}}\right]_{\alpha}\right)_{\alpha}=N(\alpha),
$$

where $N(\alpha)$ is a known quantity that depends on the solutions at the previous time steps. In the uniform parametrization case, $\theta^{n+1}$ is obtained explicitly by solving Eq. (34) in Fourier space since there the equation is diagonal. In the variable parametrization case, the discrete system is symmetric positive definite and is solved in physical space using the preconditioned conjugate gradient method. The application of $\mathcal{H}$ is performed in Fourier space, however, so that each step of the iteration requires $O(N \ln N)$ operations. The preconditioning operator $M$ is given by

$$
M\left(\theta^{n+1}\right)=s_{\max } \theta^{n+1}-\frac{1}{2 s_{\min } W e}\left(\frac{12}{25}\right)^{2} \Delta t^{2} \mathcal{H}\left[\theta_{\alpha}^{n+1}\right]_{\alpha \alpha},
$$

where $s_{\min }=\min _{\alpha} s_{\alpha}^{n+1}$ and $s_{\max }=\max _{\alpha} s_{\alpha}^{n+1}$. Thus, $M$ is constant coefficient and is diagonalized by the Fourier transform. For details, we refer the reader to Appendix B of HLS97.

In HLS94 and HLS97, spectrally accurate spatial discretizations are used in both the uniform and variable parametrization frames. Any differentiation, partial integration, or Hilbert transform is found at the mesh points by using the discrete Fourier transform. A spectrally-accurate alternatepoint discretization $[178,172]$ is used to compute the velocity of the interface from Eq. (8), i.e.

$$
\mathbf{u}_{i}=\frac{h}{\pi} \sum_{(i-j) \text { odd }} \gamma_{j} \frac{\left(\mathbf{X}_{i}-\mathbf{X}_{j}\right)^{\perp}}{\left|\mathbf{X}_{i}-\mathbf{X}_{j}\right|^{2}}
$$

where $\mathbf{u}_{i}=\mathbf{u}\left(\alpha_{i}\right)$ and $\alpha_{i}=i h$. The other variables in Eq. (36) are defined analogously. Finally, as noted in HLS94, time-stepping methods for vortex sheets suffer from aliasing instabilities since 
they are not naturally damping at the highest modes. The instability is controlled by using Fourier filtering to damp the highest modes and Krasny filtering [104] to remove round-off error effects; this determines the overall accuracy of the method, and gives a formal accuracy of $O\left(h^{16}\right)$. An infinite-order filter could also have been used.

Empirically, it is found that the time stepping routines discussed above remove the high order time step constraints due to surface tension and suffer only from a first order CFL time step restriction. In fact, the stability and convergence of these boundary integral methods has only recently been proved in the semi-discrete case in which time is continuous. For example, Beale, Hou \& Lowengrub (BHL96) [19] proved convergence of a class of boundary integral methods, using a slightly different formulation (based on the velocity potential and Bernoulli's equation) than that described above, in the context of water waves. Later, Ceniceros \& Hou (CH98) [33] proved convergence of a class of methods analogous to those described above in the case of interfacial flows between two liquids. An important feature of the BHL96 and CH98 stability analyses is that a certain compatibility between the choice of quadrature rule for the velocity integral (8) and the choice of spatial derivative must be satisfied on the discrete level in order to achieve numerical stability. This compatibility relation is subtle and ensures that a delicate balance of terms that holds on the continuous level is preserved on the discrete level. This balance is crucial for maintaining numerical stability and is one of the reasons why many previous investigations suffered from numerical instability. Using linear stability analysis near equilibrium interfaces, BN98 [11] independently also noted and removed instability due to the incompatibility of the operators.

While the BN98 result, and the spectrally accurate method described above, satisfy the compatibility relation, most straightforward implementations of the boundary integral equations do not. The compatibility condition is described most easily in the case of water waves without surface tension. In this case, the compatibility relation boils down to satisfying

$$
\Lambda_{h}(f)_{j}=\mathcal{H}_{h} D_{h}(f)_{j},
$$

for all discrete functions $f_{j}$ where $\Lambda_{h}$ is the discretization of

$$
\frac{1}{\pi} \int \frac{f(\alpha)-f\left(\alpha^{\prime}\right)}{\left(\alpha-\alpha^{\prime}\right)^{2}} d \alpha^{\prime}
$$

which is related to the first variation of the velocity integral (8). $\mathcal{H}_{h}$ is the discretization of the Hilbert transform using the same quadrature method as used for $\Lambda_{h}$. $D_{h}$ is the discrete derivative operator. Using alternate point quadrature to evaluate the velocity, the only compatible spatial discretization is $S_{h}$, the spectral derivative with the $N / 2$ mode set to zero. However, one may introduce appropriate Fourier filtering in the approximation of the velocity integral so that a version of Eq. (37) holds for all choices of $D_{h}$. Doing this appropriately, one obtains

$$
\Lambda_{h}\left(f^{\rho}\right)_{j}=\mathcal{H}_{h} D_{h}(f)_{j}
$$

where $\hat{f}^{\rho}(k)=\rho(k h) \hat{f}(k)$ and $\rho$ is defined by $\hat{D}_{h}=i k \rho(k h)$. In the case of surface tension and two liquids, additional filtering must be used to maintain compatibility for arbitrary derivatives; alternate point quadrature and the spectral derivative are always compatible. We refer the reader to BHL96 and CH98 for further details.

\subsection{The Kelvin-Helmholtz Instability, Surface Tension, and Singularities}

An interface separating two immiscible fluids is susceptible to the Kelvin-Helmholtz instability when shear develops across that interface. This is a fundamental instability of fluid mixing, such as 
that required to produce emulsions. Here, we study this problem in its simplest form and consider an interface separating two density-matched, inviscid fluids.

Fixing the initial data, the Weber number $W e$ alone controls the dynamics. For very small $W e$, the flow is dominated by surface tension effects, and linear theory gives no instability, predicting only oscillation. And indeed, the simulations in HLS97 show only an oscillatory dynamics welldescribed by linear theory, even over very long times. The dynamics becomes successively more interesting as the Weber number is increased, and the flow becomes more dominated by inertial effects, and hence, the Kelvin-Helmholtz instability. Fig. 3 shows the evolution from single-mode initial data, for two intermediate values of $W e$ at which only the $k=1$ mode is linearly unstable. The nonlinear outcome of the linear instability is fascinating: The interface elongates into long spikes and the two fluids interpenetrate. Our simulations suggest that this process can continue indefinitely, with the interface length eventually growing at a linear rate, with the finger width thinning exponentially. As $W e$ is increased and the number of linearly unstable modes increases, inertial effects become dominating. Fig. 4 shows the simulation of evolution for single-mode data (the same as above) for $W e=200$, for which there are 16 linearly unstable modes. The largescale spiral structure resembles what one expects from the development of the Kelvin-Helmholtz instability, based on the zero-surface tension simulations of Krasny [103] using vortex blobs.

There are two crucial points to make. First, with zero surface tension this initial data (see [104]) produces a Moore singularity at the center of the sheet approximately at time $t=0.375$. At large but finite $W e$ the shadow of this singularity is seen as short-wavelength dispersive waves erupting from the center of the developing spiral, and propagating outwards. Thus, surface tension disperses the Moore singularity in a manner reminscent of the dispersion of near-shocks in the KdV equation. The second point is that while a Moore-type singularity is avoided, the roll-up does not proceed smoothly onwards. As the latter stages of our simulation show, the roll-up appears to be terminated by the collision of interfaces within the interior of the spiral, that is, the smooth dynamics is punctuated by a collision, in finite time, of the sheet with itself in the inner turns of the spiral.

As is well-known, a collision of material interfaces in a flow implies the divergence of velocity gradients (see, e.g., HLS97). We refer to this collision as a topological singularity, since such collisions must precede a reconfiguration of fluid interfaces in a multi-phase flow (as in the pinch-off of a droplet). In HLS97, locally refined grids and high-order time-stepping are used, together with an SSD formulation, to very carefully isolate this oncoming topological singularity, and to study its analytical structure. This study reveals the following: As the spiral forms and disparate sections of the interface come in proximity to one another, a jet begins to form and intensify, fluxing fluid into the inner core of the spiral. This jet is associated with (i) the thin neck shown in close-up in Fig. 4f, and (ii) with the formation of oppositely signed sheet strengths (or interfacial vorticity) on the opposing sides of this neck. This creation of oppositely signed sheet strength is a direct consequence of surface tension as in its absence the sheet strength is conserved in the Lagrangian frame, and our initial data has sheet strength of a single sign. That the thickness of this neck falls to zero in a finite time is demonstrated in Fig. 5 (upper), which shows the neck width as a function of time.

The oncoming singularity bears some signs of self-similarity. Expectations of self-similarity would suggest that

$$
\text { Neck Width } \sim\left(t_{p}-t\right)^{\psi}
$$

where $t_{p}$ is the singularity time (its estimate is shown as the vertical dashed line in Fig. 5 (upper)), and $\psi=2 / 3$ the similarity exponent [95]. We find that the collapse follows this Ansatz quite closely. Our simulations suggest that both $\gamma$ and the interfacial curvature $\kappa$ are diverging as the 
collapse time is approached. However, we did not find that the rate of divergence was well fit by self-similarity $(\psi=-1 / 3$ for $\gamma$ and $-2 / 3$ for $\kappa)$. We did find strong evidence that the opposing interfaces were forming corners as the critical time was approached, which is also predicted by expectations of self-similarity. The lower part of Fig. 5 shows the successive blow-ups of the neck region as the singularity is approached, making the onset of a corner rather convincing.

Fig. 6 shows the evolution of the vortex sheet from more complicated, multi-mode initial data. This reveals that dynamics from more general initial data is composed of a combination of finger structures, as seen in Fig. 3, and spirals and pinches, as seen in Fig. 4. In HLS97, many more details are found concerning the simulations. These include numerical resolution studies, and further detail on the nature of the singularity, such as its behavior in the zero surface tension limit. On the latter, our results suggest that before the Moore singularity time of zero surface tension flow, surface tension acts as a regular perturbation.

We have also attempted to abstract from our simulations the basic phenomena underlying the pinching singularity, which is the collapse of a jet between interfaces under surface tension. Fig. 7 shows the direct collapse of a jet, with no intervening roll-up, between two interfaces under surface tension. The initial data was chosen so that only the $k=1$ mode was unstable, in crude analogy to the length-scale selection seen in the full simulations. The collapse of such jets is studied further, analytically and numerically, within long-wave approximations, by Pugh \& Shelley [148].

\subsection{The Rayleigh-Taylor Instability and Surface Tension}

Another classical hydrodynamic instability is the Rayleigh-Taylor instability (see e.g. [55] for a general reference), which occurs when a layer of heavy fluid sits on top of a lighter fluid. Such a situation may arise, for example, when a stably stratified multi-component fluid system is subjected to a destabilizing temperature gradient (or turned upside down).

Here, we consider again the simplest model: a single interface that separates two inviscid, immiscible fluids of different densities. In HLS94, the nonlinear evolution of such a dividing interface for unstably stratified fluids is simulated using the Boussinesq approximation. In that approximation, the density variation is taken only in the gravitational term. Its evolution showed the development of the classical Rayleigh-Taylor plume, with a developing local Kelvin-Helmholtz spiral. As for the Kelvin-Helmholtz instability discussed in the previous subsection, it appeared that its evolution was terminated by a collision within the turns of the spiral (Fig. 18 of HLS94). This problem has since been studied more comprehensively by Ceniceros \& Hou [33] (CH98).

CH98 investigated several examples of two-fluid interfacial flows in the presence of surface tension. Of particular interest are their high resolution numerical simulations of evolving, unstably stratified two-fluid interfacial flows, as shown in Fig. 8. This simulation, $W e=200, A_{\rho}=-0.1$, and $\mathrm{Fr}^{-1}=2.0$, was done using an SSD approach with the uniform parameterization frame. For $A_{\rho}>-1$ and without surface tension, the interface is known to develop a curvature singularity due to the Rayleigh-Taylor instability at an early time (a version of the Moore singularity, see [13]). Surface tension regularizes the Moore singularity and allows a smooth solution to exist beyond this early singularity time. By time $t=0.9$, two small fingers appear in the interface, and the interface begins to roll up. One can also see some capillary waves that are generated around $t=1.2$ and move outwards from the centers of roll-up. The finger tips broaden as they continue to roll up, and the interface bends towards the finger tips. This again is very much like the topological singularity formation seen in the Kelvin-Helmholtz case. As in that case, the minimum distance between the finger tip and the opposite side of the interface approaches to zero. Although CH98 did not use a variable parameterization frame for better resolution of the singularity, they found good agreement with the $\left(t_{c}-t\right)^{2 / 3}$ asymptotic form of the Neck Width obtained in HLS97. 
The study of CH98 seems to indicate that the topological singularity observed in HLS94/HLS97 for a vortex sheet with surface tension is quite generic. In addition, a recent study by Tryggvason \& Univerdi (TU98) [196] provides further evidence of the genericity. For example, TU98 report that interfaces between two immiscible fluids, with small but finite viscosities, exhibit similar structures under shear and buoyancy as do their inviscid counterparts described here and in HLS94, HLS97 and CH98.

\subsection{Dynamic generation of capillary waves}

A familiar example of interfacial flows which are not driven by a hydrodynamic instability is the motion of water waves. Water waves are the source of many interesting nonlinear phenomena such as wave-breaking, and the dynamical generation of capillary waves on the forward wave front $[57,59,142]$. These capillary waves typically appear near the crest of the main wave where the local curvature is very large and surface tension becomes important. The understanding of these short waves is important in the remote sensing of sea surface because the fine structure associated with short wavelengths scatters electromagnetic radiation. Capillary waves are also believed to be a mechanism for extracting energy from wind-generated waves and may be significant in wave breaking $[57,59]$.

There have been many thorough studies of capillary waves generated by steady steep gravity waves (see e.g. $[118,119,44]$ and the references cited there). For unsteady capillary waves, Tulin [199] has performed a careful numerical study on the effects of surface tension on breaking waves. Tulin's simulations show the appearance of a capillary jump, as defined by Longuet-Higgins [119], near the wave crest. More detailed structure of capillary waves has been revealed recently by Ceniceros and Hou (CH99a) [34]. Using the methods of HLS94/HLS97, they are able to compute with high accuracy not only up to the appearance of the capillary jump as observed by Tulin, but also to follow the subsequent development of small-scale structures.

An example of this type of behavior is seen in Figs. 9 and 10. In this simulation, $W e=10^{3}$, $\mathrm{Fr}^{-1}=-20$, and an initial shear is imposed to give the wave the impulse to overturn. One can see that the interface becomes vertical at $t=0.30$ and a capillary wave appears soon after the wave begins to overturn. Fig. 10 (a) gives a close-up of a neighborhood of the crest at time $t=0.45$. The curvature in this region is plotted in Fig. 10(b), in which a capillary wave train can be clearly seen. By a close inspection of the onset of this capillary wave at $t=0.26$, the curvature is seen to develop a spike right behind the wave tip. However, such a spike is not present in the corresponding zerosurface-tension solution for which curvature varies smoothly in the entire region [34]. Soon after this time, this spike develops into the capillary wave train. Note also that the capillary intrusion gets narrower in time (see Fig. 10(a)), suggesting that a small gas bubble will soon be pinched off and trapped within the fluid.

A useful quantity to consider is the so-called capillary wavelength $\lambda_{W e}$, defined as the distance between the two largest values of $|\kappa|$. Although it is difficult to obtain an accurate scaling for $\lambda_{W e}$, the CH99a study suggests that for a fixed time, both the capillary wavelength and its amplitude decrease nonlinearly as $W e^{-1}$ decreases to zero. The scaling for $\lambda_{W e}$ is roughly $O\left(W e^{-1 / 2}\right)$. This seems to be in agreement with the experimental results of Duncan et al. [57]. Moreover, for this data, CH99a find that the interface profiles with decreasing surface tensions converge to the zero-surface-tension profile at the fixed time $t=0.45$. This is not surprising since the limiting zero-surface-tension water wave problem is well-posed, even after the wave overturns [213]. Here surface tension acts as a regular perturbation. However, this conclusion does not apply to the ill-posed Hele-Shaw problem as we will see in Sec. 3. 


\section{Hele-Shaw Flows}

Hele-Shaw flow is the quasi- two-dimensional flow of a viscous fluid between two closely spaced plates - the so-called Hele-Shaw cell [77]. Driven multi-fluid flows in the Hele-Shaw cell have been intensely studied, both experimentally and theoretically, because the nonlinear development of the Saffman-Taylor (S-T) instability [165] can lead to prototype "densely branched" patterns, and is one of a class of pattern-forming systems that includes crystal growth, electro-deposition, bacterial growth, and directional solidification. A recent review of the many experimental perturbations of Hele-Shaw flow is given by McCloud \& Maher [124]. Hele-Shaw flows also give a relatively simple and well-characterized setting in which to study the effects (often subtle) of surface tension on the development of singularities, pattern selection, and as a physical regularization of an ill-posed system. (Like the Kelvin-Helmholtz system, Hele-Shaw flows can have linear growth rates that scale linearly with wave-number.) A much better theoretical understanding of these problems has followed from analysis, guided or validated by highly accurate and efficient simulations. A recent review of the role of surface tension in Hele-Shaw flows is given by Tanveer [185]. A comprehensive bibliography on Hele-Shaw and Stokes flows has been compiled by Gillow \& Howison [63].

The usual starting point for theoretical investigations of Hele-Shaw flows is Darcy's law. Consider two incompressible, viscous, and immiscible fluids in a Hele-Shaw cell, separated by a planar interface $\Gamma$. As before, let $j=1$ or 2 label the two fluids. In a Hele-Shaw cell, the (gap-averaged) velocity of each fluid $(j)$ is given by Darcy's law, together with the incompressibility constraint:

$$
\mathbf{u}_{j}(x, y)=-\frac{b^{2}}{12 \mu_{j}}\left(\nabla p_{j}(x, y)-\rho_{j} \mathbf{F}(x, y)\right), \quad \nabla \cdot \mathbf{u}_{j}=0
$$

where $b$ is the cell gap width, $\mu_{j}$ the fluid viscosity, $\rho_{j}$ its density, and $F=-\nabla \Phi$ is a body force (usually divergence free, e.g. gravitational). Boundary conditions typically used are the kinematic and dynamic boundary conditions as given in Eqs. (2) \& (3). These are augmented by far-field boundary conditions on the velocity or pressure. Taking the divergence of Eq. (39) shows that $p$ is harmonic, which is the basis from which most numerical treatments proceed.

\subsection{Historical Perspective}

Many different numerical approaches have been applied to simulating Hele-Shaw flows. These include volume-of-fluid methods (e.g. [210, 100]), boundary element methods (e.g. [75]), level set and immersed boundary methods [86], and statistical methods based on diffusion limited aggregation (e.g. [116, 7]). Methods based on conformal mapping have long been used to study dynamics of Hele-Shaw flows (see [23, 45, 185] for reviews and references). However, conformal mapping methods apply most naturally to singly-connected domains, and can have difficulties with efficiently including the effect of surface tension. As a numerical method, the most sophisticated version of conformal mapping seems that due to Baker, Siegel, \& Tanveer [12], who solve a well-posed evolution problem for zero surface tension by analytically continuing initial data and equations of motion into the complex plane, and explicitly tracking the solution's poles and other singularities.

Due to their natural applicability, flexibility, and potential for high accuracy, boundary integral methods have developed into a powerful method for simulating Hele-Shaw flows. Their first application to study dynamics seems to be due to Tryggvason \& Aref [194, 195], who in a highly ambitious work studied two fluid mixing via a Rayleigh-Taylor instability, and the interaction of S-T fingers. Posing the interfacial velocity in terms of a vortex sheet, they gave an integral equation of the $2^{\text {nd }}$ kind for its strength $\gamma$, essentially of the form in Eq. (28) (which is for $\gamma_{t}$ ). The integral equation was solved via iteration (similar to [14] for inviscid waves), coupled to a vortex-in-cell 
(VIC) approach for the rapid evaluation of the Birkhoff-Rott integral. In studying mixing, they were able to achieve considerable ramification of the interface, though this was likely aided by the smoothing of VIC methods. This work was soon followed by Davidson [47, 48] and DeGregoria \& Schwartz [50, 51, 52]. In [47], Davidson posed a boundary integral representation, and used it subsequently [48] to study the development of S-T fingers to modest amplitude. DeGregoria \& Schwartz studied various aspects of tip-splitting and the stability of S-T fingers. Their boundary integral approach was coupled to a grid redistribution strategy that kept computational points in regions of high curvature, and used a a stiff ODE solver to reduce the stiffness from surface tension. Meiburg \& Homsy [127] employed a vortex sheet description, with the interface discretized as circular arcs, to study aspects of splitting and finger instability. Following Dai, Kadanoff, \& Zhou [45], who used conformal mapping methods, Dai \& Shelley [46] applied boundary integral methods (discretized to infinite order) to study regularization of Hele-Shaw flows by surface tension, and also the interaction of surface tension and noise. They partially ameliorated the stiffness of surface tension by choosing a dynamical frame that kept computational points almost uniformly spaced. They also applied Krasny filtering, [104] to control growth of round-off errors in their simulations. Using a vortex sheet representation, Whitaker [211] compared the effect of different spatial discretizations on simulating propagating fingers. Power [144] used a boundary integral representation to simulate the initial development of the S-T instability for two fluids in a radial geometry.

\subsection{The Small-Scale Decomposition for Hele-Shaw Flows}

In HLS94 [81], we developed the SSD for Hele-Shaw flows (described below). This efficiently subverted the stiffness due to surface tension, and as with inertial vortex sheets, has allowed the accurate and long-time simulation of many prototypical Hele-Shaw flows. Further, given the close analogy of Hele-Shaw flow to solidification models in materials science, much of the numerical technique is immediately applicable there (see next section).

That the velocity field has the form given in Eq. (5) follows from Darcy's law (39) (which implies that the velocity $\mathbf{u}_{j}$ is irrotational), the incompressibility constraint, and from the kinematic and dynamic boundary conditions. An equation for $\gamma$ follows from these, together with the LaplaceYoung condition; see [194] or [46] for details. In nondimensional variables, $\gamma$ satisfies

$$
\gamma=-2 A_{\mu} s_{\alpha} \mathbf{W} \cdot \hat{\mathbf{s}}+S \kappa_{\alpha}+R \mathbf{F} \cdot \hat{\mathbf{s}} .
$$

Here $A_{\mu}=\left(\mu_{1}-\mu_{2}\right) /\left(\mu_{1}+\mu_{2}\right)$ is the Atwood ratio of the viscosities, $S$ is a nondimensional surface tension, and $R$ is a signed measure of density stratification $\left(\rho_{1}<\rho_{2}\right.$ implies $\left.R<0\right)$. Due to the presence of $\gamma$ in the velocity $\mathbf{W}$, Eq. (40) is a Fredholm integral equation of the second kind for $\gamma$, and is, in general, uniquely solvable (see [14]).

For Hele-Shaw flows, the effect of surface tension is dissipative at small scales, and gives a higher order time-step constraint than for inertial flows. Again using a "frozen coefficient" analysis of the equations of motion, Beale, Hou \& Lowengrub [21] showed that least restrictive stability time-step bound on an explicit integration scheme was the time-dependent constraint

$$
\Delta t<C \cdot\left(\bar{s}_{\alpha} h\right)^{3} / S \text { where } \bar{s}_{\alpha}=\min _{\alpha} s_{\alpha} .
$$

This is a much stricter constraint than that for the inertial case (Eq. (39)). Again, the stability constraint is determined by the minimum grid spacing in arclength - perhaps strongly and adversely time-dependent - but the bound in terms of $\bar{s}_{\alpha}$ is also quadratically smaller. The lack of robust and efficient methods for subverting such constraints has severely limited simulations of Hele-Shaw and related flows. 
For the Hele-Shaw case, in HLS94 the small-scale analysis shows that the equations of motion can be given in the form:

$$
\theta_{t}=\frac{S}{2} \frac{1}{s_{\alpha}}\left(\frac{1}{s_{\alpha}} \mathcal{H}\left[\frac{\theta_{\alpha}}{s_{\alpha}}\right]_{\alpha}\right)_{\alpha}+N(\alpha, t)
$$

where, as in Eq. (23), the term dominant at small length-scales is separated out, and $N$ is the remaining, lower-order, terms.

The majority of simulations of Hele-Shaw flow have used $R \equiv 1$, which removes the variable coefficient nature of Eq. (42). Then, Eq. (42) simplifies to

$$
\theta_{t}=\frac{S}{2}\left(\frac{2 \pi}{L}\right)^{3} \mathcal{H}\left[\theta_{\alpha \alpha \alpha}\right]+N(\alpha)
$$

Eq. (43) is posed together with Eq. (26), the ODE for evolving $L$, and is a complete specification of the interfacial problem, with the highest order, linear behavior prominently displayed. This term is now diagonalizable by the Fourier transform, and so

$$
\hat{\theta}_{t}(k)=-\frac{S}{2}\left(\frac{2 \pi}{L}\right)^{3}|k|^{3} \hat{\theta}(k)+\hat{N}(k) .
$$

Implicit time integration methods can now be easily applied. As an example, consider linear propagator methods, which factor out the leading order linear term prior to discretization. They usually provide stable, even high-order, methods for integrating diffusive problems. The first use of such a method (of which the authors are aware) is by Rogallo [157] in simulations of the NavierStokes equations, though it has been rediscovered and used by several researchers in different contexts. For Hele-Shaw, Eq. (44) is rewritten as

$$
\frac{\partial}{\partial t} \psi(k, t)=\exp \left(\frac{S}{2}(2 \pi|k|)^{3} \int_{0}^{t} \frac{d t^{\prime}}{L^{3}\left(t^{\prime}\right)}\right) \hat{N}(k, t)
$$

where

$$
\psi(k, t)=\exp \left(\frac{S}{2}(2 \pi|k|)^{3} \int_{0}^{t} \frac{d t^{\prime}}{L^{3}\left(t^{\prime}\right)}\right) \hat{\theta}(k, t) .
$$

Eq. (45) follows from Eq. (44) by finding an integrating factor to incorporate the linear term into the time derivative. It is now Eq. (45) that is discretized using the $2^{\text {nd }}$ order Adams-Bashforth method. In terms of $\hat{\theta}$, the result is

$$
\hat{\theta}^{n+1}(k)=e_{k}\left(t_{n}, t_{n+1}\right) \hat{\theta}^{n}(k)+\frac{\Delta t}{2}\left(3 e_{k}\left(t_{n}, t_{n+1}\right) \hat{N}^{n}(k)-e_{k}\left(t_{n-1}, t_{n+1}\right) \hat{N}^{n-1}(k)\right)
$$

where $t_{n}=n \Delta t$, and

$$
e_{k}\left(t_{1}, t_{2}\right)=\exp \left(-\frac{S}{2}(2 \pi|k|)^{3} \int_{t_{1}}^{t_{2}} \frac{d t^{\prime}}{L^{3}\left(t^{\prime}\right)}\right) .
$$

The use of "linear propagator" is now clear; $\hat{\theta}$ at the $n^{\text {th }}$ time-step is propagated forward to the $(n+1)^{s t}$ time-step at the exact exponential rate associated with the linear term. If $N \equiv 0$, this yields the exact solution to the linear problem. Of course, the factor $e\left(t_{1}, t_{2}\right)$ still has a continuous time dependence through the presence of integrals. These integrals are evaluated by evolving auxiliary ODEs for the integrand, and for $L(t)$. Clearly, linear propagator methods can be formulated with high-order methods. 


\subsection{Pattern Formation}

Hele-Shaw flows can give rise to the formation of beautiful patterns - this is one aspect of the physicist's and mathematician's interest in them. In this section, we briefly discuss several scenarios and simulations of pattern formation.

From HLS94, Fig. 11 shows the simulation of a gas bubble expanding outwards into a Hele-Shaw fluid (see [45] and [46]) over long-times. From the competition of surface tension with the fluid pumping, this simulation shows the development of ramification through successive tip-splitting events - the S-T instability - and the competition between adjacent fingers. This simulation is also spectrally accurate (infinite order) in space, and uses a second-order in time linear propagator method for integrating the small-scale decomposition. There are no high-order time-step constraints. The fluid velocity is evaluated from the discretized Birkhoff-Rott integral in $O(N)$ operations using the Fast Multipole Method of Greengard \& Rokhlin [74]. The integral equation for $\gamma$ (arising from the viscosity contrast) is solved via the iterative linear system solver GMRES, using an SSD based preconditioner[163, 72]. The operation count is $O(N)$ at each time-step, where $N$ is the number of points describing the boundary. Here $N=4096$ and $\Delta t=0.001$. This time step is $10^{3}$ times larger than that used by Dai \& Shelley [46] in computations of a similar flow using an explicit method with a lesser number of points, and the interface here has developed far more structure.

A very different manifestation of the S-T instability and pattern formation is seen in Fig. 12. This simulation, due to Shelley, Tian, \& Wlodarski (see [174]) and using SSD based methods, shows the atypical patterns that can form at the liquid/gas interface that bounds a blob of viscous fluid, as the upper plate of a Hele-Shaw cell is lifted. This lifting puts the fluid blob under a lateral straining flow, sucking in the interface and causing it to buckle. This basic mechanism, though coupled to a much different material rheology, is likely responsible for producing the permanent patterns left behind after pulling up of some adhesive tapes. The resulting short-lived patterns can resemble a network of connections with triple junctions. A likewise odd pattern formation is seen in Fig. 13, from Lowengrub \& Shelley (1999, unpublished), which shows the nonlinear development of the S-T instability on a liquid bubble in a spinning Hele-Shaw cell (see [32] for related experiments). Here the central bubble throws out attached droplets of fluid, which then themselves become susceptible to the S-T instability, throwing out fingers which will perhaps themselves form new droplets. Such flows are relevant to the manufacturing process of spin coating, where it is of interest to control such instabilities.

In a set of beautiful simulations that sought to establish a concrete connection between HeleShaw flows and dendritic solidification, Almgren, Dai, \& Hakim [4] (ADH) used an SSD formulation to compute the long-time growth of "dendrites" in a Hele-Shaw flow with anisotropic surface tension. Fig. 14 shows a sample simulation from this work. As in the simulation from HLS94 described above, this shows an expanding gas bubble, but with the pressure boundary condition $p=d_{0}(1-\epsilon \cos m \Theta) \kappa$ where $\Theta$ is the azimuthal angle and $m$ an integer ( $m=4$ in the figure). (In their numerical implementation of the SSD, the "small-scale" terms use only the mean surface tension $d_{0}$, which at large anisotropies $\epsilon$ somewhat reduces the effectiveness of the method.) Now one sees the suppression of tip-splitting, and the formation of a densely branched pattern, replaced by "dendrites" traveling along directions of least surface tension, and shedding side-branches. Through their simulations and analysis, ADH established that the emerging finger displayed a simple temporal scaling, which has lead to new experimental and theoretical work in solidification and Hele-Shaw flows (see, e.g., [89, 146]).

Finally, we close this section with the remark that while the SSD analysis arises from the boundary integral representation, its application is not bound to it. Fig. 15 shows a simulation by 
Fast \& Shelley [60, 62] of the nonlinear development of the Saffman-Taylor instability for a weakly "shear-thinning" fluid. A mathematical description of a Darcy's law for shear-thinning fluids can be found in $[101,102,61,60]$, but suffice it to say that now the pressure is not harmonic, and has no boundary integral representation. To advance the interface the pressure must be found in the entire fluid domain at each time-step. Fast \& Shelley apply overset grid methods, based on the Overture framework [30], where the domain away from the interface is discretized by a fixed grid, and that near the interface by a narrow conforming grid. This avoids the difficulties of global grid generation, and minimizes grid anisotropy errors. An SSD formulation is used to control, again, the stiffness induced by surface tension. This simulation illustrates how shear-thinning leads to a relative suppression of the tip-splitting that underlies the densely branched morphology of Newtonian patterns (see also $[102,143]$ and the references therein).

\subsection{Singularity Formation}

In this subsection, we will review several recent applications of boundary integral methods to study the formation of singularities in Hele-Shaw flows. Singularity formation for Hele-Shaw flows without surface tension is practically generic. The modification or generation of singularities by the presence of surface tension is much less well understood. In what seems to be the first such result, Duchon and Robert [56] proved the short-time existence of a linearly unstable Hele-Shaw flow with surface tension (for a flow with suction from a mass sink). A long-time existence result has been obtained by Constantin and Pugh [41] for the linearly stable case of a near circular blob of fluid (with no suction). Recent simulational work has considered the formation of topological singularities (i.e. interfacial self-collision), and the collision of an interface with a mass sink.

\subsubsection{Topological singularities}

Topological transitions in fluid systems are poorly understood, and Hele-Shaw flows provide a simple, but nontrivial, situation in which to study them. The majority of studies concern analytical and numerical studies of simplified "lubrication", or long-wave, descriptions of Hele-Shaw flows, which yield nonlinear, but local, PDEs for the layer thickness (e.g. see [173, 39, 58, 67, 24, 68, 3, 25, 69, 70]. Two studies that have sought to study topological singularities in the full Hele-Shaw problem are Shelley, Goldstein, \& Pesci [173], and Almgren [2], who both applied boundary integral methods.

Shelley et al. [173] give an initial study of topological singularity formation in Hele-Shaw flows, where a thin fluid layer is being driven to rupture by a Rayleigh-Taylor instability. In this study, they develop a lubrication reduction from a long-wave expansion of the boundary integral description, and perform simulations that compare the approximate and full systems (see Goldstein, Pesci, \& Shelley [67, 68, 69, 70] for subsequent analytical and numerical studies of the associated lubrication PDE). For the full Hele-Shaw flow, Fig. 16 shows a simulation of the collapse of a thin fluid layer. Rising "spikes" of light fluid are accompanied by falling spikes of heavy fluid. The latter appears to collide at a finite time with the bottom wall (which can also be interpreted as collision with an interface rising from below as suggested in the lower figure). This again is a topological singularity, which necessarily is associated with a divergence, at the least, of flow velocity gradients. These simulations, and those of the associated lubrication PDE, suggested a divergence in $\kappa_{s}$, the interfacial derivative of curvature, with $\kappa$ remaining bounded. However, by using an explicit time-integration scheme these simulations employ only 129 spatial points because of the stiffness from surface tension. And so, while the spatial accuracy was of infinite order, the resolution was far short of that necessary capture reliably the details of the singularity. It was this simulation that 
originally motivated the development of the Small-Scale Decomposition.

As said above, Constantin and Pugh [41] have proven the long-time existence of smooth solutions for the Hele-Shaw flow of a blob of fluid initially close to being circular. They also show that a circular blob is asymptotically stable, with these solutions relaxing to it. Almgren [2] has given strong numerical evidence that their result does not hold far from equilibrium. He considered the evolution of singly connected blobs that initially had a dumbbell shape, with a thin flat neck of fluid connecting the two halves. His conjecture was that in the process of the domain seeking to minimize its interfacial length - this is a curve-shortening dynamics - the neck could collapse in width, forming a flow singularity. Unlike the problem considered above, this flow is "unforced" as there is no source of instability, such as a density stratification or mass source, and is driven purely by the surface tension at the boundary. To study this numerically, Almgren employs an SSD formulation, with a variable parameterization frame (Eq. (25)) that clusters computational points in the neck region. Rapid evaluation of the spatial interactions is done via the Fast Multipole Method [74], with GMRES [163] used to solve the integral equation for the vortex sheet strength. His simulations appear to confirm his conjecture, with the apparent singularity similar to that suggested above by the simulations of Shelley et al., and in agreement with the predictions of lubrication theory.

\subsubsection{Hele-Shaw flow with suction}

Consider a blob of fluid in a Hele-Shaw cell that contains a point mass sink that removes fluid at a constant rate. In this case, all of the fluid will be removed within a finite time, giving an upper bound on the time of existence of the flow. The question is: Does anything interesting happen beforehand? For example, might the bounding interface collide with the mass sink first, giving a singularity? For zero surface tension it has been shown that prior to the fluid being completely removed, the bounding interface can form cusps, or collide with itself, or reach the sink, and that only a circular blob with sink at its center does not develop singularities [79]. For nonzero surface tension, Howison, Lacey \& Ockendon have attempted a perturbation analysis of such a flow, using the knowledge of the zero-surface-tension solutions [87]. Assuming that surface tension is only important where curvature is large, they posit the existence of a self-similar steady-state solution (where on an inner scale surface tension is rescaled to be order one). Their analysis predicts that small surface tension could cause the interface in the neighborhood of the cusp to propagate rapidly as a narrow jet, analogous to a thin crack. However, the existence of such a self-similar steady-state solution is unknown, and the effects of very small surface tension past the cusp singularity time remained unclear. For nonzero surface tension, Tian [192] has shown that a singularity must occur if the mass sink and center of mass of the fluid domain do not coincide. The form of this singularity is not identified through his analysis.

Kelly and Hinch [97] studied numerically the effects of surface tension on a Hele-Shaw flow with suction. Using a boundary integral method ([98]; $2^{\text {nd }}$-order in space and time, with explicit timestepping) they showed that the cusp of the zero-surface tension solutions was avoided. However, their simulations are limited by the modest spatial resolution (200 grid points).

Nie and Tian [137] have performed a more resolved numerical study of the interface dynamics of a Hele-Shaw flow with suction. To reach high spatial resolutions (up to 4096 points) and accuracy, they use a spectrally accurate SSD formulation, with a Crank-Nicholson time discretization. Beginning with data that for zero surface tension would form a finite-time cusp (away from the sink), they find that surface tension induces a collision of the interface with the sink, with the interface

forming a corner at the instant of impact. They also provide a resolution study of their simulations in the neighborhood of the singularity, and suggest that the sink is approached by the interface as 
a square-root in time.

Ceniceros, Hou, and Si [36] (CHS) have provided a subsequent, yet more comprehensive study, considering both the asymptotic behavior as surface tension tends to zero, and the effect of having an external fluid - the so-called Muskat problem. Their numerical approach is also based on the SSD formulation coupled to $4^{\text {th }}$-order implicit and stable integration scheme [8]. Because of the ill-posedness of the zero surface tension limit, they control the spurious growth of round-off errors using Krasny filtering [104]. They also successively double the number of points, up to 16384, whenever the Nyquist frequency begins to rise above the filter level (usually $10^{-12}$ ).

CHS have found several new and intriguing results. They consider an initially circular blob of viscous fluid surrounded by inviscid fluid, i.e., $A_{\mu}=1$. Fig. 17 shows one such simulation from CHS, for surface tension $S=5 \times 10^{-5}$, with the interface initially a circle with the sink displaced upwards from its center. It shows the formation of the corner singularity as the interface collides with the sink, in agreement with Nie \& Tian. All of the graphs are at times beyond $t_{c}$, the time of a cusp singularity for zero surface tension with this particular data. One can clearly see that the "finger" bulges outwards, developing a well-defined neck before it forms a wedge. This neck appears at a height close to that of the zero-surface-tension cusp.

From the simulations of CHS, Fig. 18 illustrates the asymptotic trend of the singularity structure as surface tension is successively halved from $S=8 \times 10^{-4}$ to $S=5 \times 10^{-5}$. Here the different simulations are compared by setting the tip height above the sink at $y=0.01$. This figure, and their data analysis of the corner angle, strongly suggests that an asymptotic corner angle is selected in the limit of zero surface tension.

Other results for this class of singular flows can be found in CHS. For example, the simulations of CHS suggest that the small surface tension solution converges strongly to the zero-surface-tension solution before its cusp time $t_{c}$. But what happens after $t=t_{c}$, but before the interface reaches the sink? Their simulations suggest that the limiting finger has a rounded end (it hasn't yet reached the sink), but with a neck formed by two indented corners. The beginning of these limiting corners can be seen in Fig. 18. These results seem at odds with the predictions of the crack model of Howison et al. [87], and further details of this comparison are found in CHS [36]. CHS also study the twophase Hele-Shaw problem $\left(A_{\mu}<1\right)$, finding that larger viscosity in the exterior fluid prevents the formation of the neck, leads to the development of thinner fingers, and that the asymptotic wedge angle apparently decreases towards zero (becoming cusp-like) as the viscosities approach equality $\left(A_{\mu}=0\right)$.

It is natural to ask how the behavior of the interface would be in the corresponding 3-D Darcy flow. This has been studied recently by Ceniceros and Si [37], who find the situation very similar to that of 2-D Hele-Shaw flow (surprisingly, the azimuthal component of surface tension did not appear to play a dominant role, and no "pinching" singularities were observed).

\subsection{Finger selection and noise effects in Hele-Shaw cells}

As illustrated above, morphological instabilities are common to pattern formation problems. The non-equilibrium growth of crystals and directional solidification are some of the best known examples. Due to the underlying Mullins-Sekerka instability, the system is very sensitive to small perturbations and can originate convoluted interfacial patterns. The generic mechanisms in the formation of these complex patterns are present in the simpler problem of Hele-Shaw, and a fundamental question is what is the role of surface tension in the formation of these patterns. It can be surprisingly subtle.

In the absence of surface tension, many exact analytic solutions are known for Hele-Shaw flows. It is natural to use the knowledge of these exact solutions to perform a perturbation analysis around 
these solutions. Intuitively, one might think that so long as the zero surface tension solution exists and is smooth, the small-surface-tension solution would converge to it in the limit of zero surface tension. However, this intuition must confront the fact that the zero surface flows can be ill-posed (or rather, well-posed only for analytic initial data in the space of appropriate analytic norms), and that small perturbations will generically lead to singularity.

These considerations lead Dai \& Shelley [46] to numerically study whether surface tension acted as regular perturbation to a smooth, but unstable, zero surface tension flow (see also [45]). They investigated the influence of precision and its associated round-off errors - computing in up to 30 digits of precision - and separated those flow structures that seemed intrinsic to surface tension from those induced through the growth of round-off errors (see also [4] on the question of sidebranching). For their initial data, they concluded that surface tension was indeed acting as a regular perturbation.

However, the asymptotic theory of Tanveer [184, 185] and Siegel, Tanveer, \& Dai [179] suggests that small surface tension could be a singular perturbation for certain other initial data, even if the limiting zero-surface-tension solution were perfectly smooth. The reason is that surface tension, through the curvature, can introduce a new analytic structure (the so-called daughter singularity) at $t=0^{+}$which could subsequently impinge upon the physical domain by a time finite and independent of the surface tension. That is, for such initial data the limiting solution after a finite time would not be the zero surface tension solution. If true, this is a profound result both for the mathematical understanding of surface tension and its effects, and for the more general study of regularizations of ill-posed systems or systems yielding singularity.

In their simulational studies, Siegel et al. have given strong evidence supporting these analytical predictions. In part, these studies were done using a boundary integral method, with an SSD formulation for the dynamics [81], as well as conformal mapping methods as in Dai \& Shelley [46], to achieve high resolution for the interfacial flow. Unfortunately, because of the extreme sensitivity of the evolution to round-off errors, Siegel et al. find it very difficult to compute for very small surface tensions $\left(S<10^{-5}\right)$, even if quadruple precision (30 digits) is used.

For this class of initial data, Ceniceros \& Hou [35] (CH99b) have developed a sophisticated numerical approach to investigate the limiting behavior for small surface tension. Their method relies on three observations. The first is that noise can be significantly reduced by using a parameterization that yields a compact representation of the solution in Fourier space. Out of the many choices (Lagrangian, equal-arclength, etc.), it is the conformal mapping representation that gives the most compact parameterization of the interface. This compact parameterization was used likewise by Dai \& Shelley [46] and by Siegel et al. [179] in their previous studies. An advantage of using the conformal map formulation [40] is that the evaluation of the velocity integral can be done via Fast Fourier Transform in $O(N \log (N))$ operations. Further, it allows Krasny filtering [104] to be used very effectively to separate noise from the physical solution for the majority of high to intermediate modes.

The second observation is that the daughter singularity, born at $t=0^{+}$, is of amplitude $O(S \cdot t)$ for small times. Therefore, initially it requires many digits to capture the spectral property of this complex singularity in the physical domain. To alleviate this difficulty, they derive a scaled equation wherein the amplitude of the daughter singularity is $O(1)$ and can then be captured more effectively.

The third observation is that one needs to perform a resolution study in the precision and filter level, and to compute in very high precision for small surface tension (see also Dai \& Shelley [46], and Shelley [172] for related studies). At small surface tensions, very high precision arithmetic is used to solve numerically the scaled equation (up to 80 digits, using the multi-precision package of Bailey [9]). High precision has the additional benefit of reducing the amplitude of round-off error. 
Each of these ingredients is essential to simulating the dynamics accurately at the very small surface tensions considered in CH99b (4 orders of magnitude smaller than those used by Siegel et al. ). Consequently, CH99b have provided much stronger support for surface tension being a singular perturbation to Hele-Shaw flow. We summarize now the numerical study of CH99b. Following Siegel et al. [179], they choose as initial data an expanding bubble with three-fold symmetry (see Fig. 11 of [179]), and for which the zero-surface-tension solution forms three cusp singularities at $t_{c}=0.3301$. According to the asymptotic theory [184, 179], surface tension should produce an $O(1)$ perturbation from the zero-surface-tension solution around the much earlier time $t_{d}=0.0463$ - the daughter singularity impact time, found by solving an ODE [184] - no matter how small the surface tension, and even though the zero-surface-tension solution is still smooth at $t=t_{d}$.

Figs. 19 and 20 show some of the results from their study. For $S=10^{-8}$, Fig. 19 shows the evolution of the interfacial curvature, in the "tip" region (where the cusp would form for $S=0$ ), at times near $t_{d}$. Here the effect can be seen of the daughter singularity on the physical domain. At $t=0.043$, the tip curvature flattens in a very localized finite region, and from $t=0.046$ onwards, the curvature develops growing deviations from the zero-surface-tension curvature, with the affected physical region near the tip spreading in time. This behavior is consistent with the asymptotic theory which implies that the daughter singularity cluster will disperse once it gets sufficiently close to the unit disk. Fig. 20 shows the interfacial curvature at $t=0.048$, from the three simulations with surface tensions $S=10^{-7}, 10^{-8}$, and $10^{-9}$, as well as for the exact zero surface tension solution. The singular nature of the zero surface tension limit can now be clearly seen. The positive surface tension solutions deviate from the the zero-surface-tension solution, with the smaller the surface tension the larger the deviation.

What is the manifestation of the daughter singularity at times beyond $t_{d}$ ? CH99b have attempted to study this by evolving the interface (for $S=10^{-8}$ ) with accuracy for as long as possible. The initial evolution is computed in 60-digit arithmetic. Shortly after $t=t_{d}$, the precision is switched to double (the Nyquist frequency had approached $10^{-15}$ ), and the number of points was thereafter doubled whenever the spectrum approached under-resolution. The simulations shown in Fig. 21 ended with $N=32768$ and $\Delta t=10^{-7}$. This figure shows the interface in the tip region at $t=0.0502$. While the interface is seemingly nondescript, its maximum curvature curvature is 10 times its value at $t=0.049$, and the "singular region" has continued to spread in time. The small indentations are associated with these high curvatures, are very hard to resolve, and are a signature of the daughter singularity.

Given the difficulty of computing over long times with such small surface tensions, CH99b study for larger surface tensions the impact of daughter singularity on the physical domain. Fig. 22 shows a close-up of the evolution of the interface near one tip for the two surface tensions $S=10^{-4}$ and $S=10^{-5}$. These simulations now use the SSD formulation with $R=1$ (equal-arclength method) [81] (at these larger surface tensions the time-step constraints for the conformal mapping approach are too severe). The symmetric indentations arising from the daughter singularity have now lead to a bulged bubble that is spreading outwards. As it spreads, this bubble becomes susceptible to noise induced tip-splitting, and a fingering process begins on top of it (see [46]). These simulations suggest that surface tension sets a length scale for the bubble and finger widths. According to the asymptotic theory, this length-scale is related to the minimum distance of the complex singularity to the unit disk. 


\section{Boundary Integral Methods in Microstructure Evolution}

In this section, we present a review of recent applications of boundary integral methods to study microstructure evolution in solid-state diffusional phase transformations in 2 space dimensions. Diffusional phase transformations occur when the temperature of a uniform mixture of materials is lowered into a regime where the mixture becomes unstable. The system responds by nucleating second-phase precipitates surrounded by a nearly uniform matrix. See Fig. 23 for a sketch of a typical model domain.

The precipitates evolve via the long-range diffusion of matter among the distinct crystal phases until equilibrium is re-established or diffusion is stopped by further lowering of temperature. Many important structural materials such as steels, nickel and aluminum alloys are produced in this way. Microstructure, the detailed arrangement of distinct constituents on the microscopic level, is a critical variable that sets the macroscopic stiffness, strength and toughness of an alloy. Because of the industrial importance of diffusional phase transformations, there have been many research studies to model and characterize the transformation process.

One of the central assumptions of mathematical models of diffusional phase transformations is that the system evolves so as to decrease the total energy. This energy consists of an interfacial part, associated with the precipitate/matrix interfaces, and a bulk part due to the elasticity of the constituent materials. As we will see below, simulating the evolution process involves solving both a harmonic and a biharmonic-like equation for the (quasi-steady) diffusion and elastic fields respectively. The motion of interfaces is strongly mediated by surface tension between the different phases. Consequently, many of the ideas and methods developed in the fluid dynamic context may be applied directly in this materials science context.

In the absence of the elastic stress, interfacial area is reduced by the diffusion of matter from regions of high interfacial curvature to regions of low curvature. This results in the growth of large precipitates at the expense of small ones and is known as Ostwald ripening (coarsening) [141]. This coarsening process may severely degrade the properties of the alloy. In the early 1960s, an asymptotic theory was developed by Lifshitz \& Slyosov [117] and Wagner [205] (LSW) to predict the temporal power law of precipitate growth and a scaling behavior of the distribution of the droplet radius in the long time limit. In the LSW theory, the average precipitate radius $\left\langle R>\sim t^{1 / 3}\right.$ at long times. The LSW theory has two major restrictions, however. First, precipitates are assumed to be circular (spherical in 3-d) and second, the theory is valid only in the zero precipitate volume fraction limit. Extending the results of LSW to more realistic physical situations has been a subject of intense research interest. See the reviews by Voorhees [201, 202] and Johnson \& Voorhees [92] for a collection of recent references.

In this section, we discuss recent research (using the boundary integral numerical method) that has been performed to investigate diffusional evolution in systems in which the precipitate/matrix boundaries are unconstrained, the precipitate volume fraction may be finite and elastic effects may be considered. Because of space limitations, we will focus on only a few works which we consider to be representative of state-of-the-art research in this field.

We note that because the bulk elastic energy does not necessarily favor large precipitates over smaller ones, the coarsening process is affected most significantly by elastic stress. This is also confirmed experimentally as transitions from spherical to cuboidal to plate-shaped precipitates, alignment of precipitates along specific crystallographic directions and merging and splitting of precipitates are all observed [6, 38, 122, 130, 130, 193, 217]. In fact, precipitate splitting can actually lead to a decrease in average precipitate size over time. Thus, one of the goals of this line of research is to use elastic stress to control the evolution process so as to achieve desirable

microstructures. Consequently, here we will focus primarily on works which consider the effect of 
elasticity on the diffusional evolution.

\subsection{Historical Perspective}

The numerical study of coarsening using the boundary integral method with arbitrarily-shaped precipitates began with a study of purely quasi-steady diffusional evolution (i.e. no elastic stress) by McFadden, Voorhees, Boisvert \& Meiron [126, 203]. Since then, other boundary integral methods for quasi-steady diffusional evolution, in the absence of elastic stress, have been developed by Imaeda \& Kawasaki [90], Yokoyama \& Sekerka [216], Zhu, Chen \& Hou [219], and Akaiwa \& Meiron [1] among others.

The study of elastic effects in diffusional phase transformations using the boundary integral method (with arbitrary precipitate shapes) began with the work of Voorhees, McFadden \& Johnson (VMJ92) [204] who studied the evolution towards equilibrium of isolated precipitates in a cubic anisotropic homogeneous elastic medium. That is, the elastic constants of the precipitate and matrix are identical. More extensive simulations of microstructure evolution in homogeneous elastic media were later performed by Su \& Voorhees [182, 183] and Thornton, Akaiwa \& Voorhees (TAV99) [190]. In addition, Thompson, Su \& Voorhees (TSV94) [188] implemented a novel boundary integral method to calculate equilibrium precipitate shapes and their stability, in homogeneous media, without resorting to evolution.

The study of elastic inhomogeneity in diffusional phase transformations began with the work of Jou, Leo \& Lowengrub (JLL97) [93] who studied the evolution of precipitate/matrix systems in isotropic media. Boundary integral simulations in anisotropic, inhomogeneous elastic media were first performed by Schmidt \& Gross [168] and Schmidt, Mueller \& Gross [169]. These authors studied equilibrium precipitate shapes and their stability using a method analogous to that used by TSV94 in the homogeneous case (i.e. no time evolution). Later, Leo, Lowengrub, \& Nie (LLN00) developed a boundary integral method to study diffusional evolution in anisotropic, inhomogeneous media.

Before we turn to a discussion of specific work, we comment that microstructure evolution in diffusional phase transformations has also been studied using other numerical approaches, in both 2 and 3 dimensions, such as phase-field modeling (e.g. see [207, 208, 206, 99, 114, 115, 110, 125, 140, 162, 186] among others) and discrete atom methods (e.g. see [109, 108, 107]). Further, in 3 space dimensions, there has been very recent research on diffusional evolution in homogeneous and inhomogeneous elastic media by Thompson \& Voorhees [187] and Mueller \& Gross [133], respectively, using boundary integral methods.

\subsection{The Model}

The model of microstructural evolution we review here consists of diffusionally growing, arbitrarily shaped precipitates $\Omega^{i}$, where $i=1, \ldots p$ and $p$ is the total number of precipitates, in an elastically stressed matrix $\Omega^{M}$. The matrix domain $\Omega^{M}$ may be finite, infinite or periodically arranged in the plane with the latter two being the most popular choices. We will consider infinite $\Omega^{M}$ and we refer the reader to $[72,133,168,169]$ for discussions of the periodic cases. Further, the equations we present are non-dimensional and we use the notation and non-dimensionalization of LLN00.

Diffusion of a composition field $c$ is assumed to occur in the matrix phase only and is taken to be quasi-steady:

$$
\Delta c=0 \quad \text { in } \quad \Omega^{M} .
$$


The precipitate/matrix interfaces, $\Gamma_{i}$, are assumed to be coherent, and upon which a generalized Gibbs-Thomson boundary condition is given:

$$
c=c_{\Gamma} \equiv\left(\tau+\tau^{\prime \prime}\right) \kappa+Z g^{e l}, \quad \text { on } \quad \Gamma_{i}
$$

where $\tau=\tau(\theta)$ is the anisotropic surface tension and $\theta$ is the angle which the tangent of the interface makes with the $x$-axis (appropriate for 2 -d). We take $\tau+\tau^{\prime \prime}>0$. Further, $\kappa$ is the mean curvature (surface energy density), $g^{e l}$ is the elasticity energy density which is given below in Eq. (60). The constant $Z$ is a measure of the relative contribution of the elastic and surface energies and scales with a characteristic size of the system such as the average precipitate radius.

The generalized Gibbs-Thomson boundary condition (50) for the composition field is analogous to the Laplace-Young boundary condition for the pressure field in the fluid dynamics context. In the solid/solid context, the Gibbs-Thomson boundary condition was derived by Larché \& Cahn [106], Johnson \& Alexander [91] and Leo \& Sekerka [113] among others (see also [78]). Roughly speaking, this boundary condition reflects the idea that changing the shape of a precipitate changes both the surface energy and the elastic energy of the system.

We will also allow a finite mass flux into the system through the far-field condition

$$
-J=\lim _{R_{\infty} \rightarrow \infty} \frac{1}{2 \pi} \int_{\Gamma_{\infty}} \nabla c \cdot \mathbf{n} d s
$$

where $J$ is the total mass flux into the system and $\Gamma_{\infty}$ is a circular far-field boundary whose radius is $R_{\infty}$ and $\mathbf{n}$ is the outward pointing normal vector. If $J>0$, the total area of precipitates increases with time (growth). If $J=0$, then the total area remains fixed (equilibration or coarsening).

The normal velocity $V$ of each precipitate-matrix interface $\Gamma_{i}$ is computed through a flux balance at the interface,

$$
V=\left.\nabla c \cdot \mathbf{n}\right|_{\Gamma}
$$

where $\mathbf{n}$ is the normal vector pointing into $\Omega^{m}$.

We note that an analogy may be made between the diffusion problem here (with $Z=0$ ) and the Hele-Shaw problem described in section 3. In the Hele-Shaw problem, the pressure $p$ is the harmonic field, the velocity is proportional to $\nabla p \cdot \mathbf{n}$ through Darcy's law. Consequently, the surface tension enters the both systems in exactly the same way. Therefore, the difficulties associated with tracking interfaces in a Hele-Shaw cell also arise in this materials science context.

Finally, it remains to determine the elastic energy density $g^{e l}$. In order to calculate $g^{e l}$, the elastic fields must be computed on each interface $\Gamma_{i}$. The elastic fields arise because of misfit strains between the precipitate and matrix phases as well as far-field applied strains. Following the notation used in [112], misfit is taken into account through the constitutive relations between the stress $\Sigma$ and the strain $\mathcal{E}$. In each precipitate,

$$
\Sigma^{P}=C^{P}\left(\mathcal{E}^{P}-\mathcal{E}^{T}\right)
$$

where $\mathcal{E}^{T}$ denotes the misfit strain, while in the matrix,

$$
\Sigma^{M}=\mathcal{C}^{M} \mathcal{E}^{M} .
$$

In the remainder of this section, we denote the precipitate and matrix phases by the superscripts $P$ and $M$ respectively. Although the precipitates need not have the same elastic constants, we will 
make that assumption here for simplicity. In Eqs. (53) and (54), $\mathcal{C}$ denotes the orthotropic stiffness tensor, which using compact notation in 2 dimensions, is given by

$$
C^{\chi}=\left(\begin{array}{ccc}
c_{11}^{\chi} & c_{12}^{\chi} & 0 \\
c_{12}^{\chi} & c_{22}^{\chi} & 0 \\
0 & 0 & c_{66}^{\chi}
\end{array}\right)
$$

where $\chi=P, M$. For the case of a cubic material $\chi, c_{11}^{\chi}=c_{22}^{\chi}$ while for an isotropic material, $c_{11}^{\chi}=c_{22}^{\chi}$ and $c_{66}^{\chi}=\left(c_{22}^{\chi}-c_{11}^{\chi}\right) / 2$. In compact notation, the stress and strain are given by

$$
\mathcal{E}^{\chi}=\left(\begin{array}{c}
\mathcal{E}_{11}^{\chi} \\
\mathcal{E}_{22}^{\chi} \\
2 \mathcal{E}_{12}^{\chi}
\end{array}\right) \quad \text { and } \quad \Sigma^{\chi}=\left(\begin{array}{c}
\Sigma_{11}^{\chi} \\
\Sigma_{22}^{\chi} \\
\Sigma_{12}^{\chi}
\end{array}\right)
$$

where $\mathcal{E}_{i j}^{\chi}=\frac{1}{2}\left(u_{i, j}^{\chi}+u_{j, i}^{\chi}\right), u_{i, j}^{\chi}=\partial u_{i} / \partial x_{j}$ and $u_{j}^{\chi}$ are the displacement fields. The quasi-steady equations for the elastic fields are

$$
\Sigma_{l j, j}^{\chi}=0 \quad \text { in } \Omega^{\chi=M, P}, \quad l=1,2
$$

where $\Omega^{P}=\cup_{i} \Omega^{i}$ and the Einstein summation convention is used. We assume that the two-phase interface $\Gamma_{i}$ is coherent, and so the boundary conditions are given by the continuity of displacement,

$$
\left.\mathbf{u}\right|_{P}=\left.\mathbf{u}\right|_{M}, \quad \text { on } \quad \Gamma_{i}
$$

and traction,

$$
\left.t_{l}\right|_{P}=\Sigma_{l j}^{P} \mathbf{n}_{j}=\Sigma_{l j}^{M} \mathbf{n}_{j}=\left.t_{l}\right|_{M}, \quad \text { on } \quad \Gamma_{i}, \quad l=1,2 .
$$

Finally we take conditions

$$
\begin{aligned}
\lim _{r \rightarrow \infty} \mathcal{E}^{M} & =\mathcal{E}^{0} \\
\mathbf{u}^{P} & <\infty \text { in any finite ball around the origin, }
\end{aligned}
$$

where $\mathcal{E}^{0}$ is an applied far-field strain. Once the elasticity problem is solved, $g^{e l}$ is given by

$$
g^{e l}=\frac{1}{2} \Sigma^{P}:\left(\mathcal{E}^{P}-\mathcal{E}^{T}\right)-\frac{1}{2} \Sigma^{M}: \mathcal{E}^{M}+\Sigma^{M}:\left(\mathcal{E}^{M}-\mathcal{E}^{P}\right) \quad \text { on } \quad \Gamma_{i},
$$

where $\mathbf{a}: \mathbf{b} \equiv a_{i j} b_{i j}$. Lastly, we note that the diffusion/elasticity system evolves to lower the sum of the surface $W_{s}$ and elastic $W_{e l}$ energies:

$$
\begin{aligned}
W_{t o t} & =W_{s}+W_{e l} \\
W_{s} & =\int_{\cup_{i} \Gamma_{i}} \tau(\theta) d s, \text { and } W_{e l}=\sum_{\chi=M, P} \int_{\Omega \chi} \Sigma_{i j}^{\chi} \tilde{\mathcal{E}}_{i j}^{\chi} d A,
\end{aligned}
$$

where

$$
\tilde{\mathcal{E}}^{\chi}=\left\{\begin{array}{l}
\mathcal{E}^{P}-\mathcal{E}^{T} \quad \text { for } \chi=P \\
\mathcal{E}^{M} \text { for } \chi=M
\end{array}\right.
$$

In the presence of non-zero applied stress, $W_{e l}$ is infinite and an appropriate finite part must be taken. We refer the reader to [93] for details. A calculation shows that

$$
\dot{W}_{t o t}=\frac{d}{d t}\left(W_{s}+W_{e l}\right)=\int_{\Gamma}\left(\tau \kappa+Z g^{e l}\right) V d s=-\int_{\Omega^{M}}|\nabla c|^{2} d A .
$$




\subsection{Solving the Model}

The solution of the diffusional phase transformation system involves three main components: (i) the solution of the elasticity system (57)-(60) to determine $g^{e l}$, (ii) the solution of the diffusion system (49)-(51) to determine $V$ and (iii) the tracking of the precipitate/matrix interfaces. In this section, we discuss each of these components separately.

\subsubsection{The Diffusion System and Interface Tracking}

The solution of the exterior Dirichlet problem is obtained using the very efficient approach developed by Greenbaum, Greengard \& McFadden (GGM93) [72]. In this approach, a boundary integral formulation of the problem, originally due to Mikhlin [129], is used. Let $\mu$ be a dipole distribution and introduce $p$ source terms $A_{1}, \ldots, A_{p}$. Then, write the composition $c(\mathbf{x})$ in the matrix as

$$
c(\mathbf{x})=\frac{1}{2 \pi} \int_{\cup \Gamma_{i}} \mu\left(s^{\prime}\right)\left[\frac{\partial}{\partial n\left(s^{\prime}\right)} \log \left|\mathbf{x}-\mathbf{x}\left(s^{\prime}\right)\right|+1\right] d s^{\prime}+\sum_{k=1}^{p} A_{k} \log \left|\mathbf{x}-S_{k}\right|
$$

where $n\left(s^{\prime}\right)$ is the unit normal at the integration point $\mathbf{x}\left(s^{\prime}\right), s^{\prime}$ is arclength along $\Gamma_{i}$ and $S_{k}$ is a point inside $\Gamma_{k}$. Letting $\mathbf{x} \rightarrow \mathbf{x}(s) \in \Gamma_{j}$ leads to a set of modified $2^{\text {nd }}$-kind Fredholm boundary integral equations:

$$
\left(\tau+\tau^{\prime \prime}\right) \kappa(s)+Z g^{e l}=-\frac{\mu(s)}{2}+\int_{\cup \Gamma_{i}} \mu\left(s^{\prime}\right)\left[\frac{\partial}{\partial n\left(s^{\prime}\right)} \log \left|\mathbf{x}(s)-\mathbf{x}\left(s^{\prime}\right)\right|+1\right] d s^{\prime}+\sum_{k=1}^{p} A_{k} \log \left|\mathbf{x}(s)-S_{k}\right|(65)
$$

To determine the $A_{k}$, Eq. (65) is appended with

$$
J=\sum_{k=1}^{p} A_{k}, \quad \text { and } \quad 0=\int_{\Gamma_{j}} \mu\left(s^{\prime}\right) d s^{\prime}, \quad j=1, \ldots, p-1
$$

This system is invertible [129] and the modification to the usual $2^{\text {nd }}$-kind structure is due to the sources $A_{k}$. GGM92 developed an efficient preconditioner by considering the reduced system of Eqs. (65), without the integral operator, and Eq. (64). This reduced linear $p \times p$ system may be solved by Gauss elimination. This can be interpreted [93] as a type of SSD of the integral equations in the spirit of the SSD described earlier in the fluid dynamics context. SSD's for integral equations are discussed in more detail in the following section where the inhomogeneous elasticity integral equations are presented.

The implementation used by GGM93 to solve Eqs. (64)-(65)is highly accurate and efficient. The integrals are discretized with spectral accuracy using the trapezoid rule and the fast multipole method (FMM) [31, 74] is used to evaluate the discrete sums in $O(N)$ work. The iterative method GMRES [163] is then used to solve the discrete matrix system. Thus, the methods of GGM make it possible to solve the exterior diffusion problem for many more precipitates than was previously computationally affordable. With this improvement, interface tracking then becomes the limiting computational step in the simulation of pure diffusional systems.

Using the dipole formulation of GGM93, the normal velocity is given by

$$
V_{i}(s)=\frac{1}{2 \pi} \int_{\cup \Gamma_{j}} \mu_{s^{\prime}} \frac{\partial}{\partial s} \log \left|\mathbf{x}(s)-\mathbf{x}\left(s^{\prime}\right)\right| d s^{\prime}+\sum_{k=1}^{p} A_{k} \frac{\left(x_{1}(s)-x_{1 k}^{s}\right) x_{2, s}-\left(x_{2}(s)-x_{2 k}^{s}\right) x_{1, s}}{\left(x_{1}(s)-x_{1 k}^{s}\right)^{2}+\left(x_{2}(s)-x_{2 k}^{s}\right)^{2}},(67)
$$

where the integral is interpreted in the principal value sense. A spectrally accurate discretization of this integral is obtained using the alternate point trapezoidal rule analogous to that given in Eq. (36). Because of the third-order time-step constraint due to the surface tension (e.g. see Eq. (41)), 
interface tracking remained a problem for these systems until the recent work of HLS94. Their SSD applies directly to the diffusional system since

$$
V \sim \frac{1}{2} \mathcal{H}\left[\left(\tau+\tau^{\prime \prime}\right) \kappa\right]_{, s},
$$

at small spatial scales $[81,82,93,112]$ since the surface energy dominates the elastic energy at these scales. If one writes $\tau+\tau^{\prime \prime}=1+\left(\tau_{0}+\tau_{0}^{\prime \prime}\right)$, then one can follow the approach outlined previously for the Hele-Shaw case [81] and rewrite the interface evolution equations $\dot{\mathbf{X}}=V \mathbf{n}+T \mathbf{s}$ using the tangent angle $\theta$ and equal arclength tangential velocity $T$. Note that the equations are exactly as in the Hele-Shaw case (Eqs. 42) except that the definition of $N$ now includes the anisotropic surface energy. Except for the anisotropic surface energy contribution, the remainder terms $N$ are lower order at small spatial scales. Although the surface energy term $\mathcal{H}\left[\left(\tau_{0}+\tau_{0}^{\prime \prime}\right) \theta_{i, \alpha \alpha}\right]_{, \alpha}$ is of the same order as the term $\mathcal{H}\left(\theta_{i, \alpha \alpha \alpha}\right)$, it was found by Almgren, Dai \& Hakim [4] and LLN00 that the splitting in Eq. (42) is effective in removing the stiffness numerically provided that $\tau_{0}+\tau_{0}^{\prime \prime}$ is smooth and not too close to 1 .

At this point, we can discuss simulations of purely diffusional evolution in the absence of elastic stress $(Z=0)$. In metallic alloy systems, this corresponds to simulating systems of very small precipitates where the surface energy dominates the elastic energy. The current state-of-the-art in solving the pure diffusional evolution problem is the work of Akaiwa \& Meiron (AM96) [1]. AM96 combined the modified dipole approach of GGM93 together with the non-stiff time stepping of HLS94 to study the coarsening behavior of large precipitate/matrix systems with isotropic surface tension $\left(\tau_{0}=0\right)$. AM96 performed simulations containing over 5000 precipitates initially. Precipitates are removed if their area decreases below a certain tolerance. We note that the effect of removing precipitates was analyzed in [93] (elasticity was also considered). To make such large computations feasible, AM96 also took into account the effectively finite interaction distance of the diffusion field. For instance, particle-particle diffusional interactions may be neglected with only small relative errors if the particles are well enough separated [1]. To take advantage of this fact, AM96 divided the computational unit cell into sub-cells each containing 50-150 precipitates. Inside each sub-cell, the full diffusion field is computed. The influence of particles outside each sub-cell is restricted to only involve those lying within a distance of 6-7 times the average precipitate radius $\bar{R}$ from the sub-cell. This was found to give at most a $1 \%$ error in the diffusion field [1] and significantly reduces the computational cost.

The simulations of AM96 are impressive. For the diffusion systems they considered, the FMM is over 2, 500 times faster than using direct summation [72] and the original method of MVBM92. Since these integral equations must be solved at each time step, this is an enormous savings. Further, an additional factor of at least 1,000 in speed-up is gained by using the time stepping of HLS94 over that of a standard explicit method.

In Fig. 24, two snapshots of a typical simulation are shown at the very late stages of coarsening. In this simulation, the precipitate area fraction is 0.5 and periodic boundary conditions are applied to the unit cell. In Fig. 24(a), there are approximately 130 precipitates remaining while in Fig. 24(b) there are only approximately 70 precipitates left. Note that there is no discernible alignment of precipitates. Further, as the system coarsens, the typical shape of a precipitate shows significant deviation from a circle.

By using such large systems, AM96 were able to analyze the statistics. Their simulations agree with the classical Lifshitz-Slyozov-Wagner (LSW) theory $[117,205]$ in which the average precipitate radius $\langle R\rangle$ is predicted to scale as $\langle R\rangle \sim t^{1 / 3}$ at large times $t$. As seen in Fig. 24, AM96 find the persistence of non-circular precipitate shapes. Interestingly, although the particles are not circular, certain statistics, such as the particle size distribution functions, are found to be insensitive 
to the non-circular particle shapes at even at moderate volume fractions [1]. At volume fractions greater than 0.5 , the simulations become more difficult as the resulting small inter-particle spacings necessitate the use of adaptive refinement in space to accurately discretize the dipole and velocity integrals as well as adaptive refinement in time to accurately track the interfaces. This remains to be done in the future.

\subsubsection{The Homogeneous Elasticity System}

In homogeneous elastic media, the derivatives of the displacement field due to a misfitting precipitate may be evaluated directly from the Green's function tensor via the boundary integral:

$$
u_{j, k}(\mathbf{x})=\left(C_{i j 11}+\epsilon_{r} C_{i j 22}\right) \int_{\Gamma} g_{i j, k}\left(\mathbf{x}, \mathbf{x}^{\prime}\right) n_{l}^{\prime} d s^{\prime},
$$

where $\mathbf{x}$ is either in the matrix or precipitate and

$$
C_{i j k l}=2 c_{66} \delta_{i k} \delta_{j l}+c_{12} \delta_{i j} \delta_{k l}-\left(c_{11}-c_{12}-2 c_{66}\right) \delta_{i j k l},
$$

for cubic systems; here we do not use compact notation. The misfit tensor $\mathcal{E}^{T}$ is assumed to take the form

$$
\mathcal{E}^{T}=\left(\begin{array}{cc}
1 & 0 \\
0 & \epsilon_{r}
\end{array}\right)
$$

where $\epsilon_{r}$ is a constant. In two space dimensions, one may find an effectively analytic form for $g_{i j, k}$ that depends on the anisotropy ratio $A=2 c_{66} /\left(c_{11}-c_{12}\right)$. Taking the limit as $x \rightarrow x(s) \in \Gamma$, the result involves a rather complicated principal value integral whose exact form we do not present here; we instead refer the reader to the $[204,188,182,183]$. We note that $g^{\text {el }}$ is easily constructed once $u_{j, k}$ has been obtained (also see [204] for example).

The current state-of-the-art in simulating diffusional evolution in homogeneous, anisotropic elastic media is the recent work of Thornton, Akaiwa \& Voorhees (TAV99) [190, 189, 191] in which the coarsening of large cubic systems is studied. In metallic alloys, such a system can be considered as a model for nickel-aluminum alloys since in those alloys, the precipitates and matrix both have face-centered-cubic lattices with elastic constants typically differing by less than $10 \%$ [122]. TAV99 combined the improvements in interface tracking due to HLS94 and in the diffusion solver due to AM96 and GGM93. In addition, TAV99 developed a fast multipole method to evaluate the elasticity integral in Eq. (69). With these improvements, TAV99 achieved an enormous gain in computational efficiency and are now able to analyze the statistics of microstructure evolution in homogeneous elastic media.

In preliminary work, TAV99 have performed simulations involving over 4,000 precipitates initially. As in AM96, precipitates are removed when their area reaches a certain lower tolerance. See Fig. 25 for results with $Z=2.0,4.1$ and 6.1 and with isotropic surface tension and dilatational misfits. The value of $Z$ is allowed to vary dynamically through an average precipitate radius. Thus, as precipitates coarsen and grow larger, $Z$ increases correspondingly. The initial volume fraction of precipitates is 0.1 and simulations are started with $Z=1$. TAV99 find that the morphological evolution is significantly different in the presence of elastic stress. Unlike the purely diffusional case and consistent with experimental results, large-scale alignment of particles is seen in the $<100>$ and $<010>$ directions during the evolution process. In addition, there is significant shape dependence as nearly circular precipitates are seen at small $Z=2.0$ while at $Z=4.1$ and $Z=6.1$, precipitates are squarish and rectangular, respectively. As in AM96, certain features of the process are insensitive to the shape variation and work is continuing to determine the asymptotic rate of increase of $\langle R>$ in time. 


\subsubsection{The Inhomogeneous Elasticity System}

While the elastic constants of the nickel-aluminum precipitates and a nickel matrix may differ by only $10 \%$, there are other nickel-based alloys in which the elastic constants may differ by more than 50\%. Examples of such systems include nickel-silicon and nickel-gallium precipitates in a nickel matrix. It therefore is important to be able to account for elastic inhomogeneity. Indeed, the results of many investigations now indicate that even small elastic inhomogeneities may significantly impact precipitate evolution (e.g. [93, 112, 168, 169, 140, 114, 115, 162]).

The inhomogeneous elasticity system (57)-(60) is much more difficult to solve than the homogeneous system. Unlike the homogeneous case, integral equations must now be solved in order to obtain the inhomogeneous elastic fields. The elasticity system (57)-(60) may be reformulated in terms of boundary integrals as follows. Let $u_{i}(s)$ and $t_{i}(s)$ be the components of displacement and traction on the boundary, respectively. One may then use a direct approach, involving both elastic monopoles and dipoles, to obtain a system of four coupled integral equations for the displacement and traction components on each interface $\Gamma$ :

$$
\begin{gathered}
\frac{u_{j}(s)}{2}+\int_{\Gamma} u_{k}\left(s^{\prime}\right) T_{k j}^{P}\left(\mathbf{x}(s), \mathbf{x}\left(s^{\prime}\right)\right) d s^{\prime}-\int_{\Gamma} t_{k}\left(s^{\prime}\right) U_{k j}^{P}\left(\mathbf{x}(s), \mathbf{x}\left(s^{\prime}\right)\right) d s^{\prime} \\
=\int_{\Gamma} t_{k}^{T}\left(s^{\prime}\right) U_{k j}^{P}\left(z(s), z\left(s^{\prime}\right)\right) d s^{\prime} \\
-\frac{u_{j}(s)}{2}+\int_{\Gamma} u_{k}\left(s^{\prime}\right) T_{k j}^{M}\left(\mathbf{x}(s), \mathbf{x}\left(s^{\prime}\right)\right) d s^{\prime}-\int_{\Gamma} t_{k}\left(s^{\prime}\right) U_{k j}^{M}\left(\mathbf{x}(s), \mathbf{x}\left(s^{\prime}\right)\right) d s^{\prime} \\
=-\frac{u_{j}^{A}(\mathbf{x}(s))}{2} \int_{\Gamma} u_{k}^{A}\left(s^{\prime}\right) T_{k j}^{M}\left(\mathbf{x}(s), \mathbf{x}\left(s^{\prime}\right)\right) d s^{\prime}-\int_{\Gamma} t_{k}^{A}\left(s^{\prime}\right) U_{k j}^{P}\left(\mathbf{x}(s), \mathbf{x}\left(s^{\prime}\right)\right) d s^{\prime}(72)
\end{gathered}
$$

where $\Gamma=\cup_{i} \Gamma_{i}$ and $U^{P}\left(\mathbf{x}, \mathbf{x}^{\prime}\right), U^{M}\left(\mathbf{x}, \mathbf{x}^{\prime}\right)$ are the fundamental solution tensors in the precipitate and matrix domains (considered separately) that generate displacement vectors $u_{j}(\mathbf{x})$ arising from an isolated point source located at $\mathbf{x}^{\prime}$ in the precipitate and the matrix respectively. In Eq. (72), $T^{P}$ and $T^{M}$ are the analogous traction tensor fields. We do not give the exact form of $U^{\chi}$ and $T^{\chi}$ here and instead we refer the reader to JLL97 for the isotropic case and LLN00 for the orthotropic case (see also $[153,154,168,169]$ ). The $U^{\chi}$ contains both smooth and logarithmic functions while the $T^{\chi}$ contains both smooth functions and functions with Cauchy type singularities; the $T^{\chi}$ integrals are interpreted in the principle value sense. Finally in Eqs. (71) and (72), $\mathbf{t}^{T}$ is the traction vector due to the misfit strain in the precipitate $\mathbf{t}^{T}=C \mathcal{E}^{T}$ and $\mathbf{u}^{A}$ and $\mathbf{t}^{A}$ are the displacement and traction vectors of a constant applied stress field $\Sigma^{A}$.

\section{The Small Scale Decomposition for Integral Equations}

Although the system (71)-(72) is a Fredholm equation of mixed type with smooth, logarithmic and Cauchy type kernels, it was shown by JLL97, in the isotropic case, and by LLN00, in the anisotropic case, that it may be transformed directly to a 2nd kind Fredholm system with smooth kernels. The transformation relies on an analysis of the equations at small spatial scales and is in spirit like the SSD in the fluid dynamic context. Classical Fredholm theory [152] may be used to guarantee the existence and uniqueness of solutions since the displacement is uniquely specified at infinity $\left(\mathbf{u}^{A}\right)$.

If the Eqs. (71)-(72) are discretized directly, the condition numbers increase at least linearly with $N$ (the total number of points) due to the logarithmic kernels. The condition number also increases slowly as the number of precipitates $p$ increases. To overcome this ill-conditioning, JLL97 and LLN00 performed a small scale analysis of the equations to determine the dominant terms at 
small scales in the spirit of HLS94. For example, suppose a system of integral equations may be written as

$$
\mathcal{A}[\mathbf{u}, \mathbf{t}]=\mathbf{f}
$$

and suppose that $\mathcal{A} \sim \mathcal{L}$ at high wave-numbers while the remainder involves integration against smooth kernels. Then one may write

$$
\mathcal{A}=\mathcal{L}+\mathcal{R} .
$$

If $\mathcal{L}$ is diagonalizable in Fourier space, and so is easily inverted using the FFT, one may re-write Eq. (73) as

$$
\left(I+\mathcal{L}^{-1} \mathcal{R}\right)[\mathbf{u}, \mathbf{t}]=\mathcal{L}^{-1} \mathbf{f},
$$

which is a $2^{\text {nd }}$-kind Fredholm equation. When discretized, Eq. (75) is well-conditioned [93]. See [219], for example, for an application to a 1st kind Fredholm equation for the Dirichlet problem.

\section{Application to the Inhomogeneous Elasticity Integral Equations}

In the elasticity case, the SSD reveals that the dominant operator at small scales is

$$
\mathcal{L}=\left(\mathcal{L}_{1,1,1}, \ldots, \mathcal{L}_{1,1, p}, \mathcal{L}_{1,2,1}, \ldots, \mathcal{L}_{1,2, p}, \mathcal{L}_{2,1}, \mathcal{L}_{2,2}\right),
$$

where the components of $\mathcal{L}$ are then given by

$$
\begin{aligned}
\mathcal{L}_{1, l, j}[\mathbf{u}, \mathbf{t}] & =\frac{1}{2} u_{l}-D_{1 l k}^{P} \mathcal{H}\left[u_{k}\right]-D_{2 l k}^{P} \mathcal{H}\left[\tilde{t}_{k}\right], \\
\mathcal{L}_{2, l}[\mathbf{u}, \mathbf{t}] & =\frac{1}{2} u_{l}-D_{1 l k}^{P} \mathcal{H}\left[u_{k}\right]-D_{2 l k}^{M} \mathcal{H}\left[\tilde{t}_{k}\right],
\end{aligned}
$$

and $\partial_{\alpha} \tilde{t}_{k}=L_{j} t_{k} / 2 \pi$ and $D_{1,2}^{P, M}$ depend only on the elastic constants (see [93, 112] for details). Note that the $u_{k}$ and $\tilde{t}_{k}$ that appear on the right hand sides of Eqs. (77) and (78) are only evaluated on $\Gamma_{j}$. In contrast, $\mathcal{A}$ involves evaluation of $u_{k}$ and $t_{k}$ on all the precipitate/matrix interfaces $\cup_{j} \Gamma_{j}$. Although $\mathcal{L}$ is a nonlocal operator, solving

$$
\mathcal{L}[\mathbf{u}, \mathbf{t}]=b
$$

only involves inverting a $4 \times 4$ matrix in Fourier space since $\mathcal{L}$ is diagonalized by the Fourier transform. This is much simpler than solving the full system. Further, if the precipitate/matrix boundaries are smooth, then the remainder operator $\mathcal{R}$ involves integration against smooth kernels and thus is a smoothing operator. Roughly speaking this means that as an operator, $\mathcal{R}$ has a rapidly decaying Fourier symbol [93]. This type of small scale decomposition for integral equations obviates the need to use Goursat functions to formulate $2^{\text {nd }}$-kind equations directly for the elasticity system [129, 73]. Typically, this SSD preconditioning reduces the iteration count by about a factor of about 5 over that for the unpreconditioned system [93, 112]. What remains of the original illconditioning is that $\widehat{\mathcal{L}}^{-1} \sim|k|$ for large wave-numbers $k$. Thus, numerical errors in $\mathbf{f}$ are amplified by the application of $\mathcal{L}^{-1}$. Numerical filtering $[81,104]$ is used to control this amplification.

We remark that Greengard \& Helsing (GH98) [73] have recently developed a fast algorithm for computing elastic fields in isotropic, inhomogeneous elastic media. Their algorithm is based on an indirect integral equation approach using complex potentials, due to Sherman [177], combined with the fast multipole method and adaptive Gauss-Legendre quadrature. In this approach, a single 2nd kind Fredholm equation for each interface is obtained directly for a Cauchy layer potential. This alone presents an enormous savings over the direct approach discussed above where four equations must be solved for each interface. However, the methods of GH98 currently only account for applied fields and not for precipitate misfit strains. Nevertheless, this approach is very promising for use in microstructure evolution and Helsing \& Lowengrub are currently studying the extension to the case of misfitting precipitates. 


\subsubsection{Interface Tracking in the Face of Anisotropy}

LLN00 observed that certain microscopic details of the numerical solution, such as $c_{\Gamma}$, may depend sensitively on the numerical time integration algorithms. This sensitive dependence arises when large gradients are present. Such gradients are typically associated with the formation of "corners" or regions of high curvature. Since corners naturally arise in equilibrium precipitate shapes in the presence of anisotropy, care must be taken to compute the solutions accurately. Time stepping methods based on an integrating factor approach, as in Eq. (47) tend to overdamp the numerical solution. This may cause oscillations to occur in $c_{\Gamma}$ and prevent attaining $\dot{W}_{\text {tot }}=0$ (computed from Eq. (63)) as equilibrium is approached. Macroscopic properties such as particle shapes and curvatures are less sensitive to the algorithms.

LLN00 developed accurate time discretizations by requiring that certain properties of the continuous equations be maintained on the discrete level. For example, in equilibrium, there is an exact balance between the linear and nonlinear terms in Fourier space:

$$
\frac{m(k)}{\left(L^{*}\right)^{3}} \hat{\theta}^{*}(k)=\hat{N}^{*}(k),
$$

where $*$ denotes the equilibrium value (recall Eq. (42)) and $m(k)=(2 \pi|k|)^{3}$. It is straightforward to see that integrating factor method in Eq. (47) violates this condition. The amount by which condition (79) is violated will be small if either $\hat{\theta}^{*}$ is exponentially small or $m \Delta t$ is small. If large gradients are present, $\hat{\theta}^{*}$ may be exponentially small only for rather large wave-numbers $k$ and hence very large $m$. For example, suppose exponential decay is observed for $|k|>k^{*}$, then the error is small only if $m\left(k^{*}\right) \Delta t<<1$. This is an accuracy restriction. If this is violated, then the error term may be significant even though the integrating factor method is formally second order accurate in time. Typically, $c_{\Gamma}$ and $\dot{W}_{\text {tot }}$ are sensitive to this error while interface position and curvature are not.

If $k^{*}$ is large, then the accuracy condition $m\left(k^{*}\right) \Delta t<<1$ may be very restrictive. By reducing the degree of damping at high wave-numbers, the accuracy restriction may be removed completely while the overall order of accuracy is maintained. Reducing the amount of damping does narrow the stability region of the numerical scheme, however. LLN considered three numerical schemes for which Eq. (79) is satisfied exactly on the numerical level. The scheme that is found to perform the best is given by

$$
\hat{\tilde{\theta}}^{n+1}=\frac{1}{1+m\left(I^{n+1}-I^{n-1}\right) e^{m\left(I^{n}-I^{n-1}\right)}} \hat{\tilde{\theta}}^{n-1}+2 \Delta t \frac{\hat{N}^{n}}{m\left(I^{n+1}-I^{n-1}\right)+e^{-m\left(I^{n}-I^{n-1}\right)}} .
$$

In this scheme, the linear term is damped exponentially while the nonlinear term is damped only algebraically.

\subsubsection{Results on Microstructure Evolution in Inhomogeneous, Isotropic Elastic Media}

The current state-of-the-art in simulating microstructure evolution in inhomogeneous, isotropic elastic media is the work of JLL97. In their approach, the interface tracking of HLS94 is combined together with the diffusion solver of GGM93 and with the elasticity solver described above. The method of JLL97 is spectrally accurate although a 25th order accurate Fourier filter is used to control numerical aliasing error (e.g. see HLS94).

JLL97 investigated both growth and coarsening of systems of precipitates with isotropic surface

tension. In the case of a shear misfit tensor (i.e. $\mathcal{E}_{11}^{T}=-\mathcal{E}_{22}^{T}, \mathcal{E}_{12}^{T}=0$ ), which introduces cubic anisotropy, growing shapes of single precipitates are seen to be dendritic while equilibrium shapes 
are squarish. See Fig. 26 for a sample of results. In (a) and (b), the numerical flux is $J=10$. In (a), the shear modulus of the precipitate is half of that of the matrix (soft precipitate) while the opposite is true in (b) (hard precipitate). Note that the dendrite arms are more slender for the soft precipitate than for the hard precipitate. In addition, wiggles form on the dendrite arms of the soft precipitate which indicates additional instability in the soft case relative to the hard. We also note that the observed dendritic structure in both cases qualitatively resembles that obtained using no elasticity $(Z=0)$ but anisotropic surface tension [4] (see Fig. 14). In Fig. 26(e), the corresponding case with isotropic surface tension and $Z=0$ is shown. Note the isotropic nature of the curve, as well as the occurrence of tip splitting, is suppressed by the elasticity. In Fig. 26(d), the convex curve corresponds to the equilibrium precipitate shape obtained by setting $J=0$ at $t=20$ for the soft precipitate (the non-convex shape shown in the figure).

In Fig. 27, a sample simulation is shown of 16 precipitates coarsening $(J=0)$ in inhomogeneous, isotropic elastic media. Precipitates are removed when their area drops below 0.1. Because of the isotropic surface tension and choice of misfit (tetragonal with $\mathcal{E}_{11}^{T} \neq \mathcal{E}_{22}^{T}>0, \mathcal{E}_{12}^{T}=0$ ), the precipitates develop roughly elliptical shapes. An overall classical coarsening behavior occurs as large precipitates grow at the expense of small ones. Further, over the time scales shown, there is no significant precipitate translation or alignment. Thus, it appears that alignment is a secondary process that occurs after shape changes and coarsening.

When precipitates are removed, the surface and elastic energies are continuous but the surface energy is kinked. In fact, it may be shown that $\dot{W}_{t o t} \sim\left(t_{c}-t\right)^{-2 / 3}$ [93]. This may be seen explicitly in Fig. 28 where $W$ and $\dot{W}$ are shown for the simulation in Fig. 27. As expected, $\dot{W}_{\text {tot }}$ drops sharply just before a precipitate is removed. Because precipitates are removed after their area drops below 0.1 , their size when they are actually removed varies. Thus, the drops in $\dot{W}_{\text {tot }}$ vary. Interestingly, the envelope of $\dot{W}_{\text {tot }}$ appears to be a continuous curve which suggests that the overall evolution is insensitive to precipitate removal.

\subsubsection{Results on Microstructure Evolution in Inhomogeneous, Anisotropic Elastic Media}

The current state-of-the-art in simulating microstructure evolution in inhomogeneous, orthotropic elastic media is the work of LLN00. In their method, LLN00 coupled the diffusion solver developed by GGM93 together with a solution of the orthotropic Rizzo-Shippy elastic integral equations with small scale preconditioning. Because no fast algorithm yet exists for evaluating the elasticity integral equations, an efficient parallel implementation of direct summation was used for both the diffusion and elastic systems.

LLN00 found that even small elastic inhomogeneities may have a strong effect on precipitate evolution. For instance, in systems where the elastic constants of the precipitates are smaller than those of the matrix, the precipitates move toward each other; The rate of approach depends on the amount of inhomogeneity. Anisotropic surface energy may either enhance or reduce this effect.

To illustrate this effect, consider the evolution of four types of precipitates in a nickel matrix: (i) homogeneous $(N i)$, (ii) nickel-silicon $\left(N i_{3} S i\right)$, (iii) nickel-aluminum $\left(N i_{3} A l\right)$, and (iv) nickel-gallium $\left(N i_{3} \mathrm{Ga}\right)$. The $\mathrm{Ni}_{3} \mathrm{Si}$ precipitates have larger elastic constants (hard) than the $\mathrm{Ni}$ matrix while both the $\mathrm{Ni}_{3} \mathrm{Al}$ and $\mathrm{Ni}_{3} \mathrm{Ga}$ precipitates have smaller constants (soft) than the matrix. Moreover, both the precipitates and matrix are cubic anisotropic with anisotropy ratio $A>1$. See LLN00 for details.

In Fig. 29, the evolution of two precipitates is shown for each of the systems (i)-(iv). The initial configuration in all cases consists of two unit circles separated by a distance of 1 unit. $Z=5$ for

all cases. For the homogeneous case and the case with $\mathrm{Ni}_{3} \mathrm{Si}$ particles, the final times correspond 
to when particle evolution essentially ceased. For the cases with $N i_{3} \mathrm{Al}$ and $\mathrm{Ni}_{3} \mathrm{Ga}$ particles, the final times correspond to when the interparticle spacing was too small to resolve the interfaces for the numerical parameters used.

We observe that the spacing between the hard $\mathrm{Ni}_{3} \mathrm{Si}$ particles is larger than the spacing between the homogeneous particles, while the spacing between the soft $N i_{3} \mathrm{Al}$ and $N i_{3} \mathrm{Ga}$ particles is smaller than the spacing between the homogeneous particles. This is consistent with simulations in isotropic media where that hard particles repel, while soft particles attract [93]. However, the situation is more complex with anisotropic elasticity. In the homogeneous and the $N i_{3} S i$ case, the particles move towards each other with very small velocities which tend to zero at a finite interparticle distance. In the $\mathrm{Ni}_{3} \mathrm{Ga}$ case, the interparticle attraction increases as the interparticle spacing decreases. This suggests the particles will merge. In the $N i_{3} A l$ case (which is nearly homogeneous), however, the interparticle attraction begins to decrease when the particles are very close to one another. More refined calculations are necessary to determine whether there is in fact a small non-zero interparticle spacing in this case. The difference between the $N i_{3} G a$ and $N i_{3} A l$ cases is also reflected in the particle shapes in the interparticle region; in the $N i_{3} \mathrm{Ga}$ case, the particles appear to curved towards each other while in the $N i_{3} \mathrm{Al}$ case the two particles have 'squared off' against each other.

Finally, we conclude this section by presenting some recent preliminary results, obtained by LLN00 [111], which indicate that an elastically-driven morphological instability may occur in the presence of applied fields. Inhomogeneity plays a crucial role in the instability as an analogous instability has not yet been observed in homogeneous systems [111]. Moreover, the instability apparently leads to precipitate splitting. This may be the source of splitting observed in experiments $[94,217]$ for example. A similar morphological instability has been observed in simulations using the discrete atom method [109, 108].

The elastic morphological instability may be seen in the following context. Consider the evolution of a single $\mathrm{Ni}_{3} \mathrm{Si}$ precipitate with applied shear and dilatational misfit. This is shown in Fig. 30(a) with $Z=5$ and isotropic surface tension. In this simulation, the initial condition is a circle. The interface is then shown at subsequent times near pinch-off. Observe that the interface forms two inter-penetrating fingers that grow towards one another strongly suggesting that the precipitate will split into two plate-like precipitates with long-axes oriented in the $x_{2}$-direction. There is a very slight amount of asymmetry in the plot due to numerical error and more refined simulations are currently being performed; convergence tests at earlier times confirm that the instability is physical and not numerical. For the purposes of comparison, Fig. 30(b) shows the evolution of the precipitate with $Z=3$. In this simulation, there is a hint of instability at small times although the precipitate boundary stabilizes and becomes elliptical. In Fig. 30(c), the evolution of a homogeneous precipitate is shown with $Z=5$ (solid squarish line) and $Z=10$ (dashed line). In the homogeneous case, the precipitate converges to a squarish equilibrium shape which is remarkably different from that seen in the inhomogeneous cases.

\section{Conclusion and Future Directions}

In this paper, we have presented a brief review of the application of boundary integral methods in two dimensions to multi-component fluid flows and multi-phase problems in materials science. In particular, we have focused on results from the recent development of methods which accurately and efficiently include surface tension. In fluid flows, we have examined the effects of surface tension on the Kelvin-Helmholtz and Rayleigh-Taylor instabilities, the generation of capillary waves on a free surface, and problems involving pattern generation and selection, and singularity formation, in 
Hele-Shaw flows. In materials science, we discussed microstructure evolution in diffusional phase transformations and the effects of the resulting competition between surface and elastic energies on the microstructure morphology.

The significant recent developments underlying these simulational studies are (i) the analyses of BHL93/BHL96 and BN98 which identify and correct instabilities generated by spatial discretization, and most especially (ii) the introduction of the SSD by HLS94/HLS97 to develop very efficient and accurate time discretization methods. Until these works, simulations were fraught with numerical instability and were greatly limited in accuracy and temporal evolution. The above works have provided the backbone of new classes of methods which have enabled the study of much more complex phenomena than was previously possible.

Many challenges remain for the future. These include the extension of the ideas presented here to more complicated flows such as those involving viscosity and/or elastic boundary forces (e.g. from simulations of heart function), as well as to axisymmetric and 3-d interface evolution. In axisymmetric flows, the SSD has recently been extended to the vortex sheet case by Nie [135], and to axisymmetric porous media flow by Ceniceros \& Si [37]. The development of accurate and efficient boundary integral methods for interface evolution in 3-d is a highly non-trivial problem and has been the subject of much recent research (see, for example [76, 20, 84, 83]). The development of efficient and accurate time-stepping algorithms in 3-d, analogous to those in 2-d based on the $\mathrm{SSD}$, is an outstanding problem. In the materials science context, where the time-step restrictions are $3^{r d}$-order, the development of efficient time integration methods is crucial. Although there have been three dimensional boundary integral simulations recently performed by Thompson \& Voorhees [187] for homogeneous systems and by Mueller \& Gross [133] for inhomogeneous systems, those works were limited by spatial and temporal resolution requirements.

\section{Acknowledgements}

The authors thank R. Almgren, N. Akaiwa, P.H. Leo, D. Meiron, Q. Nie, D. Thompson, K. Thornton and P. Voorhees who provided us with figures from their recent results. TYH acknowledges support from NSF grant DMS-9704976 and ARO grant DAAD19-99-1-0141 (ARO). JL acknowledges support from NSF grant DMS-9706931 and from the Minnesota Supercomputer Institute. MJS acknowledges support from NSF grant DMS-9707494 and DOE grant DE-FG02-88ER25053.

\section{References}

[1] N. Akaiwa and D.I. Meiron. Two-dimensional late-stage coarsening for nucleation and growth at high-area fractions. PHYSICAL REVIEW E, 54(1):R13-R16, 1996.

[2] R. Almgren. Singularity formation in Hele-Shaw bubbles. Physics of Fluids, 8(2):344-352, 1996.

[3] R. Almgren, A. Bertozzi, and M.P. Brenner. Stable and unstable singularities in the unforced Hele-Shaw cell. Physics of Fluids, 8(6):1356-1370, 1996.

[4] R. Almgren, W.-S. Dai, and V. Hakim. Scaling behavior in anisotropic Hele-Shaw flow. Physical Review Letters, 71(21):3461-3464, 1993.

[5] C. Anderson. A vortex method for flows with slight density variations. J. Comp. Phys., 61:417, 1985. 
[6] A.J. Ardell and R.B. Nicholson. On the modulated structure of aged Ni-Al alloys. Acta Metall., 14:1295, 1966.

[7] A. Arnéodo, Y. Couder, G. Grasseau, V. Hakim, and M. Rabaud. Uncovering the analytical Saffman-Taylor finger in unstable viscous fingering and diffusion-limited aggregation. Physical Review Letters, 63(9):984-987, 1989.

[8] U. Ascher, S. Ruuth, and B. Wetton. Implicit-explicit methods for time-dependent partial differential equations. SIAM J. Num. Anal., 32:797, 1995.

[9] D. H. Bailey. MPFUN: A portable high perfomance multiprecision package. Technical Report RNR-90-022, NASA Ames Research Center, 1990.

[10] G. Baker, D. Meiron, and S. Orszag. Boundary integral methods for axisymmetric and 3dimensional Rayleigh-Taylor instability problems. Physica D, 12:19-31, 1984.

[11] G. Baker and A. Nachbin. Stable methods for vortex sheet motion in the presence of surface tension. SIAM J. Sci. Comput., 19:1737-1766, 1998.

[12] G. Baker, M. Siegel, and S. Tanveer. A well-posed numerical method to track isolated conformal-map singularities in Hele-Shaw flow. Journal of Computational Physics, 120(2):348-364, 1995.

[13] G. R. Baker, D. I. Meiron, and S. A. Orszag. Vortex simulations of the Rayleigh-Taylor instability. Phys. Fluids, 23:1485-1490, 1980.

[14] G. R. Baker, D. I. Meiron, and S. A. Orszag. Generalized vortex methods for free-surface flow problems. J. Fluid Mech., 123:477-501, 1982.

[15] G.R. Baker and D.W. Moore. The rise and distortion of a two dimensional gas bubble in an inviscid fluid. J. Fluid Mech., 123:477, 1982.

[16] G.R. Baker and Q. Nie. The asymptotic motion of an accelerating, thick layer of inviscid fluid. Phys. Fluids, 10:101, 1998.

[17] G.R. Baker and M.J. Shelley. On the connection between thin vortex layers and vortex sheets. J. Fluid Mech., 215:161, 1990.

[18] J. Beale, T. Hou, and J. Lowengrub. Growth rates for the linear motion of fluid interfaces far from equilibrium. Comm. Pure Appl. Math., 46:1269, 1993.

[19] J. Beale, T. Hou, and J. Lowengrub. Convergence of a boundary integral method for water waves. SIAM J. Numer. Anal., 33:1797-1843, 1996.

[20] J. T. Beale. A convergent boundary integral method for 3-D water waves. preprint, submitted to Math. Comput., 1998.

[21] J.T. Beale, T.Y. Hou, and J.S. Lowengrub. On the well-posedness of two fluid interfacial flows with surface tension. In R. Caflisch and G. Papanicolaou, editors, Singularities in Fluids, Plasmas and Optics, Nato Adv. Sci. Inst. Ser. A, page 11. Kluwer, Amsterdam, 1993.

[22] J.T. Beale, T.Y. Hou, J.S. Lowengrub, and M.J. Shelley. Spatial and temporal stability issues for interfacial flows with surface tension. Math. Comput. Modelling, 20:1, 1994. 
[23] D. Bensimon, L.P. Kadanoff, S.D. Liang, B.I. Shraiman, and C. Tang. Viscous flows in 2 dimensions. Reviews of Modern Physics, 58(4):977-999, 1986.

[24] A.L. Bertozzi, M.P. Brenner, T.F. Dupont, and L.P. Kadanoff. Singularities and similarities in interface flows. In L. Sirovich, editor, Trends and Perspectives in Applied Mathematics, pages 155-208. Springer, 1994.

[25] A.L. Bertozzi and M. Pugh. The lubrication approximation for thin viscous films - regularity and long-time behavior of weak solutions. Communications on Pure and Applied Mathematics, 49(2):85-123, 1996.

[26] G. Birkhoff. Helmholtz and taylor instability. In Proc. Symp. Appl. Math., volume 13, pages 55-76. American Math. Society, Providence, R.I., 1962.

[27] J.M. Boulton-Stone and J.R. Blake. Gas bubbles bursting at a free surface. J. Fluid Mech., 254:437, 1993.

[28] J. Broeze, E. F. G. Van Daalen, and P. J. Zandbergen. A three-dimensional panel method for nonlinear free surface waves on vector computers. Comput. Mech., 13:12-28, 1993.

[29] R. Brower, D. Kessler, J. Koplik, and H. Levine. Geometrical models of interface evolution. Phys. Rev. A, 29:1335-1342, 1984.

[30] D. L. Brown, W. D. Henshaw, and D. J. Quinlan. Overture: An object oriented framework for solving partial differential equations. In Scientific Computing in Object-Oriented Parallel Environments. Springer Lecture Notes in Computer Science, 1343, 1997.

[31] J. Carrier, L. Greengard, and V. Rokhlin. A fast adaptive multipole algorithm. SIAM J. Sci. Stat. Comput., 9:669, 1988.

[32] L. Carrillo, F.X. Magdaleno, J. Casademunt, and J. Ortin. Experiments in a rotating HeleShaw cell. Physical Review E, 54(6):6260-6267, 1996.

[33] H. D. Ceniceros and T. Y. Hou. Convergence of a non-stiff boundary integral method for interfacial flows with surface tension. Math. Comput., 67:137-182, 1998.

[34] H. D. Ceniceros and T. Y. Hou. Dynamic generation of capillary waves. Phys. Fluids, 11(5):1042-1050, 1999.

[35] H. D. Ceniceros and T. Y. Hou. The singular perturbation of surface tension in Hele-Shaw flows. J. Fluid Mech, 409:251-272, 2000.

[36] H. D. Ceniceros, T.Y. Hou, and H. Si. Numerical study of Hele-Shaw flow with suction. Phys. Fluids, 11(9):2471-2486, 1999.

[37] H. D. Ceniceros and H. Si. Computation of axi-symmetric suction flow through porous media in the presence of surface tension. to appear, J. Comput. Phys., 2000.

[38] J. Conley, M.E. Fine, and J.R. Weertman. Effect of lattice disregistry variation on the late stage phase transformation behavior of precipitate in Ni-Al-Mo alloys. Acta Metall., 37:1251, 1989 .

[39] P. Constantin, T.F. Dupont, R.E. Goldstein, L.P. Kadanoff, M.J. Shelley, and S.M. Zhou. Droplet breakup in a model of the Hele-Shaw cell. Physical Review E, 47(6):4169-4181, 1993. 
[40] P. Constantin and L. Kadanoff. Dynamics of a complex interface. Physica D, 47:450-460, 1991.

[41] P. Constantin and M. Pugh. Global solutions for small data to the Hele-Shaw problem. Nonlinearity, 6:393-415, 1993.

[42] S. Cowley, G. Baker, and S. Tanveer. On the formation of Moore curvature singularities in vortex sheets. J. Fluid Mech., 378:233-267, 1999.

[43] W. Craig and C. Sulem. Numerical simulation of gravity waves. J. Comput. Phys., 108:73, 1993.

[44] G. D. Crapper. Non-linear capillary waves generated by steep gravity waves. J. Fluid Mech., 40:149-159, 1970.

[45] W.-S. Dai, L.P. Kadanoff, and S.M. Zhou. Interface dynamics and the motion of complex singularities. Physical Review A, 43(12):6672-6682, 1991.

[46] W.-S. Dai and M. J. Shelley. A numerical study of the effect of surface tension and noise on an expanding Hele-Shaw bubble. Phys. Fluids A, 5(9):2131-2146, 1993.

[47] M.R. Davidson. An integral equation for immiscible fluid displacement in a two-dimensional porous medium or Hele-Shaw cell. Journal of the Australian Mathematical Society Series B, $26: 14-30,1984$.

[48] M.R. Davidson. Numerical calculation of unstable immiscible fluid displacement in a twodimensional porous medium or Hele-Shaw cell. Journal of the Australian Mathematical Society Series B, 26:452-469, 1985.

[49] A.I Van de Vooren. A numerical investigation of the rolling up of vortex sheets. Proc. Roy. Soc. London Ser. A, 373:67, 1980.

[50] A.J. Degregoria and L.W. Schwartz. Finger breakup in Hele-Shaw cells. Physics of Fluids, 28(8):2313-2314, 1985.

[51] A.J. Degregoria and L.W. Schwartz. A boundary-integral method for 2-phase displacement in Hele-Shaw cells. Journal of Fluid Mechanics, 164:383-400, 1986.

[52] A.J. Degregoria and L.W. Schwartz. Saffman-Taylor finger width at low interfacial tension. Physical Review Letters, 58(17):1742-1744, 1987.

[53] J. W. Dold. An efficient surface-integral algorithm applied to unsteady gravity waves. J. Comput. Phys., 103:90-115, 1992.

[54] D.G. Dommermuth and D.K.P. Yue. A high order spectral method for the study of nonlinear gravity waves. J. Fluid Mech., 184:267, 1987.

[55] P. G. Drazin and W. H. Reid. Hydrodynamic stability. Cambridge monographs on mechanics and applied mathematics. Cambridge University Press, New York, 1981.

[56] J. Duchon and R. Robert. Évolution d'une interface par capillarité et diffusion de volume I. Existence locale en temps. Ann. Inst. Henri Poincaré, 1(5):361-378, 1984. 
[57] J. H. Duncan, H. Qiao, V. Philomin, and A. Wenz. Gentle spilling breakers:crest profile evolution. J. Fluid Mech., 379:191-222, 1999.

[58] T.F. Dupont, R.E. Goldstein, L.P. Kadanoff, and S.M. Zhou. Finite-time singularity formation in Hele-Shaw systems. Physical Review E, 47(6):4182-4196, 1993.

[59] N. Ebuchi, H. Kawamure, and Y. Toba. Fine structure of laboratory wind-waves surfaces studied using an optical method. Boundary Layer Met., 39:133-151, 1987.

[60] P. Fast. Dynamics of interfaces in non-Newtonian Hele-Shaw flow. PhD thesis, New York University, 1999.

[61] P. Fast, L. Kondic, M. Shelley, and P. Palffy-Muhoray. Pattern formation in non-Newtonian Hele-Shaw flow. submitted, Physics of Fluids, 1999.

[62] P. Fast and M. Shelley. An overset grid method for free surface flow. LLNL Report, UCRL$J C-136484,1999$.

[63] K.A. Gillow and S.D Howison. A bibliography of free and moving boundary problems for Hele-Shaw and Stokes flow. http://www.maths.ox.ac.uk/ howison/Hele-Shaw/.

[64] M. Glozman, Y. Agnon, and M. Stiassnie. High-order formulation of the water wave problem. Phys. Rev. D, 66:347, 1993.

[65] R. Goldstein and S. Langer. Nonlinear dynamics of stiff polymers. Phys. Rev. Lett., 75:1094, 1995.

[66] R. Goldstein, A. Pesci, and M. Shelley. Topology transitions and singularities in viscous flows. Phys. Rev. Lett., 70:3043, 1993.

[67] R.E. Goldstein, A.I. Pesci, and M.J. Shelley. Topology transitions and singularities in viscous flows. Physical Review Letters, 70(20):3043-3046, 1993.

[68] R.E. Goldstein, A.I. Pesci, and M.J. Shelley. Attracting manifold for a viscous topology transition. Physical Review Letters, 75(20):3665-3668, 1995.

[69] R.E. Goldstein, A.I. Pesci, and M.J. Shelley. Instabilities and singularities in Hele-Shaw flow. Physics of Fluids, 10(11):2701-2723, 1998.

[70] R.E. Goldstein, A.I. Pesci, and M.J. Shelley. Domain of convergence of perturbative solutions for Hele-Shaw flow near interface collapse. Physics of Fluids, Letters, 11(10):2809-2811, 1999.

[71] R.E. Goldstein, T.R. Powers, and C.H. Wiggins. Viscous nonlinear dynamics of twist and writhe. Phys. Rev. Lett., 80:5232-5235, 1998.

[72] A. Greenbaum, L. Greengard, and G.B. McFadden. Laplace's equation and the Dirichlet-Neumann map in multiply connected domains. J. Comp. Phys., 105:267, 1993.

[73] L. Greengard and J. Helsing. On the numerical evaluation of elastostatic fields in locally isotropic two-dimensional composites. J. Mech. Phys. Solids, 46:1441-1462, 1998.

[74] L. Greengard and V. Rokhlin. A fast algorithm for particle summations. J. Comp. Phys., 73:325, 1987. 
[75] H. Hadavinia, S.G. Advani, and R.T. Fenner. The evolution of radial fingering in a Hele-Shaw cell using C-1 continuous overhauser boundary-element method. Engineering Analysis with Boundary Elements, 16(2):183-195, 1995.

[76] D. Haroldsen and D. Meiron. Numerical calculation of three-dimensional interfacial potential flows using the point vortex method. SIAM J. Sci. Comp., 20:648-683, 1998.

[77] H.S. Hele-Shaw. The flow of water. Nature, 58(1489):33-36, 1898.

[78] C. Herring. Surface tension as a motivation for sintering. In W.E. Kingston, editor, The physics of powder metallurgy, page 143. McGraw-Hill, New York, 1951.

[79] Y.E. Hohlov and S.D. Howison. On the classification of solutions to the zero-surface-tension model for Hele-Shaw free-boundary flows. Quarterly of Applied Mathematics, 51(4):777-789, 1993.

[80] T. Hou, I. Klapper, and H. Si. Removing the stiffness of curvature in computing 3-d filaments. Journal of Computational Physics, 143:628-664, 1998.

[81] T. Hou, J. Lowengrub, and M. Shelley. Removing the stiffness from interfacial flows with surface tension. J. Comp. Phys., 114:312-338, 1994.

[82] T. Hou, J. Lowengrub, and M. Shelley. The long-time motion of vortex sheets with surface tension. Phys. Fluids, 9:1933-1954, 1997.

[83] T. Y. Hou and P. Zhang. Stability of a boundary integral method for 3-D water waves. preprint, submitted to SIAM J. Numer. Anal., 1998.

[84] T. Y. Hou and P. Zhang. A new stabilizing technique for boundary integral methods. to appear, Math. Comput., 2000.

[85] T.Y. Hou. Numerical solutions to free boundary problems. Acta Numerica, page 335, 1995.

[86] T.Y. Hou, Z.L. Li, S. Osher, and H.K. Zhao. A hybrid method for moving interface problems with application to the Hele-Shaw flow. Journal of Computational Physics, 134(2):236-252, 1997.

[87] S.D. Howison, A.A. Lacey, and J.R. Ockendon. Hele-Shaw free-boundary problems with suction. Q. JI Mech. Appl. Math., 41:183-193, 1988.

[88] J.M. Hyman. Numerical methods for tracking fluid interfaces. Physica D, 12:396, 1984.

[89] J. Ignesmullol and J.V. Maher. Experiments on anisotropic radial viscous fingering. Physical Review E, 53(4B):3788-3793, 1996.

[90] T. Imaeda and K. Kawasaki. Multipole expansion in the ostwald ripening theory. Physica A, 164:335-352, 1990.

[91] W.C. Johnson and J.I.D. Alexander. Interfacial conditions for thermomechanical equilibrium in two-phase crystal. J. Appl. Phys., 59:2735, 1986.

[92] W.C. Johnson and P.W. Voorhees. Elastically-induced precipitate shape transitions in coherent solids. Solid State Phenom., 23:87, 1992. 
[93] H.-J. Jou, P.H. Leo, and J.S. Lowengrub. Microstructural evolution in inhomogeneous elastic media. J. Comp. Phys., 131:109-148, 1997.

[94] M.J. Kaufman, P.W. Voorhees, W.C. Johnson, and F.S. Biancaniello. An elastically induced morphological instability of a misfitting precipitate. Metall. Trans. A, 20A:2171-2175, 1989.

[95] J.B. Keller and M. Miksis. Surface tension drive flows. SIAM J. Appl. Math., 43:268, 1983.

[96] O.D. Kellogg. Foundations of Potential Theory. J. Springer, Berlin, 1929.

[97] E.D. Kelly and E.J. Hinch. Numerical simulations of sink flow in the Hele-Shaw cell with small surface tension. Euro. Jnl. of Applied Maths, 8(6):533-550, 1997.

[98] E.D. Kelly and E.J. Hinch. Numerical solution of Hele-Shaw flows driven by a quadrupole. European Journal of Applied Mathematics, 8(6):551-566, 1997.

[99] A.G. Khachaturyan, S.V. Semenovskaya, and J.W. Morris Jr. Theoretical analysis of straininduced shape changes in cubic precipitates during coarsening. Acta Metall., 36:1563, 1988.

[100] C.V. Kietzmann, J.P. Vanderwalt, and Y.S. Morsi. A free-front tracking algorithm for a control-volume-based Hele-Shaw method. International Journal for Numerical Methods in Engineering, 41(2):253-269, 1998.

[101] L. Kondic, P. Palffy-Muhoray, and M.J. Shelley. Models of non-Newtonian Hele-Shaw flow. Physical Review E, 54(5):R4536-R4539, 1996.

[102] L. Kondic, M.J. Shelley, and P. Palffy-Muhoray. Non-Newtonian Hele-Shaw flow and the Saffman-Taylor instability. Physical Review Letters, 80(7):1433-1436, 1998.

[103] R. Krasny. Desingularization of vortex sheet roll-up. J. Comp. Phys., 65:292, 1986.

[104] R. Krasny. A study of singularity formation in a vortex sheet by the point vortex approximation. J. Fluid Mech., 167:65, 1986.

[105] H. Kudela. The influence of surface tension effects on using vortex method in the study of Rayleigh-Taylor instability. In Notes on Numerical Fluid Mechanics. Vieweg-Verlag, Braunschweig, Wiesbaden (Germany), 1990.

[106] F.C. Larchè and J.W. Cahn. A linear theory of thermochemical equilibrium of solids under stress. Acta Metall., 21:1051, 1973.

[107] J. Lee. Elastic stress and microstructural evolution. Mat. Trans. Jim, 39:114, 1998.

[108] Jong K. Lee. A study on coherency strain and precipitate morphology via a discrete atom method. to appear Metall. Trans. A.

[109] Jong K. Lee. Coherency strain analysis via a dicrete atom method. Scripta Met., 32:559, 1995.

[110] P. Leo, J. Lowengrub, and H.-J. Jou. A diffuse interface model for microstructural evolution in elastically stressed solids. Aca Metall., 46:2113-2130, 1998.

[111] P. Leo, J. Lowengrub, and Q. Nie. On an elastically induced splitting instability. in preparation. 
[112] P. Leo, J. Lowengrub, and Q. Nie. Microstructural evolution in inhomogeneous elastic media. J. Comp. Phys., 157:44-88, 2000.

[113] P.H. Leo and R.F. Sekerka. The effect of surface stress on crystal-melt and crystal-crystal equilibrium. Acta Metall., 37:3119, 1989.

[114] D. Li and Q. Chen. Selective variant growth of coherent $t i_{11} n i_{14}$ precipitates in a tini alloy under applied stress. Aca Metall., 45:471, 1997.

[115] D. Li and Q. Chen. Shape evolution and splitting of coherent particles under applied stress. Aca Metall., 47:247, 1998.

[116] S. Liang. Random walk simulations of flow in Hele Shaw cells. Physical Review A, 33(4):2663$2674,1986$.

[117] I.M. Lifshitz and V.V. Slyozov. J. Phys. Chem. Solids, 19:35, 1961.

[118] M. S. Longuet-Higgins. Parasitic capillary waves: a direct calculation. J. Fluid Mech., 301:79-107, 1995.

[119] M. S. Longuet-Higgins. Capillary jumps on deep water. Journal of Physical Oceanography, 26:1957-1965, 1996.

[120] M. S. Longuet-Higgins and E. D. Cokelet. The deformation of steep surface waves on water I. A numerical method of computation. Proc. R. Soc. Lond. A., 350:1-26, 1976.

[121] T.S. Lundgren and N.N. Mansour. Oscillations of drops in zero gravity with weak viscous effects. J. Fluid Mech., 194:479, 1988.

[122] A. Maheshwari and A.J. Ardell. Morphological evolution of coherent misfitting precipitates in anisotropic elastic media. Phys. Rev. Lett., 70:2305, 1993.

[123] N.N. Mansour and T.S. Lundgren. Satellite formation in capillary jet breakup. Phys. Fluids A, 2:1141, 1990 .

[124] K.V. McCloud and J.V. Maher. Experimental perturbations to Saffman-Taylor flow. Physics Reports, 260(3):139-185, 1995.

[125] M. McCormack, A.G. Khatchaturyan, and J.W. Morris. A two-dimensional analysis of the evolution of coherent precipitates in elastic media. Acta Metall., 40:325, 1992.

[126] G.B. McFadden, P.W. Voorhees, R.F. Boisvert, and D.I. Meiron. A boundary integral method for the simulation of two-dimensional particle coarsening. J. Sci. Comp., 1:117, 1986.

[127] E. Meiburg and G.M. Homsy. Nonlinear unstable viscous fingers in Hele-Shaw flows. 2: Numerical simulation. Physics of Fluids, 31(3):429-439, 1988.

[128] D. Meiron, G. Baker, and S. Orszag. Analytic structure of vortex sheet dynamics. part 1. Kelvin-Helmholtz instability. J. Fluid Mech., 114:283, 1982.

[129] S.G. Mikhlin. Integral Equations and Their Applications to Certain Problems in Mechanics, Mathematical Physics, and Technology. Pergamon Press, New York, 1957. 
[130] T. Miyazaki, K. Hakamura, and H. Mori. Experimental and theoretical investigations on morphological changes of gamma precipitates in Ni-Al single crystals during uniaxial stressannealing. J. Mater. Sci., 14:1827, 1979.

[131] D. Moore. The spontaneous appearance of a singularity in the shape of an evolving vortex sheet. Proc. R. Soc. Lond. A, 365:1059, 1979.

[132] D.W. Moore. On the point vortex method. SIAM J. Sci. Stat. Comput., 2:65, 1981.

[133] R. Mueller and D. Gross. 3d simulation of equilibrium morphologies of precipitates. Comp. Materials Sci., 11:35, 1998.

[134] A.L. New, P. McIver, and D.H. Peregrine. Computations of overturning waves. J. Fluid Mech., 150:233, 1985.

[135] Q. Nie. The nonlinear evolution of vortex sheets with surface tension in axisymmetric flows. in preparation.

[136] Q. Nie and G. Baker. Application of adaptive quadrature to axi-symmetric vortex sheet motion. J. Comput. Phys., 143:49-69, 1998.

[137] Q. Nie and F. R. Tian. Singularities in Hele-Shaw flows. SIAM J. Appl. Math, 58(1):34-54, 1998.

[138] M. Nitsche. Axisymmetric vortex sheet motion: Accurate evaluation of the principal value integral. to appear in SIAM J. Sci. Comput., 1999.

[139] H. Oguz and A. Prosperetti. Bubble entrainment by the impact of drops on liquid surfaces. J. Fluid Mech., 219:143, 1990.

[140] D. Orlikowski, C. Sagui, A. Somoza, and C. Roland. Large-scale simulations of phase separation of elastically coherent binary systems. Phys. Rev. E, 59:983, 1999.

[141] W. Ostwald. Z. Phys. Chem., 34:495, 1900.

[142] M. Perlin, H. Lin, and C.-L. Ting. On parasitic capillary waves generated by steep gravity waves: an experimental investigation with spatial and temporal measurements. J. Fluid Mech., 255:597-620, 1993.

[143] E. Poire and M. Ben Amar. Finger behavior of a shear thinning fluid in a Hele-Shaw cell. Physical Review Letters, 81:2048-2051, 1998.

[144] H. Power. The evolution of radial fingers at the interface between 2 viscous liquids. Engineering Analysis with Boundary Elements, 14(4):297-304, 1994.

[145] A. Prosperetti and H. Oguz. The impact of drops on liquid surfaces and the underwater noise of rain. Annu. Rev. Fluid Mech., 25:577-602, 1993.

[146] N. Provatas, N. Goldenfeld, J. Dantzig, J. LaCombe, A. Lupulescu, M. Koss, M. Glicksman, and R. Almgren. Crossover scaling in dendritic evolution a low undercooling. Physical Review Letters, 82(22):4496-4499, 1999.

[147] D.A. Pugh. Development of vortex sheets in boussinesq flows. Ph.D. thesis, Department of Mathematics, Imperial College, London, 1989. 
[148] M.C. Pugh and M.J. Shelley. Singularity formation in thin jets with surface tension. CPAM, 51:733, 1998.

[149] D.I. Pullin. Numerical studies of surface tension effects in nonlinear Kelvin-Helmholtz and Rayleigh-Taylor instability. J. Fluid Mech., 119:507, 1982.

[150] G. Purdy. The theory of diffusional phase transformations and microstructure. Metallography, $7: 131,1975$.

[151] R.H. Rangel and W.A. Sirignano. Nonlinear growth of Kelvin-Helmholtz instability: Effect of surface tension and density ratio. Phys. Fluids, 31(7):1845-1855, 1988.

[152] M. Reed and B. Simon. Functional Analysis, volume 1 of Methods of Modern Mathematical Physics. Academic Press, New York, 1980.

[153] F.J. Rizzo and D.J. Shippy. A formulation and solution procedure for the general nonhomogeneous elastic inclusion problem. Int. J. Solids Structures, 4:1161-1179, 1968.

[154] F.J. Rizzo and D.J. Shippy. A method for stress determination in plane anisotropic bodies. J. Comp. Mater., 4:36, 1970.

[155] A. J. Roberts. A stable and accurate numerical method to calculate the motion of a sharp interface between fluids. IMA J. Appl. Math., 31:13-35, 1983.

[156] P.B. Robinson and J.M. Boulton-Stone. Application of the boundary integral method to the interaction of rising two dimensional deformable gas bubbles. J. Engrg. Math, 29:393, 1995.

[157] R. Rogallo. NASA TM-73203 (unpublished).

[158] J. E. Romate. The numerical simulation of nonlinear gravity waves. Engrng. Analysis Bdry. Elts., 7:156-166, 1990.

[159] J. E. Romate and P. J. Zanbergen. Boundary integral equation formulations for free-surface flow problems in two and three dimensions. Comput. Mech., 4:267-282, 1989.

[160] L. Rosenhead. The point vortex approximation of a vortex sheet. Proc. Roy. Soc. London Ser. A, 134:170-192, 1932.

[161] J.W. Rottman and D.B. Olfe. Comment on discretized simulations of vortex sheet evolution with buoyancy and surface tension effects. AIAA J., 15:1214, 1977.

[162] G. Rubin and A. Khachaturyan. Three dimensional model of precipitation of ordered metallics. Acta Mater., 47:1995, 1999.

[163] Y. Saad and M.R. Schultz. GMRES: A generalized minimum residual method for solving nonsymmetric linear systems. SIAM J. Sci. Stat. Comput., 7:856, 1986.

[164] P. Saffman and G. Baker. Vortex interactions. Annu. Rev. Fluid Mech., 11:95, 1979.

[165] P. G. Saffman and G. I. Taylor. The penetration of fluid into a porous medium or Hele-Shaw cell. Proc. Roy. Soc. A., 245:312-329, 1958.

[166] T. Sarpkaya. Vorticity, free surface, and surfactants. Annu. Rev. Fluid Mech., 28:83-128, 1996. 
[167] R. Scardovelli and S. Zaleski. Direct numerical simulation of free-surface and interfacial flow. Annu. Rev. Fluid Mech., 31:567-603, 1999.

[168] I. Schmidt and D. Gross. The equilibrium shape of an elastically inhomogeneous inclusion. J. Mech. Phys. Solids, 45:1521, 1997.

[169] I. Schmidt, R. Mueller, and D. Gross. The effect of elastic inhomogeneity on equilibrium and stability of a two particle morphology. Mech. Mater., 30:181, 1998.

[170] L. W. Schwartz and J. D. Fenton. Strongly nonlinear waves. Ann. Rev. Fluid Mech., 14:39-60, 1983.

[171] D. Schwendeman. A new numerical method for shock wave propagation based on geometrical shock dynamics. Proceedings of the Royal Society of London, A441:331-341, 1993.

[172] M.J. Shelley. A study of singularity formation in vortex sheet motion by a spectrally accurate vortex method. J. Fluid Mech., 244:493, 1992.

[173] M.J. Shelley, R.E. Goldstein, and A.I. Pesci. Topological transitions in Hele-Shaw flow. In R.E. Caflisch and G.C. Papanicolaou, editors, Singularities in Fluids, Plasmas and Optics, pages 167-188. Kluwer, 1993.

[174] M.J. Shelley, F. Tian, and K. Wlodarski. Hele-Shaw flow and pattern formation in a timedependent gap. Nonlinearity, 10(6):1471-1495, 1997.

[175] M.J. Shelley and T. Ueda. The nonlocal dynamics of stretching, buckling filaments. In D.T. Papageorgiou Y.Y. Renardy, A.V. Coward and S-M. Sun, editors, Advances in MultiFluid Flows, pages 415-425, Philadelphia, 1996. SIAM.

[176] M.J. Shelley and T. Ueda. The stokesian hydrodynamics of flexing, stretching filaments. Physica D, to appear, 2000.

[177] D.I. Sherman. On the problem of plain strain in non-homogeneous media. In W. Olszak, editor, Non-homogeneity in Elasticity and Plasticity, pages 3-20. Pergamon Press, London, 1959 .

[178] A. Sidi and M. Israeli. Quadrature methods for periodic singular and weakly singular fredholm integral equations. J. Sci. Comp., 3:201-231, 1988.

[179] M. Siegel, S. Tanveer, and W.-S. Dai. Singular effects of surface tension in evolving Hele-Shaw flows. J. Fluid Mech., 323:201-236, 1996.

[180] H. Stone. Dynamics of drop deformation and breakup in viscous fluids. Annu. Rev. Fluid Mech., 26:65-102, 1994.

[181] J. Strain. A boundary integral approach to unstable solidification. J. Comp. Phys., 85:342, 1989.

[182] C.H. Su and P. Voorhees. The dynamics of precipitate evolution in elastically stressed solids: I. inverse coarsening. Acta Mater., 44:1987, 1996.

[183] C.H. Su and P. Voorhees. The dynamics of precipitate evolution in elastically stressed solids: II. particle alignment. Acta Mater., 44:2000, 1996. 
[184] S. Tanveer. Evolution of Hele-Shaw interface for small surface tension. Phil. Trans. R. Soc. Lond. A, 343:155-204, 1993.

[185] S. Tanveer. Surprises in viscous fingering. J. of Fluid Mech., 409:273-308, 2000.

[186] M. Thompson and P. Voorhees. Spinodal decomposition in elastically anistropic inhomogeneous systems in the presence of applied traction. Modell. Sim. Mat. Sci. Eng., 5:223, 1997.

[187] M. Thompson and P. Voorhees. Equilibrium particle morphologies in elastically stressed coherent solids. Acta Mater., 47:983, 1999.

[188] M.E. Thompson, C.S. Su, and P.W. Voorhees. The equilibrium shape of a misfitting precipitate. Acta Metall., 42:2107, 1994.

[189] K. Thornton, N. Akaiwa, and P. Voorhees. to appear in Proceedings of the international conference on solid-solid phase transformations, Kyoto, Japan, 1999.

[190] K. Thornton, N. Akaiwa, and P. Voorhees. Morphology development during ostwald ripening in elastically stressed solids. In Y-W. Chung, D.C. Dunand, P.K. Liaw, and G.B. Olson, editors, Advanced Materials for the 21st Century, pages 205-212. The Minerals, Metals and Materials Society, Pennsylvania, 1999.

[191] K. Thornton, N. Akaiwa, and P. Voorhees. in preparation, 2000.

[192] F.R. Tian. On the breakdown of Hele-Shaw solutions with non-zero surface tension. $J$. Nonlinear Sci., 5:479-484, 1995.

[193] J.K. Tien and S.M. Copley. The effect of uniaxial stress on the periodic morphology of coherent gamma prime precipitates in nickel-base superalloy crystals. Met. Trans., 2:215, 1971.

[194] G. Tryggvason and H. Aref. Numerical experiments in Hele-Shaw flows with a sharp interface. J. Fluid Mech., 136:1-30, 1983.

[195] G. Tryggvason and H. Aref. Finger-interaction mechanisms in stratified Hele-Shaw flow. Journal of Fluid Mechanics, 154:287-301, 1985.

[196] G. Tryggvason and S.O. Unverdi. The shear breakup of an immiscible fluid interface. In W. Shyy and R. Narayanan, editors, Fluid Dynamics at Interfaces, page 142. Cambridge University Press, Cambridge, 1999.

[197] G. Trygvason. Numerical simulations of the Rayleigh-Taylor instability. J. Comp. Phys., $75: 253,1988$.

[198] W.-T. Tsai and D. Yue. Computations of nonlinear free-surface flows. Annu. Rev. Fluid Mech., 28:249-278, 1996.

[199] M. P. Tulin. Breaking of ocean waves and downshifting. In Waves and Nonlinear Processes in Hydrodynamics, pages 177-190. Kluwer Academic Publishers, 1996.

[200] T. Vinje and P. Brevig. Numerical simulation of breaking waves. Adv. Water Resources, 4:77-82, 1981. 
[201] P.W. Voorhees. The theory of Ostwald ripening. J. Stat. Phys., 38:231, 1985.

[202] P.W. Voorhees. Ostwald ripening of two phase mixtures. Ann. Rev. Mater. Sci., 22:197, 1992.

[203] P.W. Voorhees, R.F. Boisvert G.B. McFadden, and D.I. Meiron. Numerical simulation of morphological development during Ostwald ripening. Acta Metall., 36:207, 1988.

[204] P.W. Voorhees, G.B. McFadden, and W.C. Johnson. On the morphological development of second-phase particles in elastically-stressed solids. Acta Metall., 40:2979, 1992.

[205] C. Wagner. Z. Elektrochem., 65:581, 1961.

[206] Y. Wang, L. Chen, and A.G. Khachaturyan. Computer simulation of microstructure evolution in coherent solids. In W.C. Johnson, J.M. Howe, D.E. Laughlin, and W.A. Soffa, editors, Solid-solid phase transformations, page 245. The Minerals, Metals and Materials Society, Pennsylvania, 1994.

[207] Y. Wang, L. Chen H. Wang, and A.G. Khachaturyan. Shape evolution of a coherent tetragonal precipitate in partially stabilized cubic $z \mathrm{r}_{2}$ : a computer simulation. J. Am. Ceram. Soc., 76:3029, 1993.

[208] Y. Wang and A.G. Khachaturyan. Shape instability during precipitate growth in coherent solids. Acta Metall., 43:1837, 1995.

[209] B.J. West, K.A. Bruekner, and R.S. Janda. A new numerical method for surface hydrodynamics. J. Geophys. Res. C, 92:11803, 1987.

[210] N. Whitaker. Numerical solution of the Hele-Shaw equations. Journal of Computational Physics, 90(1):176-199, 1990.

[211] N. Whitaker. Some numerical methods for the Hele-Shaw equations. Journal of Computational Physics, 111(1):81-88, 1994.

[212] G.B. Whitham. A new approach to problems of shock dynamics, part i: Two-dimensional problems. J. Fluid Mech., 2:145, 1957.

[213] S. J. Wu. Well-posedness in Sobolev spaces of the full water wave problem in 2-D. Invent. Math., 130:39-72, 1997.

[214] Y. Yang. The initial value problem of a rising bubble in a two dimensional vertical channel. Phys. Fluids A, 4:913, 1992.

[215] R. W. Yeung. Numerical methods in free-surface flows. Ann. Rev. Fluid Mech., 14:395-442, 1982.

[216] E. Yokoyama and R.F. Sekerka. A numerical study of the combined effect of anisotropic surface tension and interface kinetics on pattern formation during the growth of two dimensional crystals. J. Crystal Growth, 125:389-403, 1992.

[217] Y.S. Yoo, D.Y. Yoon, and M.F. Henry. The effect of elastic misfit strain on the morphological evolution of $\gamma^{\prime}$-precipitates in a model Ni-base superalloy. Metals and Materials, 1:47, 1995.

[218] R. Zalosh. Discretized simulation of vortex sheet evolution with buoyancy and surface tension effects. AIAA J., 14:1517-1523, 1976. 
[219] J. Zhu, X. Chen, and T.Y. Hou. An efficient boundary integral method for the Mullins-Sekerka problem. J. Comp. Phys., 127:246-267, 1996.

[220] J.A. Zufria. Vortex-in-cell simulation of bubble competition in a Rayleigh-Taylor instability. Phys. Fluids, 31:3199, 1988. 


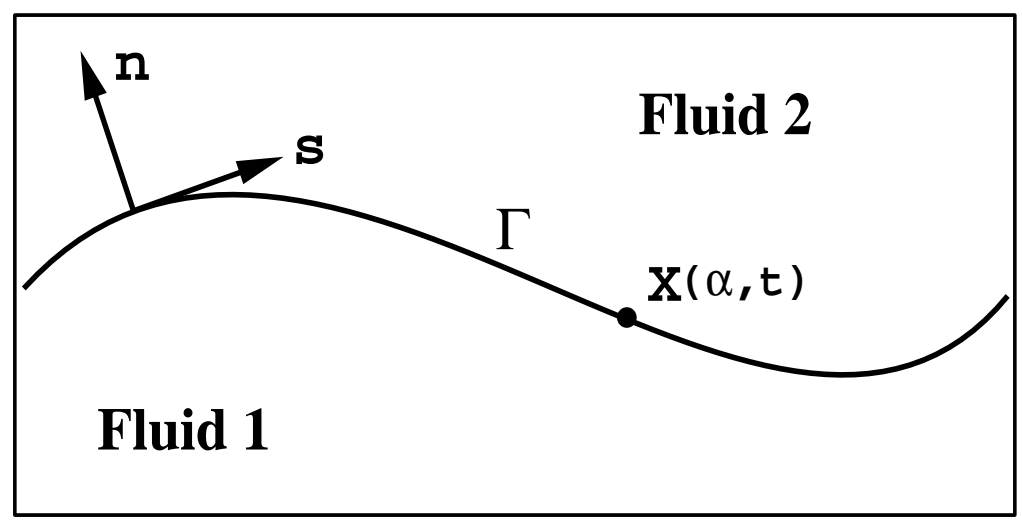

Figure 1: A schematic of an interface $\Gamma$ separating two immiscible fluids.

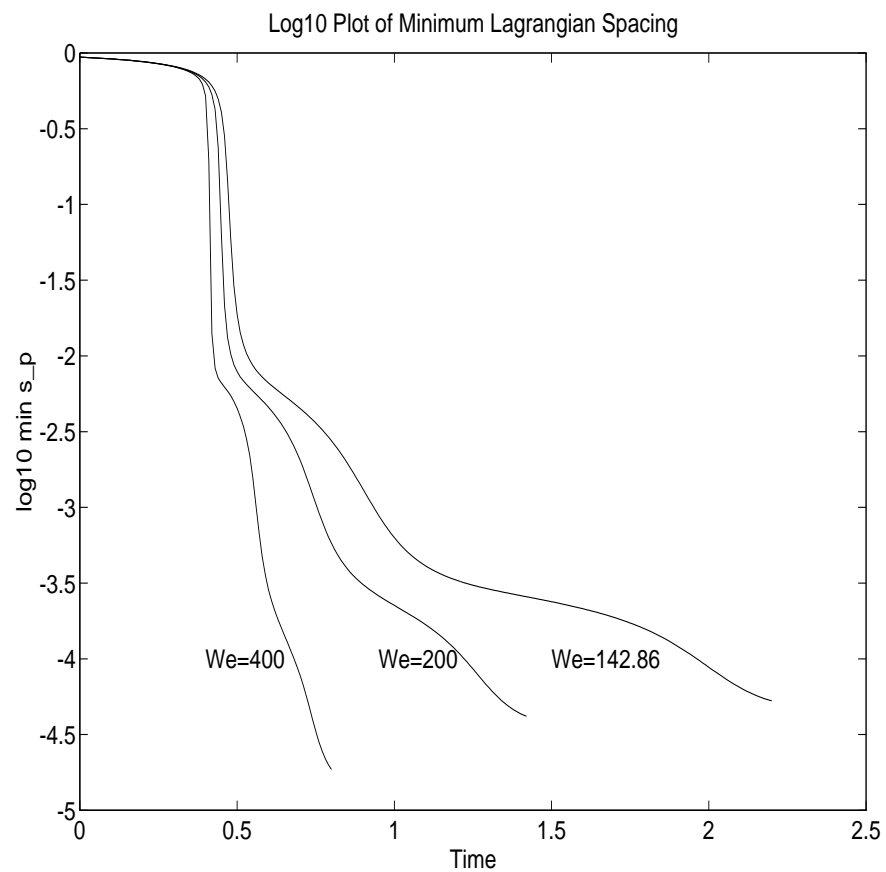

Figure 2: The evolution of $\log _{10}\left(\bar{s}_{\alpha}\right)$ for several Weber numbers. 

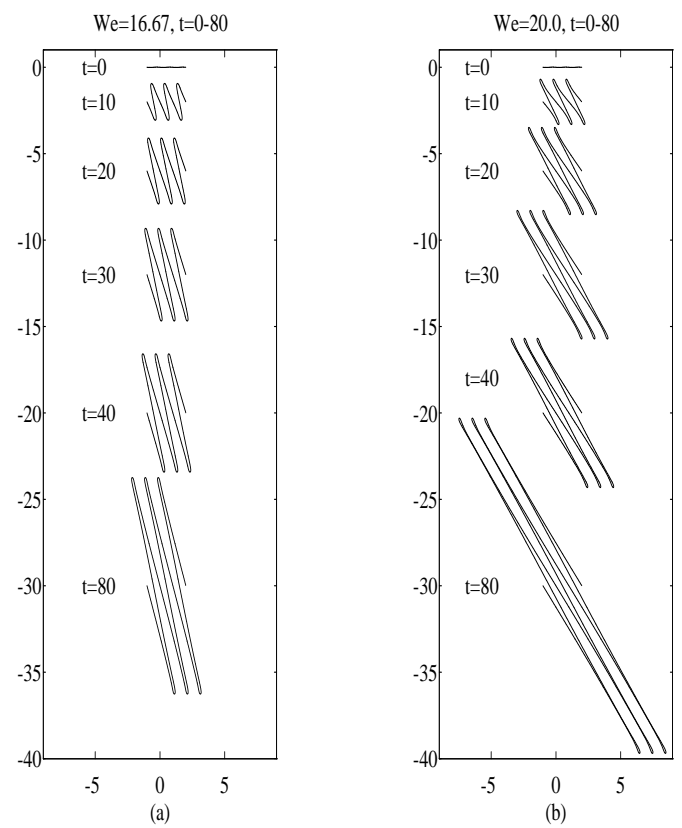

Figure 3: Growing fingers of interpenetrating fluid for $W e=16.67$ and 20. Three spatial periods are shown at each time.
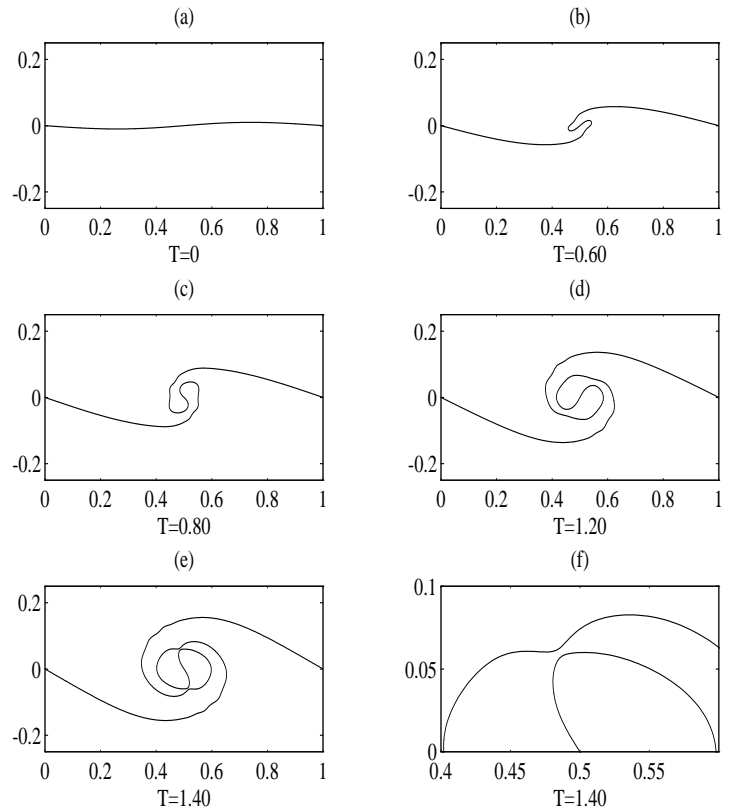

Figure 4: The long-time evolution from a nearly flat sheet for $W e=200$. The bottom right box shows a close-up of the thinning neck at $t=1.4$. 

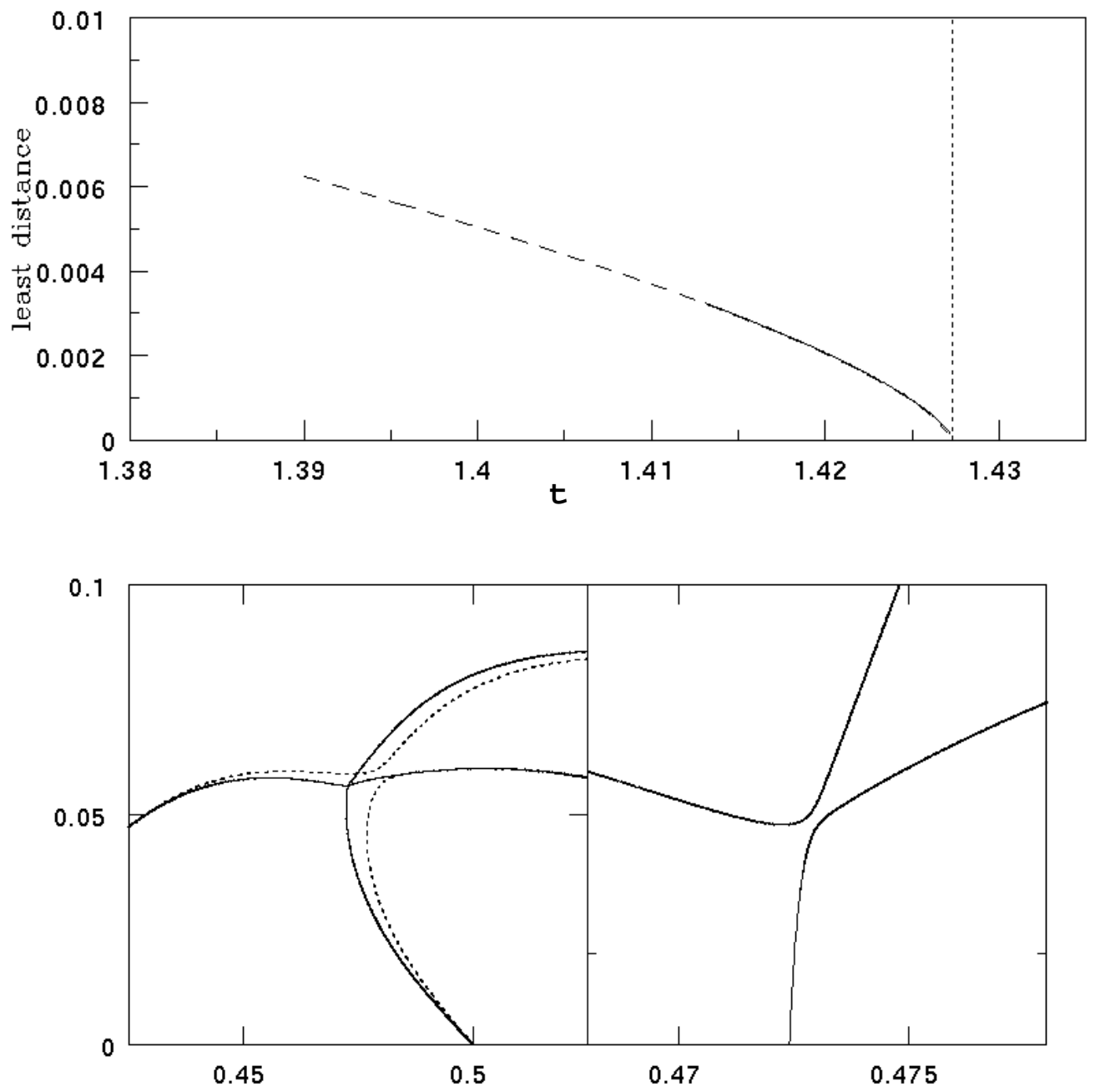

Figure 5: The top figure shows the collapse of the neck width in time. In the bottom figure, the left box shows a close-up of the upper pinching region of the rolled-up sheet at times $t=1.4135$ (dashed) and 1.427 (solid), both very near to the collapse time. The right box magnifies this close-up by another factor of 10 . 

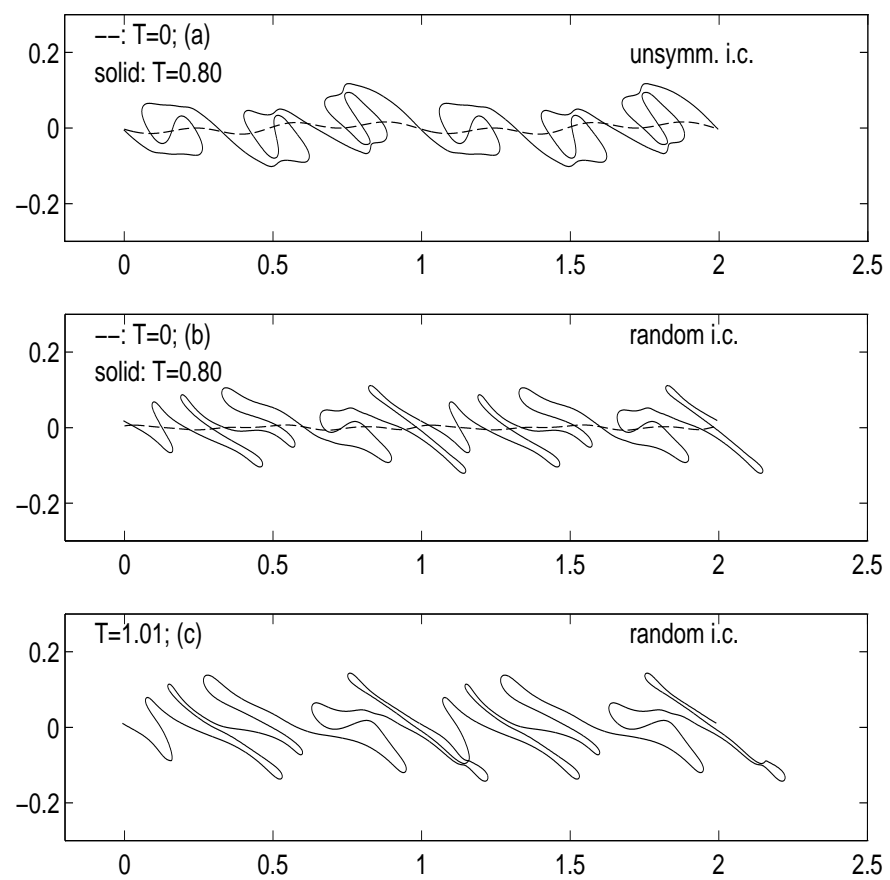

Figure 6: The development of the Kelvin-Helmholtz instability $(W e=200)$ shown over two spatial periods, for various initial data. (a): The initial data is in the $k=1$ and 3 modes, each with a randomly chosen phase. (b) and (c): The initial data is in the first 30 modes, with randomly chosen initial amplitudes and phases.

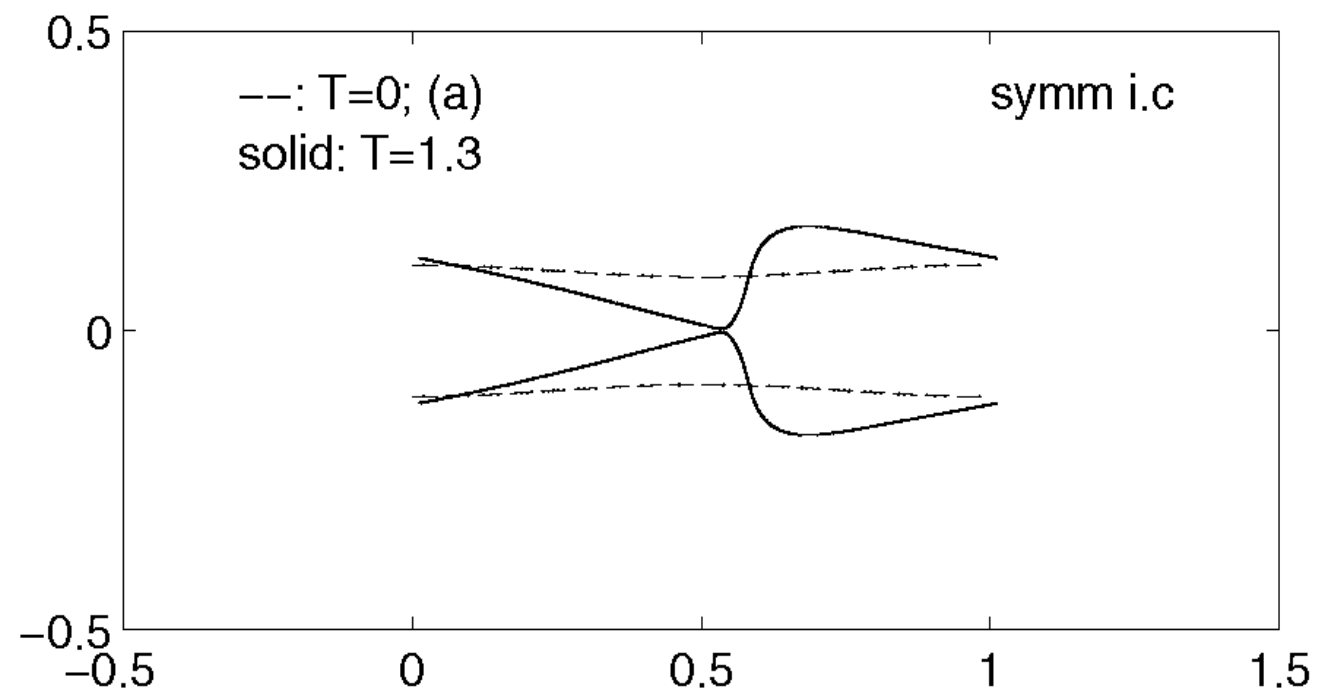

Figure 7: The collapse of a jet between two interfaces under surface tension. The dashed curves show the initial position. 

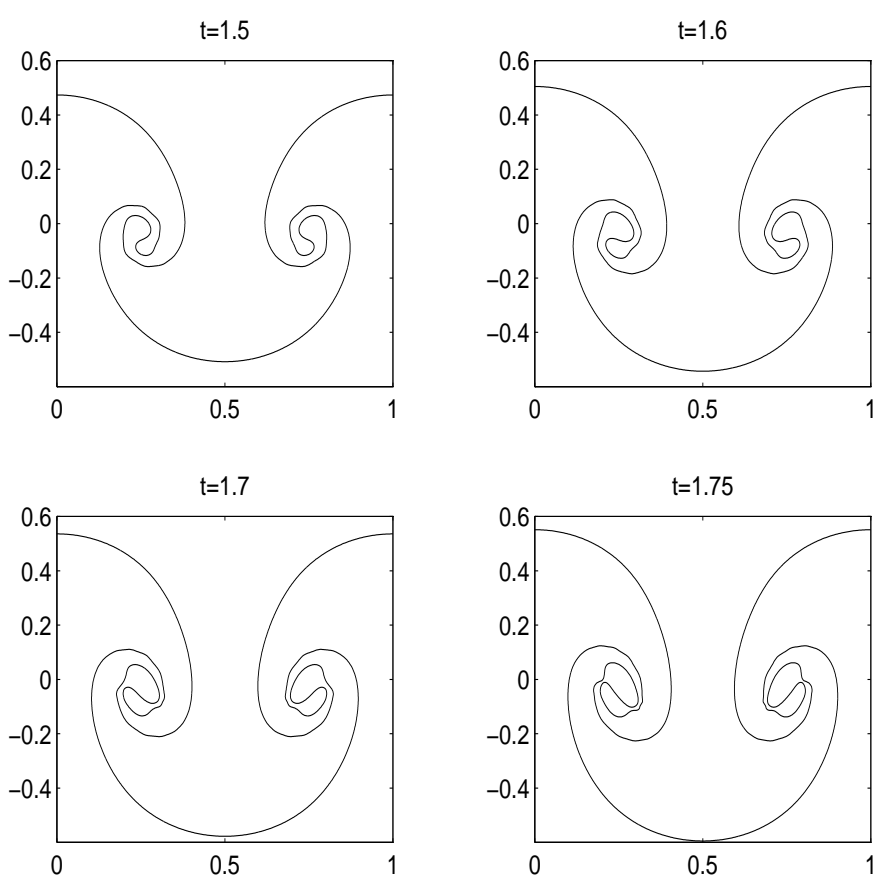

Figure 8: Unstably stratified flow: $A=-0.1$ and $S=0.005$. Sequence of interface positions. $N=2048$ and $\Delta t=1.25 \times 10^{-4}$. Graph courtesy of Ceniceros \& Hou [33].
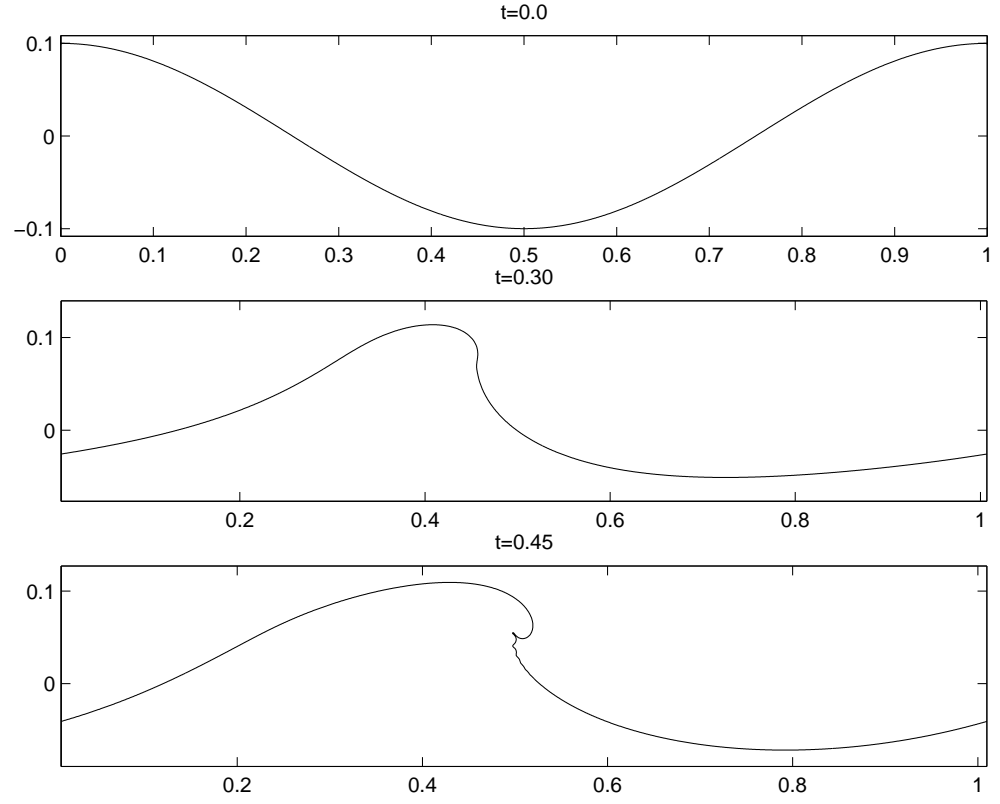

Figure 9: The approach to breaking for a wave with surface tension. Wave profiles shown at times $t=0,0.30$, and 0.45 , using $N=2048$ and $\Delta t=5 \times 10^{-5}$. Graph courtesy of Ceniceros \& Hou [34]. 

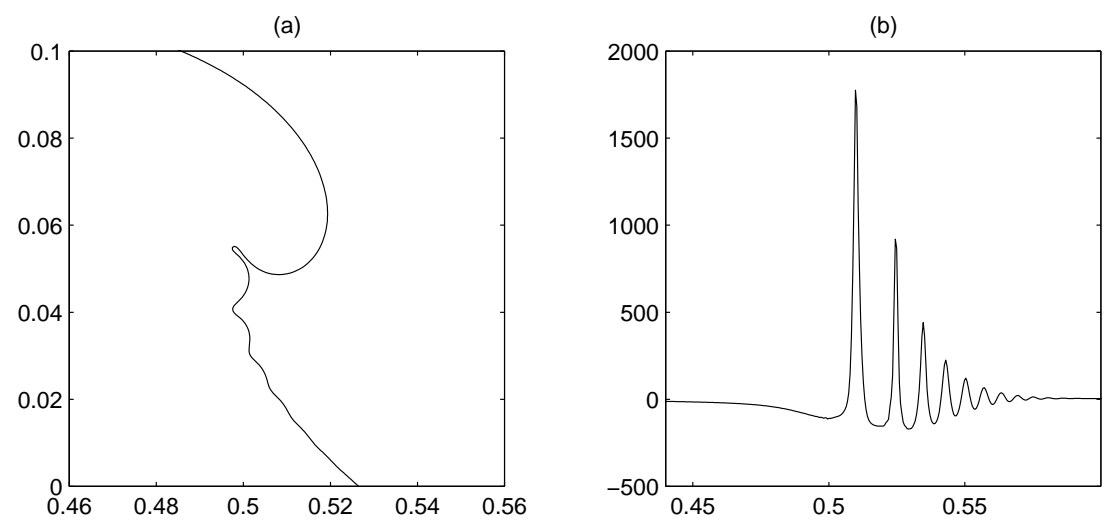

Figure 10: (a) A close-up of the interface at $t=0.45$ and (b) the interfacial curvature plotted against the Lagrangian parameter $\alpha$. Graph courtesy of Ceniceros \& Hou [34].

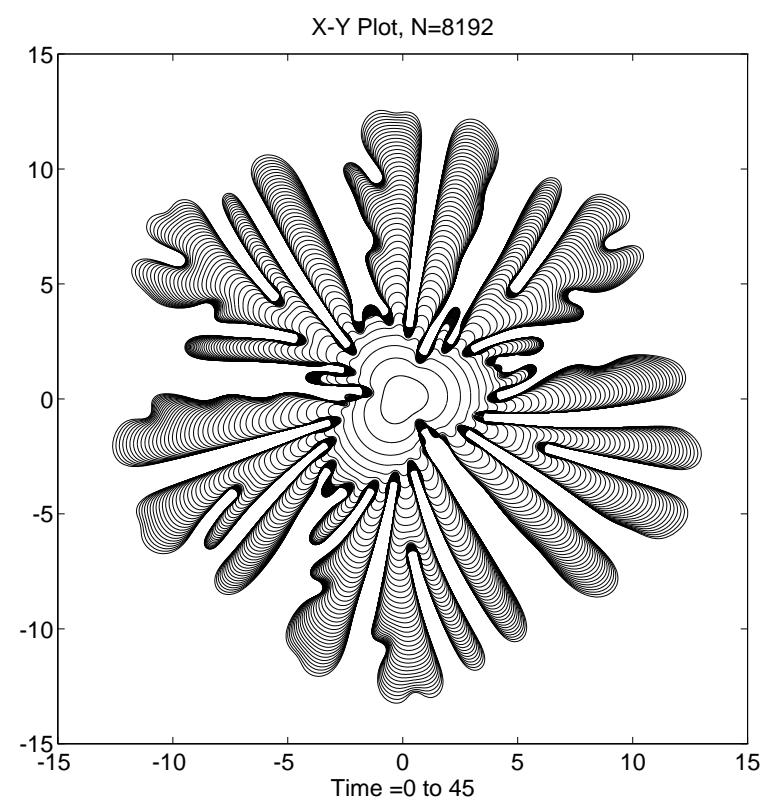

Figure 11: The evolution of an expanding gas bubble in a Hele-Shaw cell. 


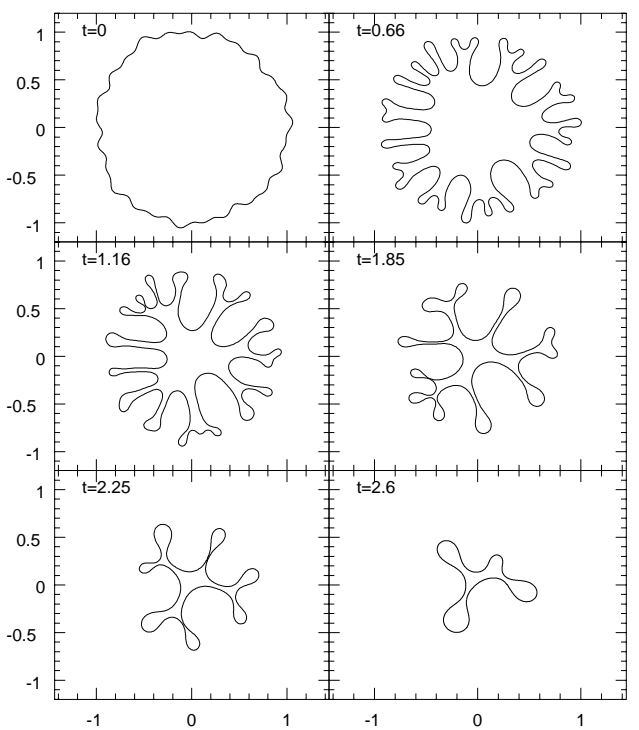

Figure 12: The evolution of a contracting fluid blob as the cell gap-width is increased in time. Graph courtesy of Shelley, Tian, \& Wlodarski [174].
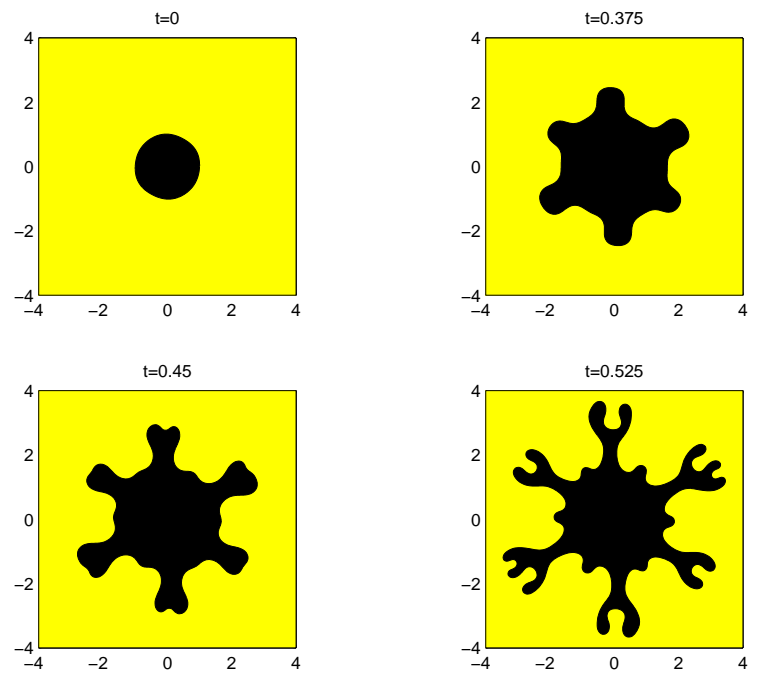

Figure 13: The centrifugal instability of a liquid bubble in a rotating Hele-Shaw cell. Graph courtesy of Lowengrub \& Shelley (unpublished). 


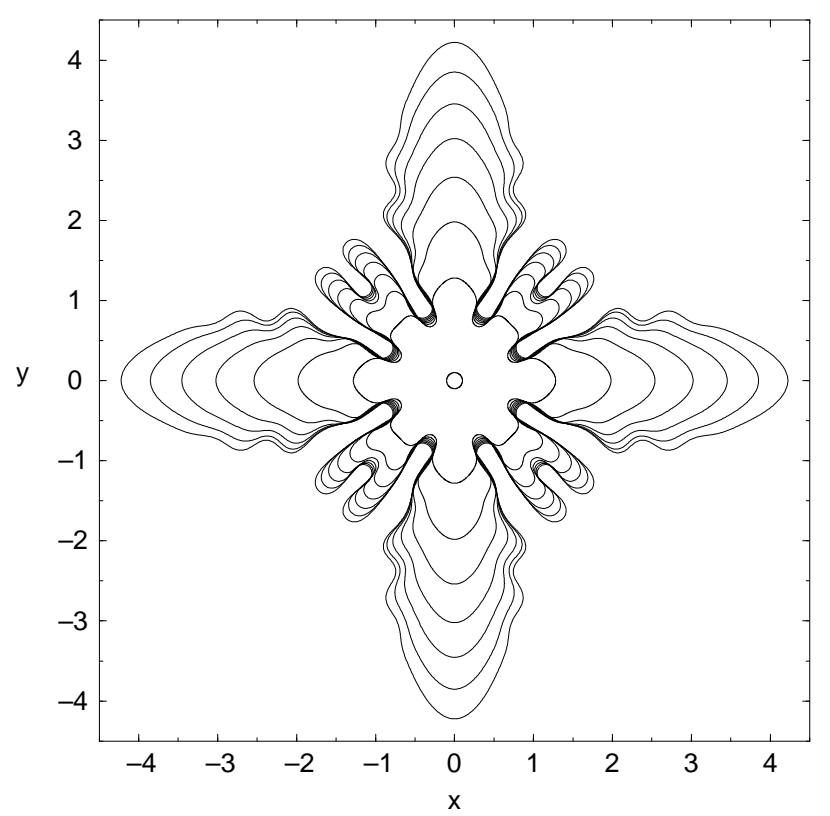

Figure 14: Pattern formation in an anisotropic Hele-Shaw cell. Graph courtesy of Almgren, Dai, \& Hakim [4].
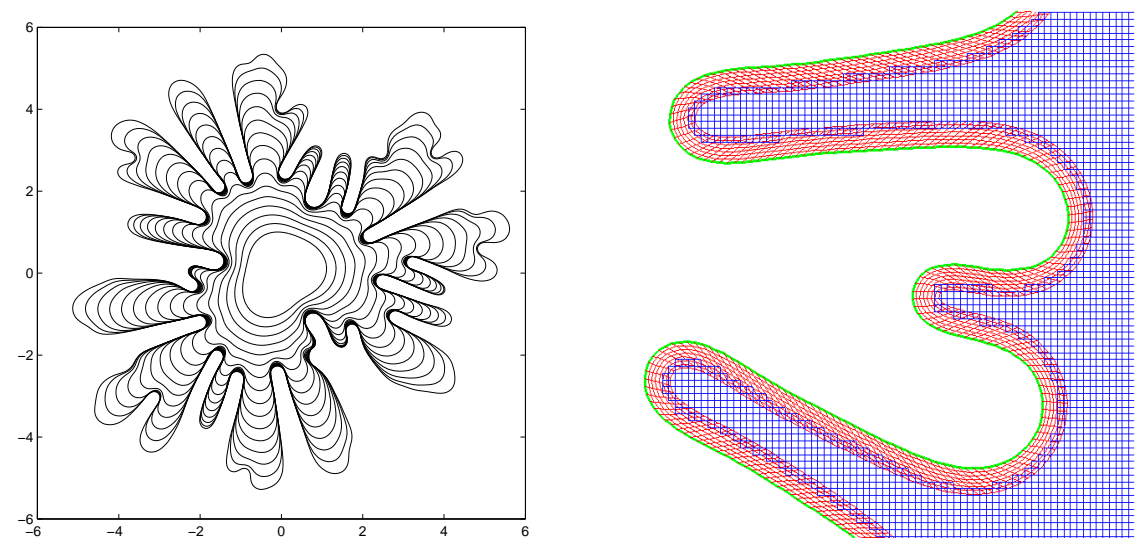

Figure 15: Left box: A simulation of the development of the S-T instability for a slightly shearthinning fluid. Right box: A closeup of the overset grids used in the simulation, where the bodyfitted grid is coupled to the Cartesian background grid. Graph courtesy of Fast \& Shelley [62]. 


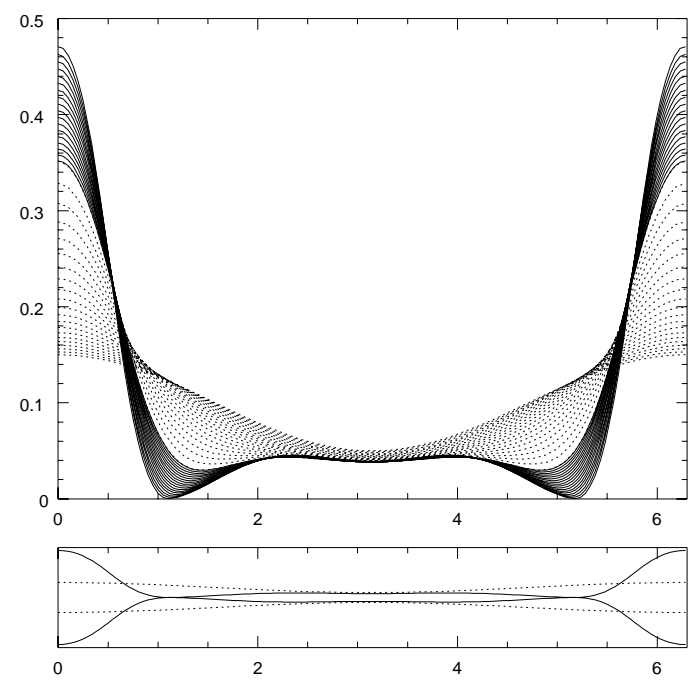

Figure 16: The collapse of a thin fluid layer, under an unstable density stratification, in the HeleShaw cell. The lower graph shows the "final" interface in a common $x-y$ scale. Courtesy of Shelley, Goldstein, \& Pesci [66]. 


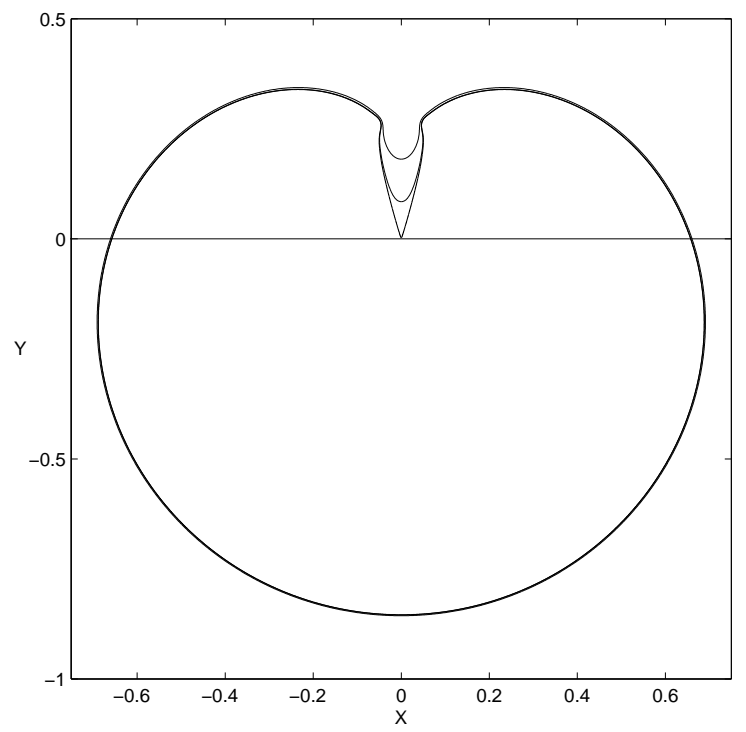

Figure 17: The evolution of the initially circular fluid blob, for $S=5 \times 10^{-5}$, past the "cusp" time $t_{c}=0.2842$. Times shown: $t=0.2880,0.2900$, and 0.29181. $N=16384$ and $\Delta t=2 \times 10^{-7}$ for the last stage of the motion. (cf. Fig. 2 of Nie \& Tian [137]). Graph courtesy of Ceniceros, Hou, \& Si $[36]$.

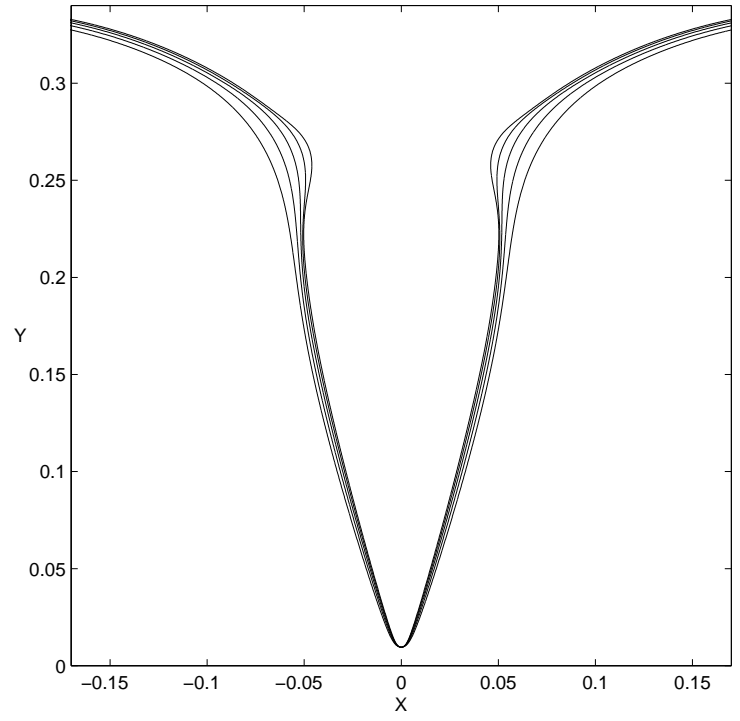

Figure 18: Comparison of the interface finger for a sequence of surface tensions. From the outer curve inwards, the fingers correspond to the surface tension values $S=8 \times 10^{-4}, 4 \times 10^{-4}, 2 \times 10^{-4}$, $1 \times 10^{-4}$, and $5 \times 10^{-5}$. Each interface is plotted when the tip of the finger reaches the fixed level $y=0.01$ at $x=0 . N=16384$ and $\Delta t=2 \times 10^{-7}$. Graph courtesy of Ceniceros, Hou, \& Si [36]. 

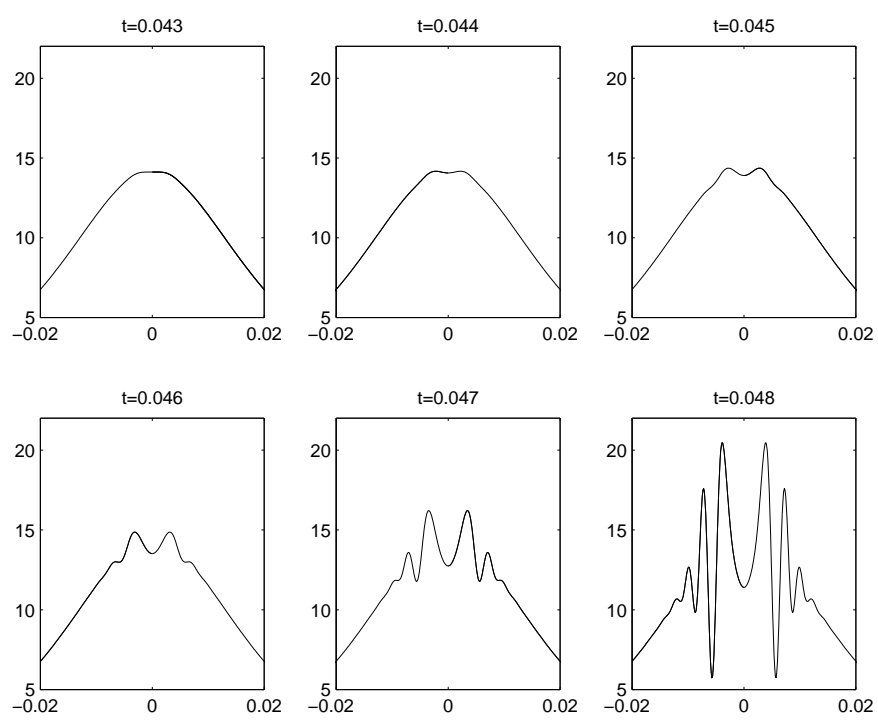

Figure 19: Tip curvature for $S=10^{-8}$ at early times near $t_{d}=0.0463$. Graph courtesy of Ceniceros \& Hou [35].
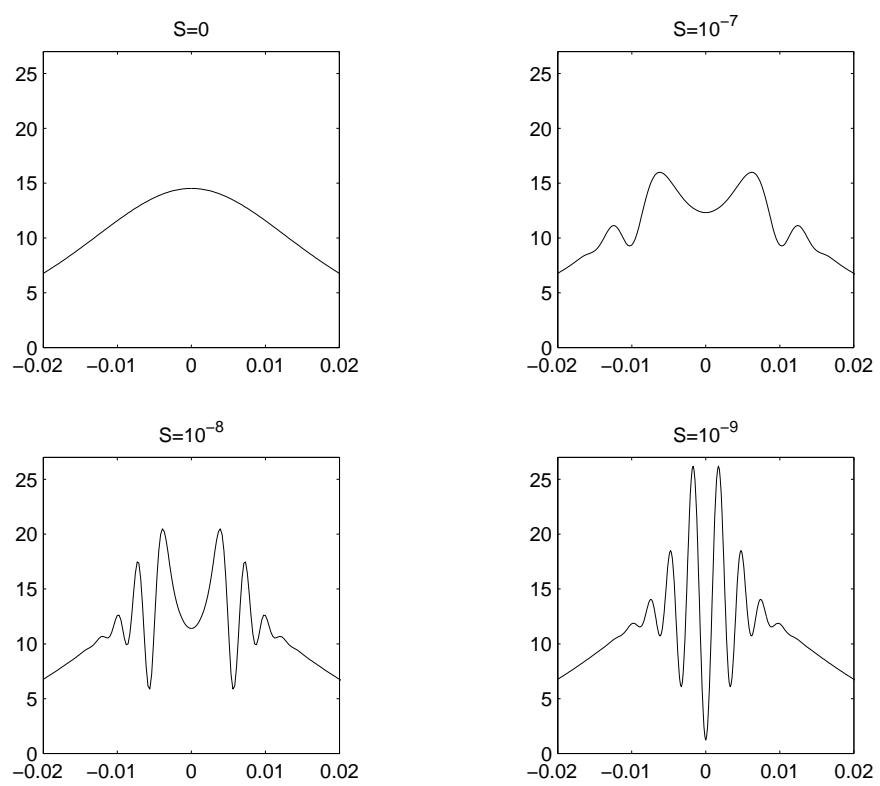

Figure 20: Curvature vs $\alpha / 2 \pi$ around one tip of the interface at $t=0.048$ for $S=0,10^{-7}, 10^{-8}$, and $10^{-9}$. $N=8192$. Precision level is 60 digits, with Filter level of $10^{-56}$. Graph courtesy of Ceniceros \& Hou [35]. 


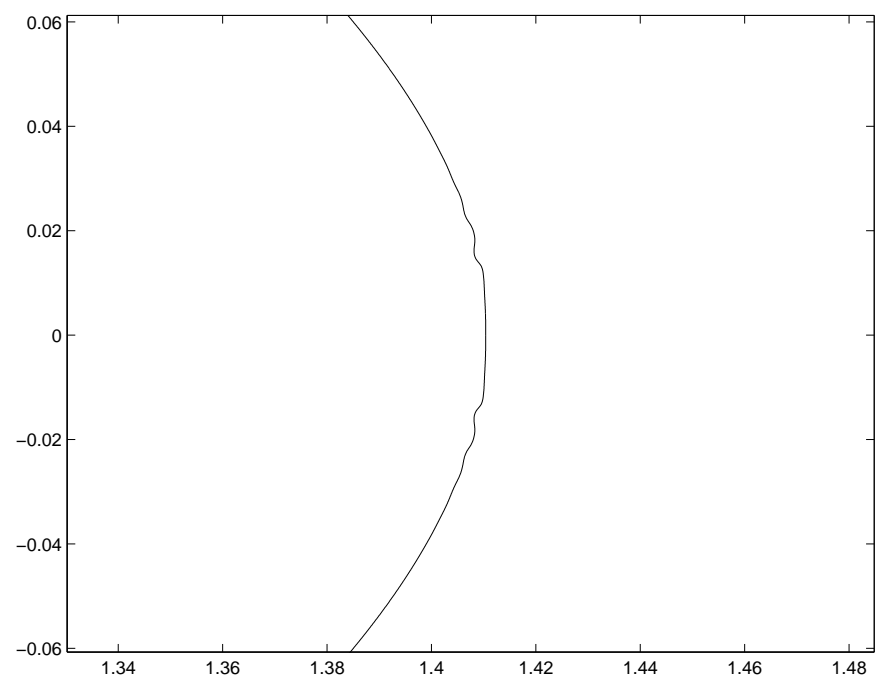

Figure 21: Close-up of the interface at $t=0.0502$ for $S=10^{-8}$. Graph courtesy of Ceniceros \& Hou [35].
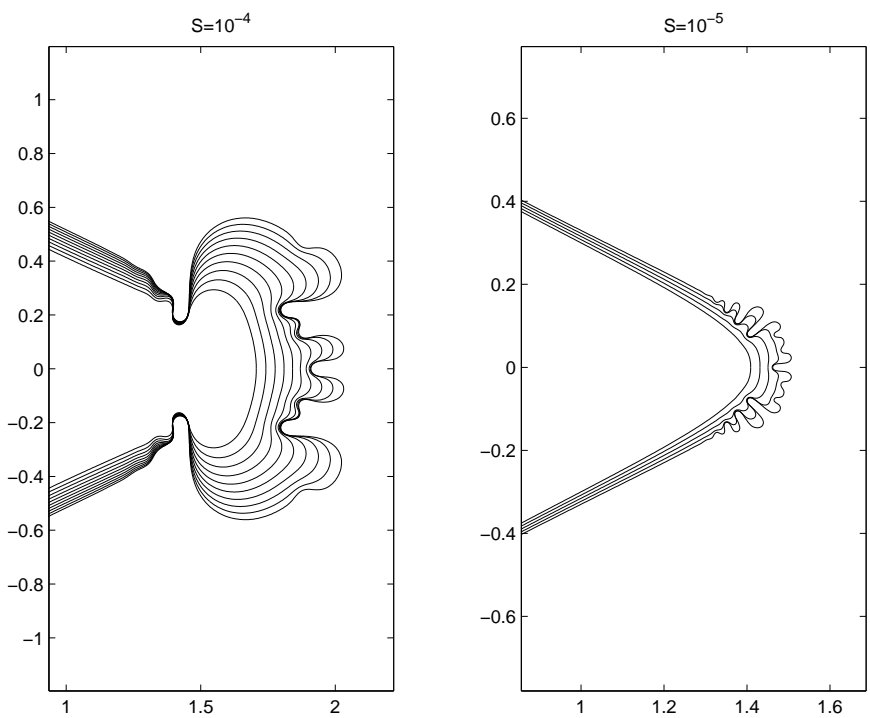

Figure 22: Close-ups of the Hele-Shaw interface at different times for $S=10^{-4}(t=0.23-0.50$, with a 0.03 time difference between profiles) and $S=10^{-5}(t=0.05-0.09$, with a 0.01 time difference between profiles). Computation performed with the equal-arclength method. ( $c f$. Fig. 11 of Siegel et al. [179].) Graph courtesy of Ceniceros \& Hou [35]. 


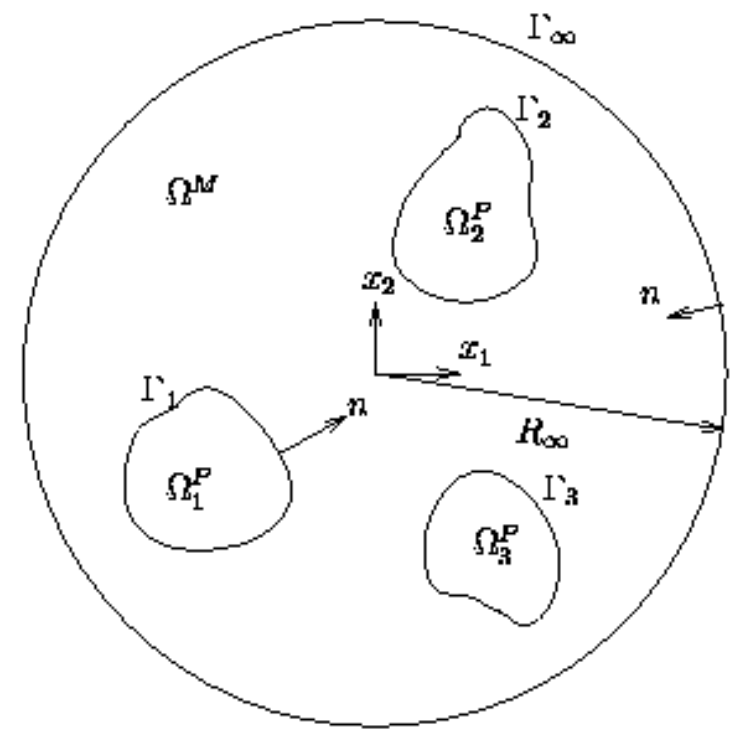

Figure 23: A two phase domain with three precipitates $(p=3)$.
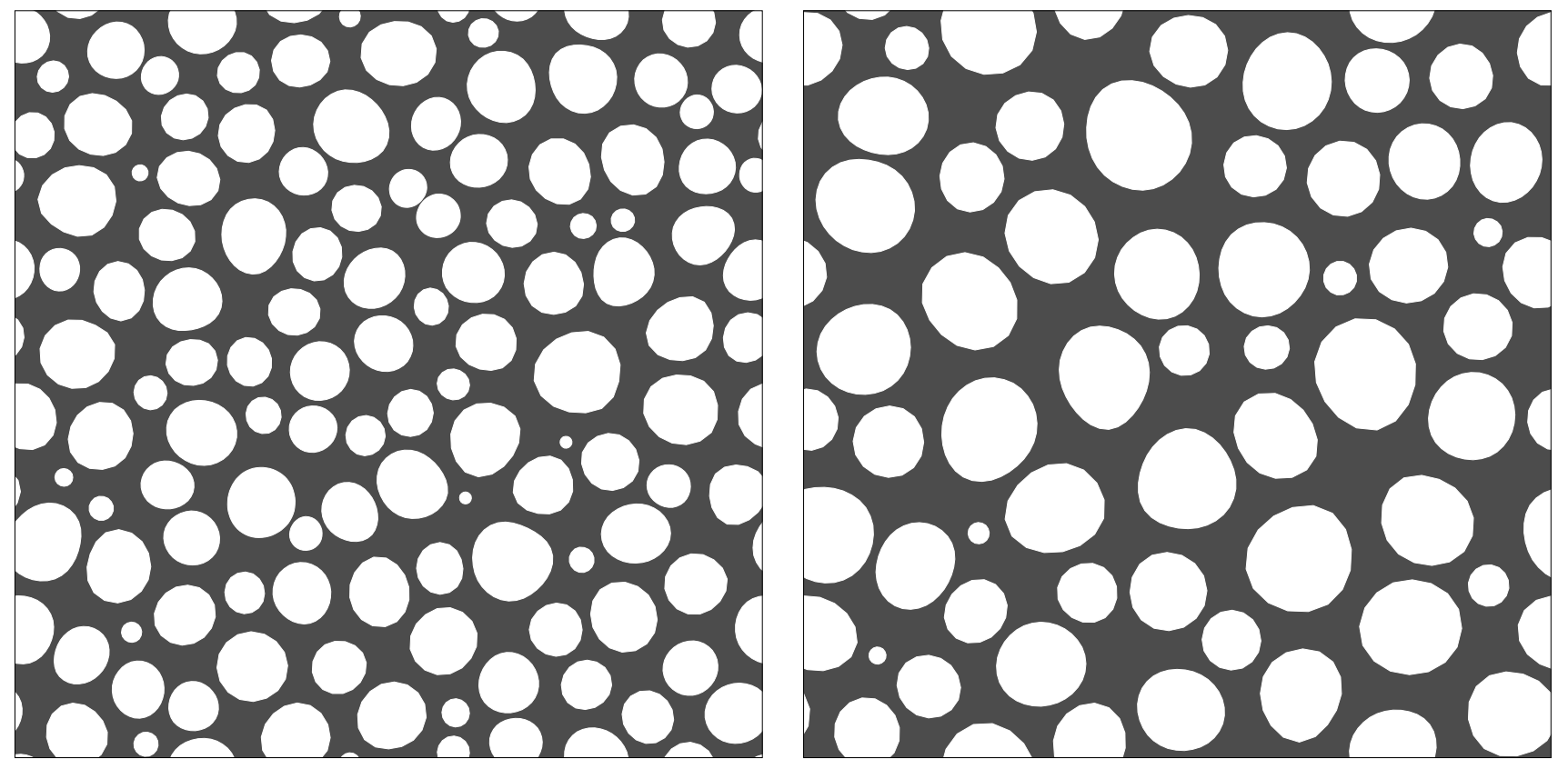

Figure 24: The late stages of diffusional coarsening with no elasticity $(Z=0)$. Left: moderate time; Right: late time. Graph courtesy of Akaiwa \& Meiron [1]. 

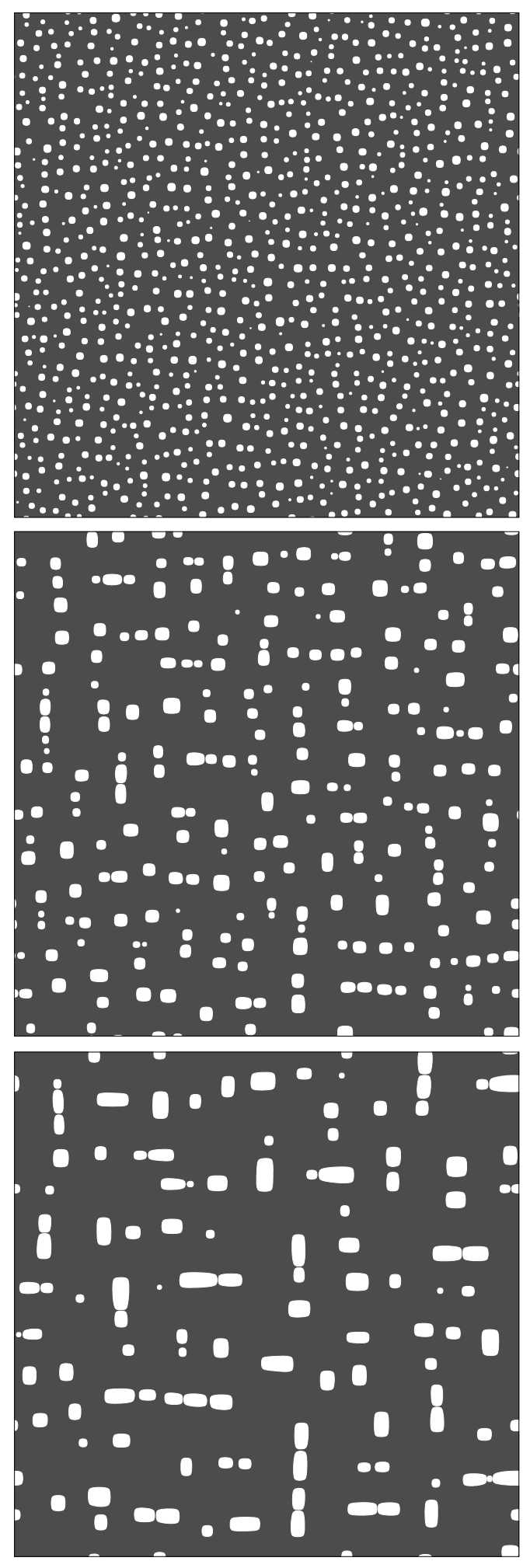

Figure 25: Coarsening in homogeneous, cubic elasticity. Upper graph: early time $(Z=2.0)$; Middle graph: moderate time $(Z=4.1)$; lower graph: late time $(Z=6.1)$. Graph courtesy of Thornton, Akaiwa \& Voorhees [190]. 


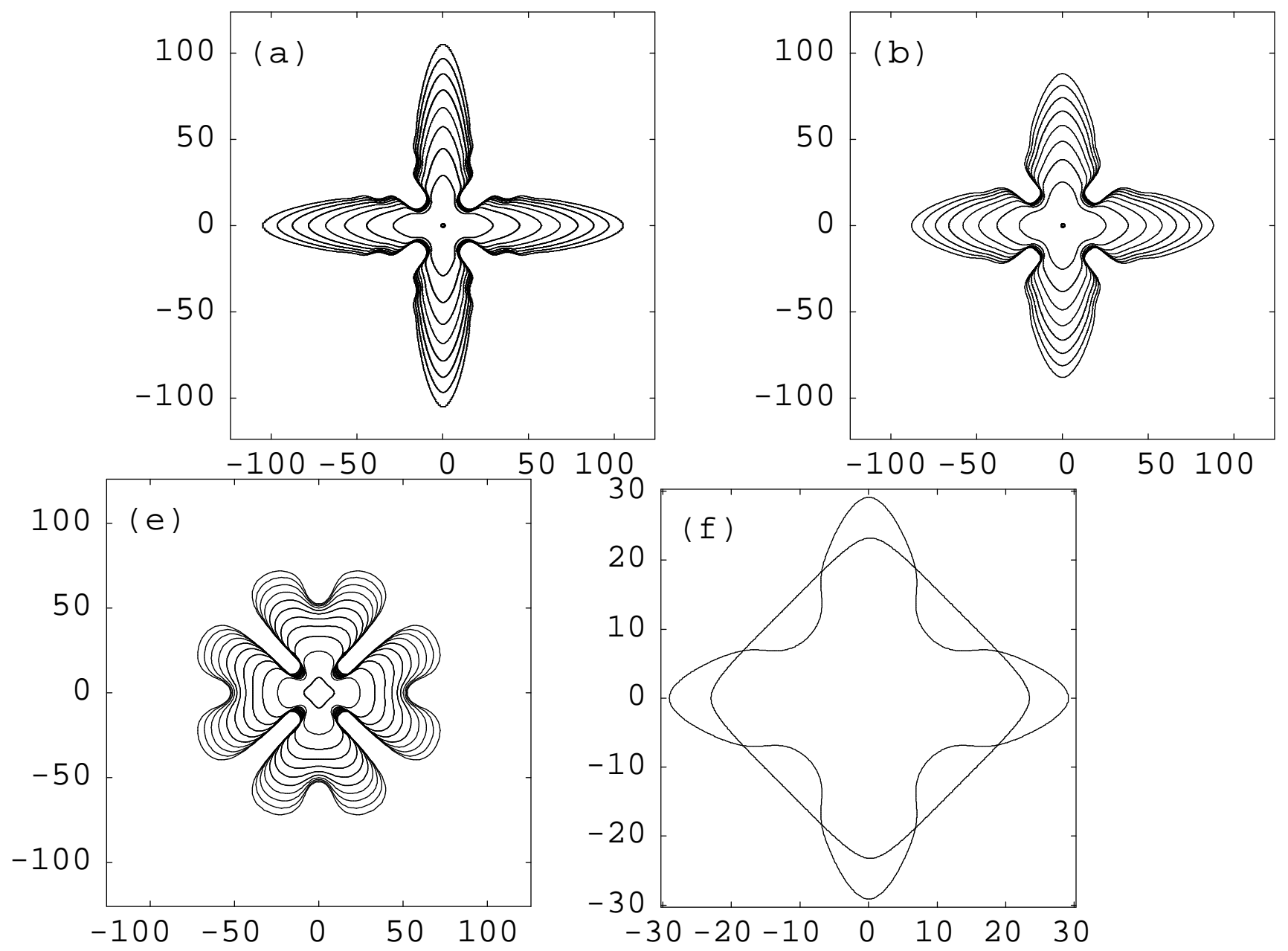

Figure 26: A series of different precipitate shapes under growth conditions in isotropic, inhomogeneous elastic media with shear misfit. In (a)-(c), the flux is $J=10$. In (a), the shear modulus of the precipitate is twice that of the matrix. In (b), the opposite case is shown. In (c), there is no elasticity $(Z=0)$. In (d), the non-convex shape is from (a) at time $t=20$ while the convex shape is the corresponding equilibrium shape. Graph courtesy of Jou, Leo \& Lowengrub [93]. 

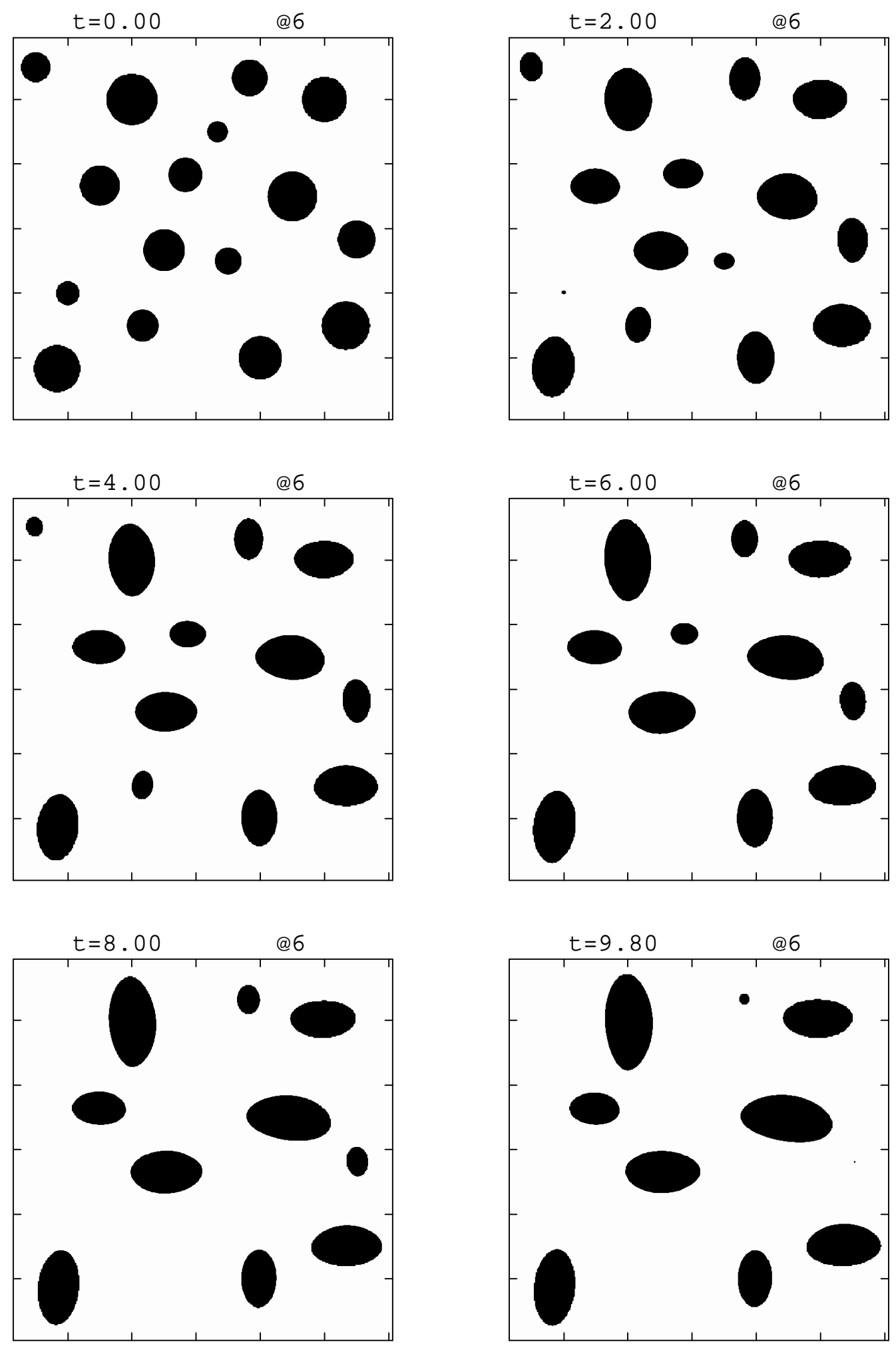

Figure 27: The coarsening of 16 precipitates in an isotropic, inhomogeneous elastic media. Graph courtesy of Jou, Leo \& Lowengrub [93]. 

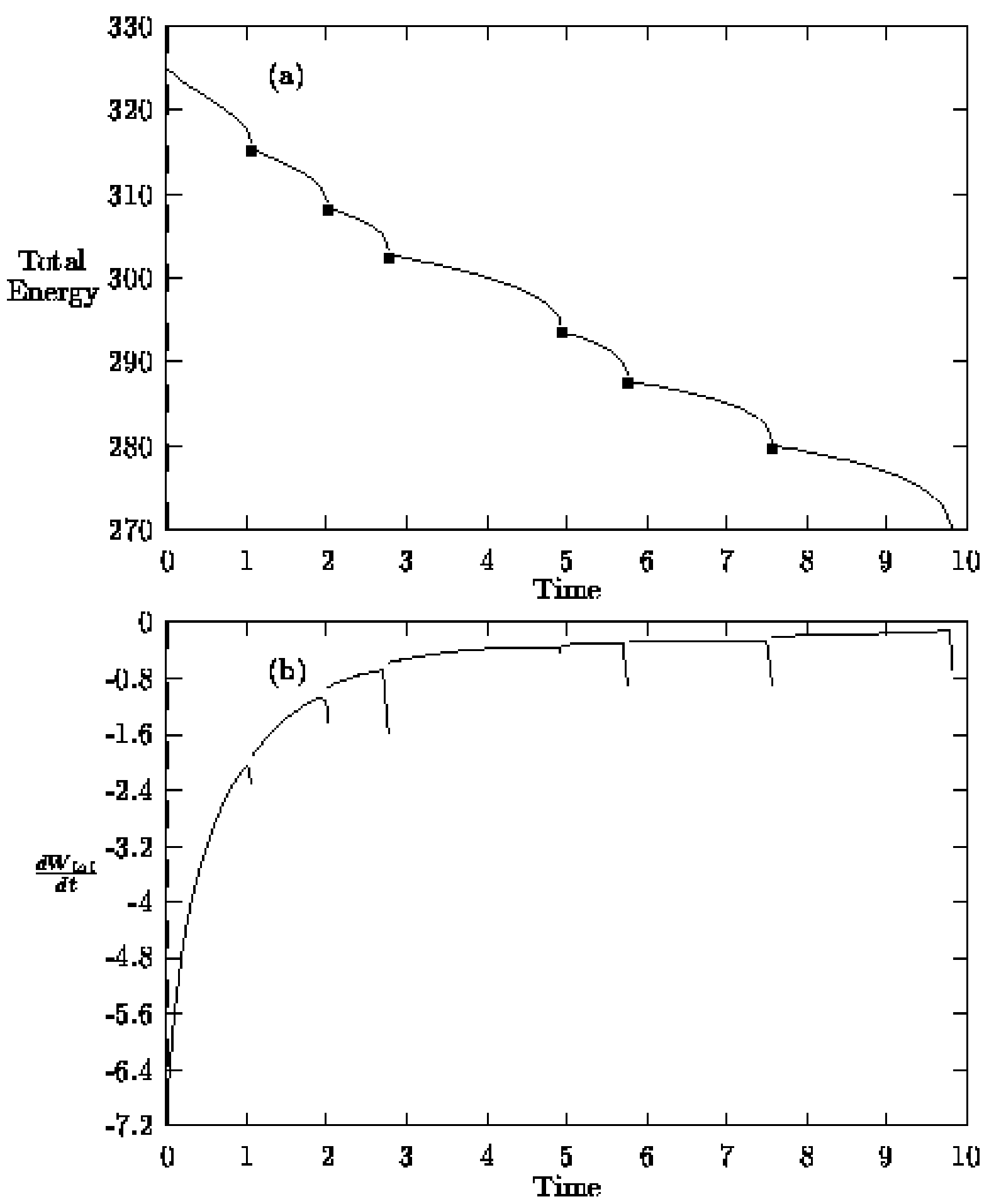

Figure 28: The behavior of the energy $W$ and $\dot{W}$ for the coarsening simulation shown in figure 27. The filled circles correspond to the times at which precipitates are removed. Graph courtesy of Jou, Leo \& Lowengrub [93]. 

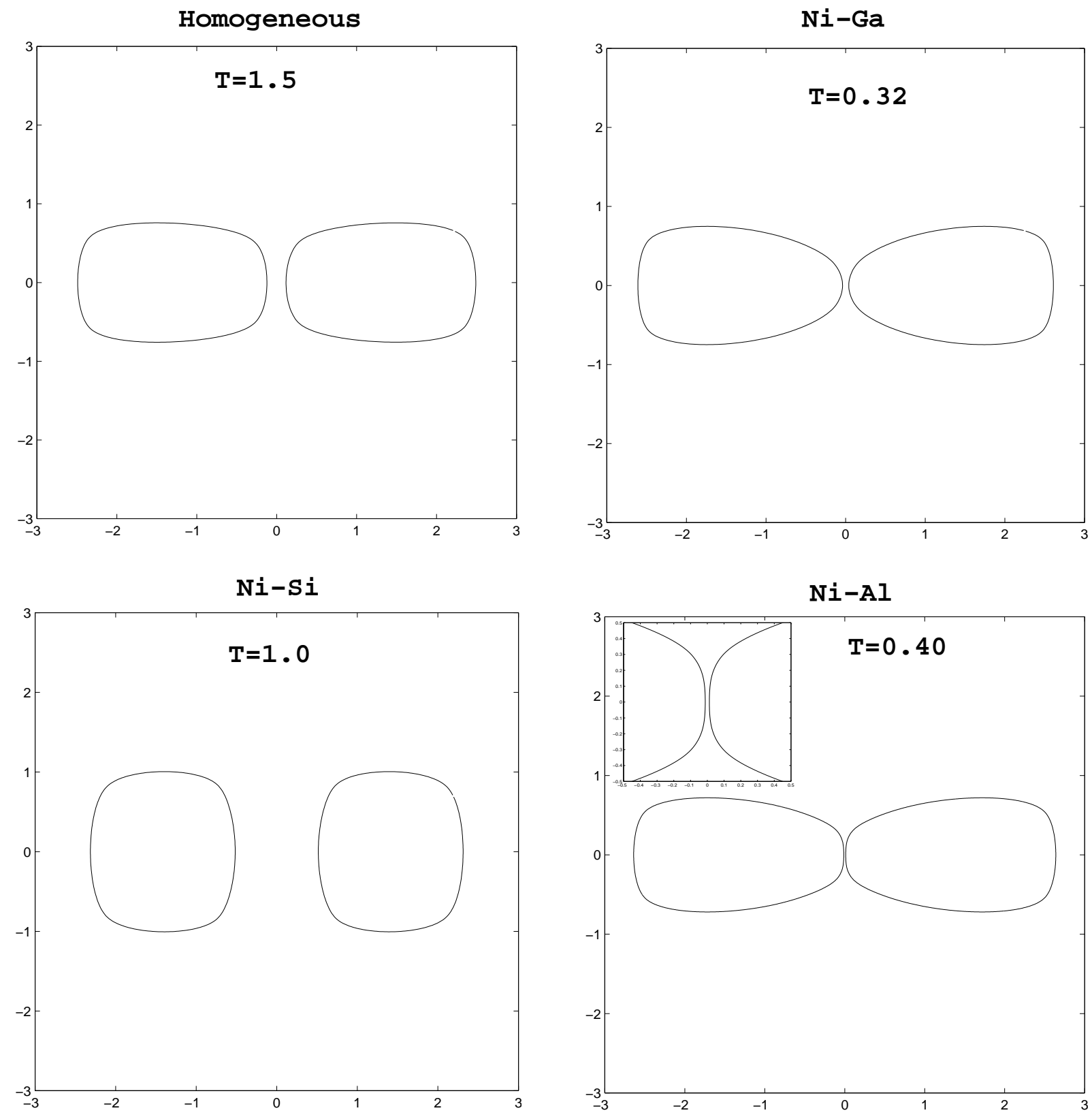

Figure 29: The behavior of two precipitates in inhomogeneous, cubic anisotropic elasticity. Graph courtesy of Leo, Lowengrub \& Nie [112]. 

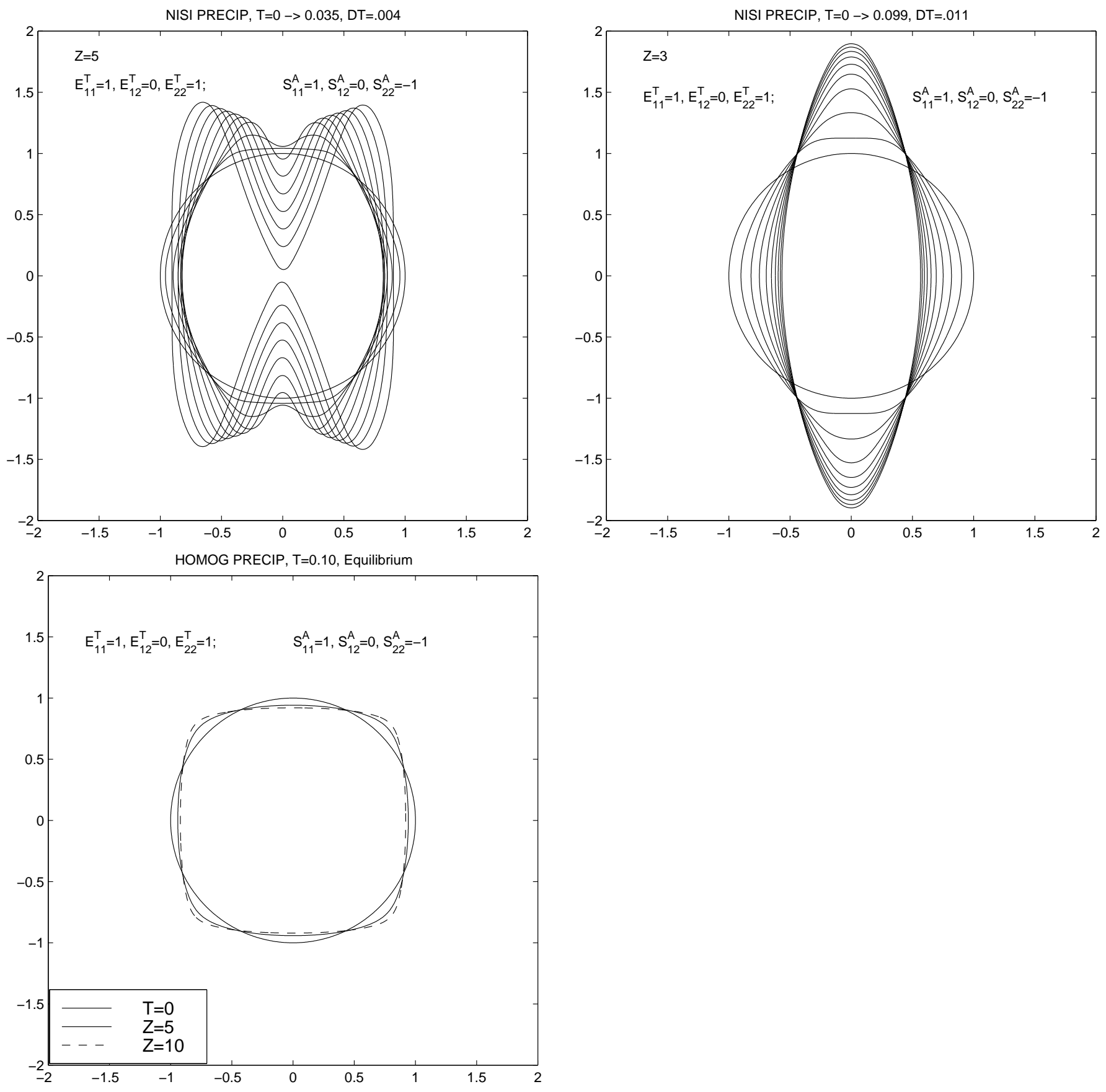

Figure 30: The elastic morphological instability of a misfiting single $N i_{3} S i$ precipitate in an applied shear. In (a), $Z=5$. In (b), $Z=3$. In (c), a homogeneous precipitate is shown for comparison with $Z=5$ and $Z=10$. Graph courtesy of Leo, Lowengrub \& Nie. 